\title{
Adenosine Receptor Oligomerization and G-protein Complex Stoichiometry Revealed by Electron Microscopy
}

\author{
Jennifer Noël Wingard \\ Gordonsville, VA \\ M.S. Microbiology \& Immunology, Loyola University of Chicago, 1998 \\ B.S. Biology, Randolph-Macon Woman's College, 1996

\begin{abstract}
A Dissertation Presented to the Graduate Faculty of the University of Virginia in Candidacy for the Degree of

Doctor of Philosophy

Interdisciplinary Program in Biophysics
\end{abstract} \\ University of Virginia \\ May, 2015
}


(C) 2015 Jennifer N. Wingard

All rights reserved 


\begin{abstract}
The $\mathrm{A}_{2 \mathrm{~A}}$ adenosine receptor $\left(\mathrm{A}_{2 \mathrm{~A}} \mathrm{AR}\right)$ belongs to a large superfamily of receptors (G-Protein Coupled Receptors), which are responsible for conveying signals from numerous external stimuli through their seven transmembrane helices to the membraneassociated heterotrimeric G-proteins. Drugs that target GPCRs comprise approximately $40 \%$ of the pharmaceutical market. Expressed highly in the brain, where it is thought to heterodimerize with the dopamine receptor, the $\mathrm{A}_{2 \mathrm{~A}} \mathrm{AR}$ is the target of drugs treating Parkinson's disease [1]. In the cardiovasculature, $\mathrm{A}_{2 \mathrm{~A}} \mathrm{AR}$ blockade yields protection from ischemia and hypoxia [2]. Additional studies have delineated the roles of $\mathrm{A}_{2 \mathrm{~A}} \mathrm{AR}$ in immunomodulation [2]. Detailing the $\mathrm{A}_{2 \mathrm{~A}} \mathrm{AR}$ molecular architecture with a thorough understanding of GPCR dimerization is crucial to understanding mechanisms of transmembrane signaling and the development of new therapeutics that target the dimer interface. The publication of the X-ray crystal structure and three-dimensional (3D) single particle electron microscopy $(\mathrm{EM})$ reconstructions of the $\beta_{2}$ adrenergic receptor $\left(\beta_{2} \mathrm{AR}\right)$ in a complex with Gs by 2012 Nobel prize-winner Dr. Brian Kobilka and colleagues provided surprising new insight into the flexibility of the helical domain of Gs and the direct mechanism of nucleotide exchange. Additional GPCR complex structures are necessary to corroborate this novel finding and determine whether the exchange mechanism is common to other G-proteins. Two-dimensional electron crystallography [3] and atomic force microscopy studies [4] suggest that the GPCR rhodopsin exists as a physiological dimer in native rod outer segment disc membranes; single particle 3D reconstructions of detergentsolubilized rhodopsin also suggest that the receptor is a dimer when associated with the G-
\end{abstract}


protein [5]. In contrast, 3D reconstructions of detergent-solubilized $\beta_{2} \mathrm{AR}-\mathrm{Gs}$ complexes suggest that the G-protein-bound receptor exists as a monomer [6, 7]. To date, rhodopsin is the only GPCR that has been crystallized within the milieu of the lipid bilayer. Unlike other Class A GPCRs, it is unusual in that it has a covalently bound ligand, 11-cis retinal. It remains unclear how GPCRs that bind diffusible ligands are organized within the lipid bilayer. To delineate the topology of the adenosine receptor within the lipid bilayer and with respect to the G-protein, my research has utilized electron microscopy and the complementary approaches of single particle image analysis and two-dimensional (2D) electron crystallography. The results provide insights about adenosine receptor-G-protein complex formation and suggest parallel dimerization of $\mathrm{A}_{2 \mathrm{~A}} \mathrm{AR}$ that is mediated by interactions between $\alpha$-helices $\mathrm{H} 1$ and $\mathrm{H} 8$ in the near-native environment of a lipid bilayer. 
For Emma Jane,

Dorothy \& Richard Wingard, in memoriam. 


\section{Epigraph}

An Ode to GPCRs

Seven passes through the membrane

And light becomes law

A feeling becomes transduced

Through an orbicular sea that floats between

Clouds of things like sphingomyelin

Sail mighty molecular machines

Parting the waves in rafts

Anchored in by oily tails

Rudders

Clasping

Energy transferring

To three-membered

Schools of fish entangled in their tentacles

Which in a flash diverge, and emit

Exploring the cytoplasmic ocean

To harness the energy

To turn the sun

Into a leap

Of science

In sight 


\section{Table of Contents}

Definitions

.11

\section{Chapter 1}

G-protein Coupled Receptors and the G-proteins ...............................15

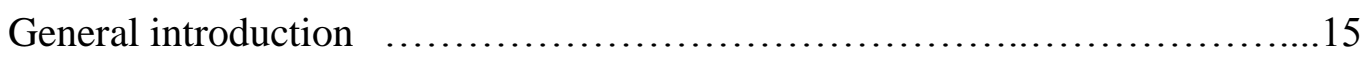

G-protein coupled receptors: background \& significance $\quad$.............................17

The adenosine receptors $\quad$...................................... 17

G-protein coupled receptor diversity ...................................................18

Adenosine receptors $\quad$........................................21

Relevance to human disease ............................22

Prominent structural features of G-protein coupled receptors $\quad$....23

Ligand binding pocket $\quad$...............................26

The E/DRY ionic Lock $\quad$.................................28

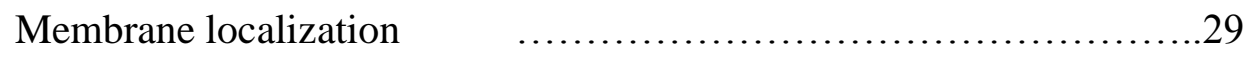

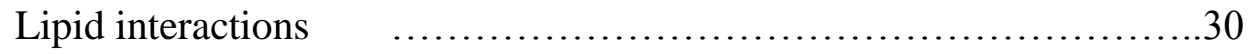

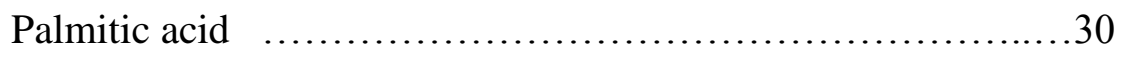

Cholesterol $\quad$................................................ 31

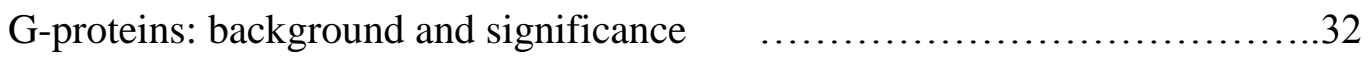

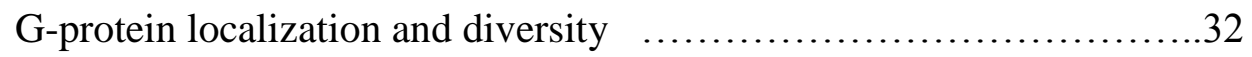

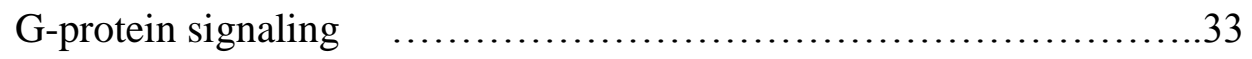

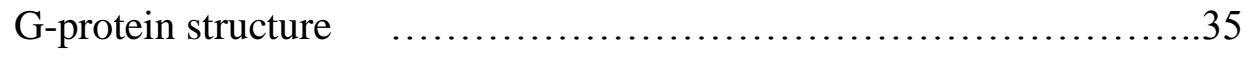


G-protein coupled receptor and G-protein interactions

\section{Chapter 2}

\section{Electron Microscopy and Single Particle Analysis of Adenosine}

Receptors and Receptor-G-protein Complexes $\quad$.................................54

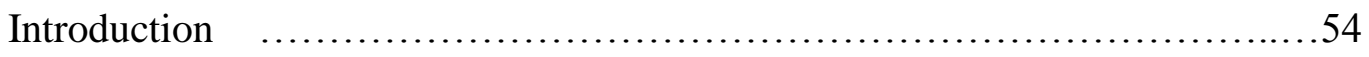

Insights from single particle analysis of other GPCRs $\quad \ldots \ldots \ldots \ldots . .56$

Results 


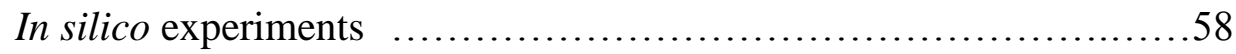

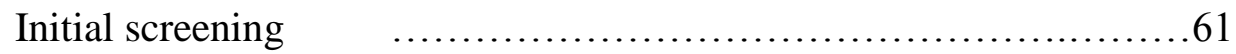

$3 \mathrm{D}$ reconstructions of adenosine receptor-G-protein complexes $\quad \ldots 61$

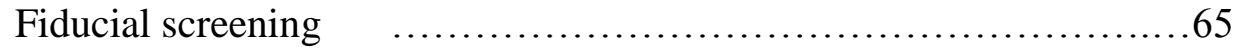

$3 \mathrm{D}$ reconstructions of $\mathrm{A}_{1}$ and $\mathrm{A}_{2 \mathrm{~A}}$ adenosine receptors $\quad \ldots \ldots \ldots .67$

Discussion $\quad$.................................................... 71

Experimental methods $\quad$....................................... 74

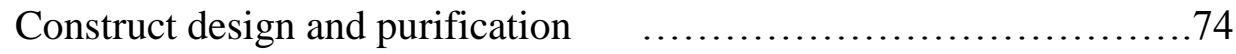

Sample preparation and electron microscopy .....................76

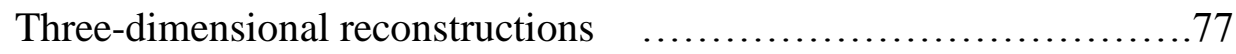

Fab preparation and nanogold labeling $\quad$.......................78

\section{Chapter 3}

\section{A Membrane-Associated A2A Adenosine Receptor Dimer Revealed} by Two-dimensional Electron Crystallography ...................................80

Abstract $\quad$........................................................ 80

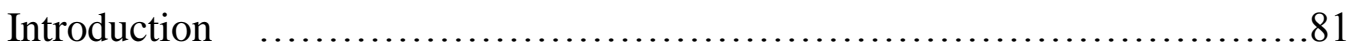

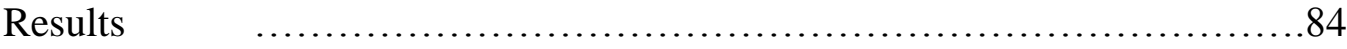

Discussion $\quad$..................................................... 91

Materials and Methods $\quad$................................................95

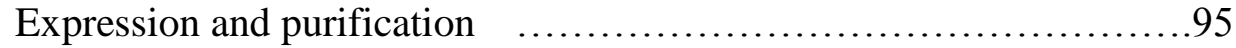

Two-dimensional crystallization trials $\quad$.....................96

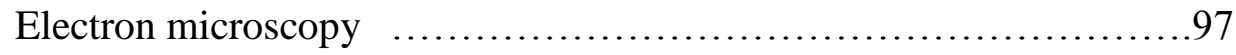

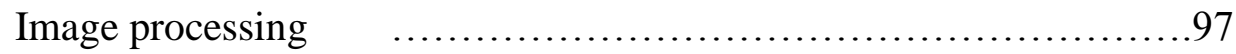




\section{Chapter 4}

Micro Electron Diffraction

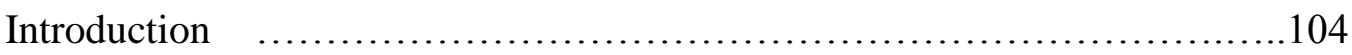

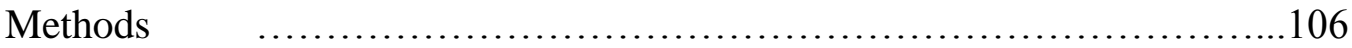

Solubilization, purification, and crystallization of $\mathrm{A}_{2 \mathrm{~A}} \mathrm{AR}$ in LCP 106

Cryopreservation of crystals grown by vapor diffusion $\quad$...........107

Cryopreservation of crystals grown in lipidic cubic phase $\quad$...........107

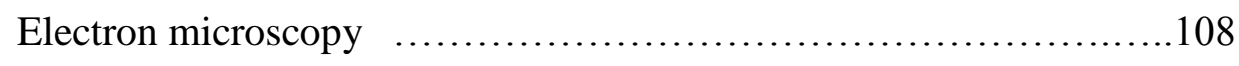

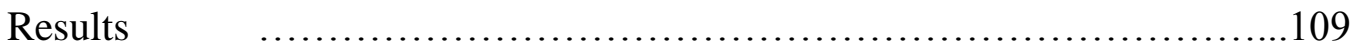

Diffraction of control crystals ......................................109

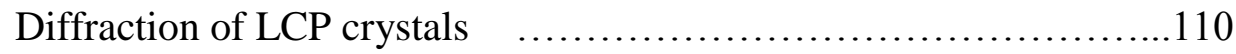

Diffraction of bicelle crystals ...................................112

Discussion $\quad$......................................................... 113

\section{Chapter 5}

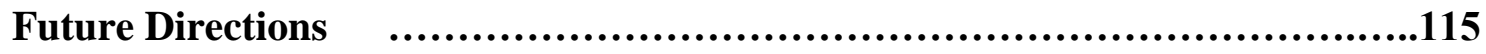

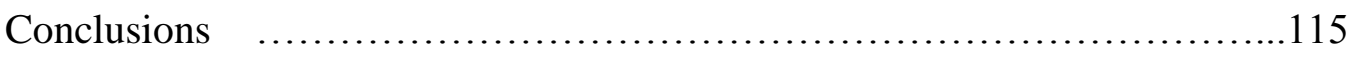

Methods development in electron microscopy $\quad$........................118

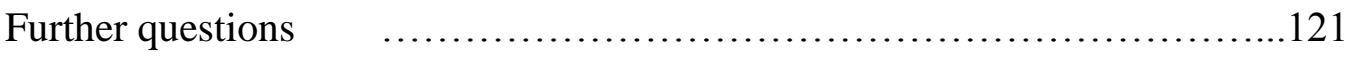

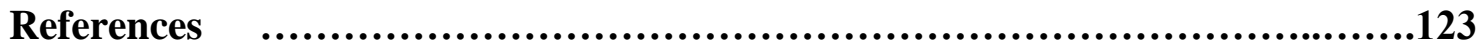

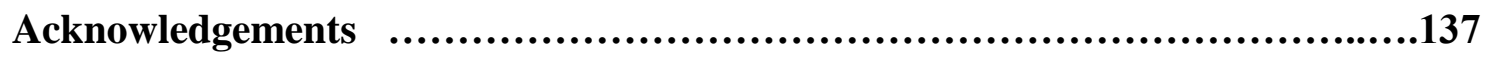




\section{Definitions}

2D (two-dimensional)

2Dxdb (two-dimensional crystallization database)

3D (three-dimensional)

$\mathrm{A}_{1} \mathrm{AR}\left(\mathrm{A}_{1}\right.$ adenosine receptor)

$\mathrm{A}_{2 \mathrm{~A}} \mathrm{AR}\left(\mathrm{A}_{2 \mathrm{~A}}\right.$ adenosine receptor)

$\mathrm{A}_{2 \mathrm{~B}} \mathrm{AR}\left(\mathrm{A}_{2 \mathrm{~B}}\right.$ adenosine receptor)

$\mathrm{A}_{3}\left(\mathrm{~A}_{3}\right.$ adenosine receptor)

AFM (Atomic force microscopy)

AIF4 (aluminum fluoride)

AMP (adenosine monophosphate)

AT1 (angiotensin receptor 1)

ATP (adenosine triphosphate)

$\beta_{1} \mathrm{AR}$ ( $\beta 1$ adrenergic receptor)

$\beta_{2} \mathrm{AR}$ ( $\beta 2$ adrenergic receptor)

BRET (bioluminescence energy transfer)

cAMP (cyclic adenosine monophosphate)

$\mathrm{CD}$ (cyclodextrin)

CGS-21680 (3-[4-[2-[ [6-amino-9-[(2R,3R,4S,5S)-5-(ethylcarbamoyl)-3,4-dihydroxy -oxolan-2-yl]purin-2-yl]amino]ethyl]phenyl]propanoic acid, an $\mathrm{A}_{2 \mathrm{~A}}$ agonist)

CHS (cholesterol hemisuccinate)

$\mathrm{CRF}_{1} \mathrm{R}$ (corticotropin releasing factor receptor type 1)

$\mathrm{Ct}$ (carboxyl terminus)

CTF (contrast transfer function) 
$\mathrm{D}_{1}$ (D1 dopamine receptor)

DAG (diacylglycerol)

ECL2 (extracellular loop 2)

ECL3 (extracellular loop 3)

EM (electron microscopy)

EPR (Electron paramagnetic resonance)

ERK (extracellular signal regulated kinase)

FRET (Förster resonance energy transfer)

$\mathrm{G}_{\mathrm{i} / \mathrm{o}}$ (inhibitory $\mathrm{G}$ protein)

$\mathrm{G}_{\mathrm{s}}$ (stimulatory $\mathrm{G}$ protein)

GABAB (gamma-aminobutyric acid receptor B)

GDP (guanosine diphosphate)

GPCR (G-protein coupled receptor)

GTP (guanosine-5'-triphosphate)

H1 (transmembrane $\alpha$-Helix I)

H4 (transmembrane $\alpha$-Helix IV)

H5 (transmembrane $\alpha$-Helix V)

H6 (transmembrane $\alpha$-Helix VI)

H8 (transmembrane $\alpha$-Helix VIII)

ICL3 (intracellular loop 3)

$\mathrm{IP}_{3}$ (inositol triphosphate)

LCP (lipidic cubic phase)

MD (molecular dynamics) 
mGluR (metabotropic glutamate receptor)

mGluR (metabotropic glutamate receptor)

microED (micro [crystal] electron diffraction)

NFkB (nuclear factor kappa- light- chain- enhancer of activated B cells)

NMR (nuclear magnetic resonance)

$\mathrm{Nt}$ (amino terminus)

$\mathrm{OPRM}_{1}$ (opioid receptor mu 1)

PC (phosphatidylcholine)

PE (phosphatidylethanolamine)

$\mathrm{PIP}_{2}$ (Phosphatidylinositol 4,5-bisphosphate)

POPC (1-palmitoyl-2-oleoyl-sn-glycero-3-phosphocholine)

POPE (1-palmitoyl-2-oleoyl-sn-glycero-3-phosphoethanolamine)

RGS4 (regulator of G-protein signaling 4)

Rho (rhodopsin)

SEC (size exclusion chromatography)

SMO (smoothened receptor)

T4L (T4 lysozyme)

TEM (transmission electron microscopy)

Three-dimensional (3D)

TM (transmembrane helix)

Two-dimensional (2D) 
UK-432097 (6-(2,2-diphenylethylamino)-9-((2R,3R,4S,5S)-5-(ethylcarbamoyl)-3,4dihydroxytetrahydrofuran-2-yl)- $N$-(2-(3-(1-(pyridin-2-yl)piperidin-4yl)ureido)ethyl)-9H-purine-2-carboxamide, a selective $\mathrm{A}_{2 \mathrm{~A}}$ agonist)

WD40 (tryptophan-aspartic acid 40 amino acid repeat)

XRD (X-ray diffraction)

ZM-241285 (4-(2-[7-amino-2-(2-furyl) [1,2,4]-triazolo[2,3-a][1,3,5]triazin- 5-yl amino]ethyl) phenol, an $\mathrm{A}_{2 \mathrm{~A}}$ antagonist) 


\section{Chapter 1}

\section{G-protein Coupled Receptors and the G-proteins}

\section{General Introduction}

Nearly one-thousand genes in the human genome encode seven-pass transmembrane proteins known as G-protein coupled receptors (GPCRs). GPCRs respond to numerous extracellular stimuli such as light, neurotransmitters, peptides, odorants, pheromones and other small molecules, transmitting a signal through the membrane to a diverse variety of heterotrimeric G-proteins. Conformational changes in the receptor are communicated to the G $\alpha$ subunit of the heterotrimer, prompting the exchange of GDP for GTP. The components of the G-protein, G $\beta \gamma$ and the now activated G $\alpha$, separate and go on to activate multiple downstream effectors, initiating diverse cellular responses. The parameters that modulate coupling to the various possible G-protein combinations is yet unknown; however, we now have a much clearer picture of the dynamics of the activation process during the initial steps of transmembrane signaling. The last five years have produced landmark publications in the field that have dramatically expanded our understanding of G-protein activation.

The structure of opsin, the ligand free form of rhodopsin, co-crystallized with the C-terminal peptide of $\mathrm{G} \alpha$ first provided confirmation of biochemical studies suggesting how the G-protein might dock to the receptor [8]. Although overlaying the structure of G $\alpha$ in the context of the heterotrimer produces steric clashes with the membrane, this structure offered the first glimpse of G-protein association with the receptor [8]. The first high- 
resolution crystal structure of the $\beta 2$ adrenergic receptor ( $\beta 2 \mathrm{AR})$, by Cherezov et al, revealed cholesterol and palmitic acid at the interface of a crystallographic parallel dimer [9]. The occurrence of a conserved cholesterol binding motif amongst GPCRs has been suggested $[10,11]$. Additional studies suggest that palmitoylation of Helix 8 (H8) may act in concert with cholesterol [12], aiding receptor membrane localization to lipid rafts and dimerization [13]. Whether palmitic acid and cholesterol are involved in docking remains to be determined, as well as the conformational dynamics that take place during the activation process.

Historically, multiple conflicting models existed for G-protein activation, based on crystal structures of G-protein heterotrimers [14] [15]. A solution for how receptor activation is communicated to $\mathrm{G} \alpha$ and activation of the G-protein takes place was inferred from the X-ray crystal structure of the $\beta_{2} \mathrm{AR}-\mathrm{Gs}$ complex [16], which revealed an open conformation of the nucleotide binding site, suggesting a direct release mechanism for nucleotide exchange. The dynamic nature of the $\alpha$-helical domain of Gs during activation further pursued by electron microscopy (EM) and single particle image analysis, which indicated mobility of the $\mathrm{G} \alpha_{\mathrm{H}}$ domain of $\mathrm{G} \alpha$ with respect to the G$\alpha_{G T P a s e}$ domain [7].

With the advent and expanded usage of lipidic cubic phase crystallization and protein engineering, in particular the replacement of intracellular loop 3 (ICL3) with T4 lysozyme (T4L), there has been an exponential growth in the number of GPCR structures produced over the last five years. Despite the structural advances in the field of GPCR research and elucidation of the G-protein activation mechanism, there is still much to be 
understood about the receptor-G-protein complex. While the activation mechanism is known, critical steps in the activation process remain to be elucidated, including docking, the dynamics of the receptor-G-protein interface during activation, the interaction of the receptor and G-protein within the lipid environment of the membrane bilayer, and the extent and function of homo- and hetero-oligomerization. A canonical understanding of these events with respect to multiple GPCRs will be necessary to fully understand the diversity of cellular responses mediated through this important signaling superfamily. Toward this understanding, this work examines the $A_{1}$ and $A_{2 A}$ adenosine receptors $\left(A_{1} A R\right.$ and $\mathrm{A}_{2 \mathrm{~A}} \mathrm{AR}$, respectively), using two-dimensional electron crystallography and single particle EM analysis. This chapter discusses in detail the background on which these studies were based and provides an overall context for the experimental results.

\section{G-Protein Coupled Receptors: Background and Significance}

\section{The Adenosine Receptors}

The adenosine receptor belongs to a large superfamily of receptors (GPCRs), which are responsible for conveying signals from numerous external stimuli through their seven transmembrane $\alpha$-helices to the membrane-associated heterotrimeric G-proteins. The Gproteins in turn modulate a number of downstream intracellular effectors, controlling signaling for a plethora of important biological responses. Drugs that target GPCRs comprise approximately $40 \%$ of the pharmaceutical market, which is not surprising, as adenosine receptors alone have been implicated in such diseases as systemic sclerosis [17], Huntington's [18], inflammatory bowel disease [19], renal fibrosis [20], and asthma [21] . 


\section{G-Protein Coupled Receptor Diversity}

GPCRs are divided into four main classes by sequence homology. Cursory examination of Figure 1.1 [22] conveys the remarkable diversity of the GPCR superfamily. An alternative classification system divides GPCRs into six classes by functional similarity and sequence homology: Rhodopsin-like (Class A), Secretin (Class B), Metabotropic Glutamate (Class C), Fungal Mating Pheromone (Class D), Cyclic AMP (Class E), and Frizzled/Smoothened (Class F); Classes D and E are restricted to fungi and slime molds respectively [23]. Adenosine receptors belong to Class A, the largest and most widely studied due to the medical significance of its members. Members of Class A bind diverse ligands that include peptides, hormones and small molecules. Until 2007, just one GPCR structure was available, that of rhodopsin, a rather unusual GPCR family member as it is covalently bound to its ligand, 11-cis-retinal [24]. The relatively scant amount of structural data, considering this large and medically important class of molecules, is surprising unless one considers a number of complicating factors: the large hydrophobic surface area, that interaction of the receptor with membrane lipids is not easily accommodated by the largely hydrophilic structural approaches, the presence of conformationally-flexible loop regions, and the relatively large size of the receptor. Fortunately, advancements in membrane protein crystallization like the development of lipidic cubic phase and sponge phase crystallization, and particularly protein engineering, have facilitated the structural biology of GPCRs. In the most successful crystallization construct, the flexible third intracellular loop (ICL3) has been replaced with T4 lysozyme (T4L) [25], providing additional stability and crystal contacts. There are now crystal structures available for GPCRs in Classes A, B, $\mathrm{C}$, and $\mathrm{F}[26]$. A comparison of these structures reveals diversity in the location, size, and 


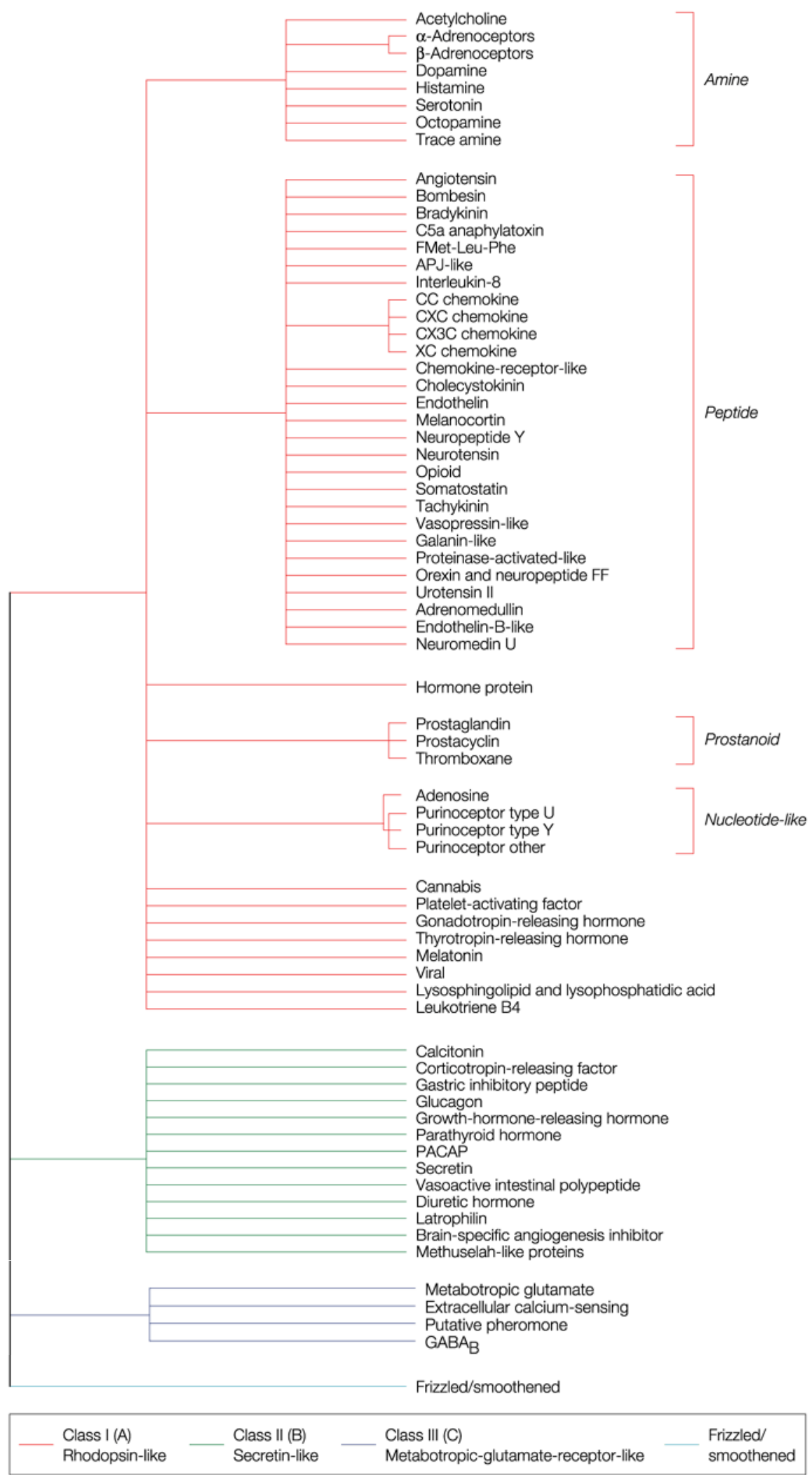

Figure 1.1. Classification and Diversity of G-Protein Coupled Receptors. Reprinted by permission from Macmillan Publishers Ltd: Nature Reviews Drug Discovery, Chalmers, D.T. and D.P. Behan, The use of constitutively active GPCRs in drug discovery and functional genomics, copyright 2002. 
morphology of the ligand binding pocket, as seen in Figure 1.2 [26]. After rhodopsin, the next crystal structures of GPCRs were those of the $\beta 1$ [27] and $\beta 2$ adrenergic receptors [9, 28]. These structures provided great insights into receptor activation. Relative to the antagonist-bound structure of the $\beta_{1} \mathrm{AR}$, the agonist-bound structure revealed a more compact binding pocket due to an inward tilt of transmembrane $\alpha$-Helix 5 (H5) [29]. Prior to the publication of the next GPCR structure, that of the $\mathrm{A}_{2 \mathrm{~A}} \mathrm{AR}$, a molecular dynamics (MD) docking competition, GPCR Dock 2008, challenged the MD community to predict the structure of $\mathrm{A}_{2 \mathrm{~A}} \mathrm{AR}-\mathrm{ZM} 241285$ [30]. The docking competition proved to be difficult, as the $\mathrm{A}_{2 \mathrm{~A}} \mathrm{AR}$ binding pocket was significantly different than either that of rhodopsin or the adrenergic receptors. The subsequently published crystal structure of $\mathrm{A}_{2 \mathrm{~A}} \mathrm{AR}-$ ZM241285 revealed unexpected differences in the location and orientation of the binding pocket as well as in the positions of extracellular loops [31], demonstrating that important variances exist even within the same class (e.g. rhodopsin and the $\mathrm{A}_{2 \mathrm{~A}} \mathrm{AR}$ ).
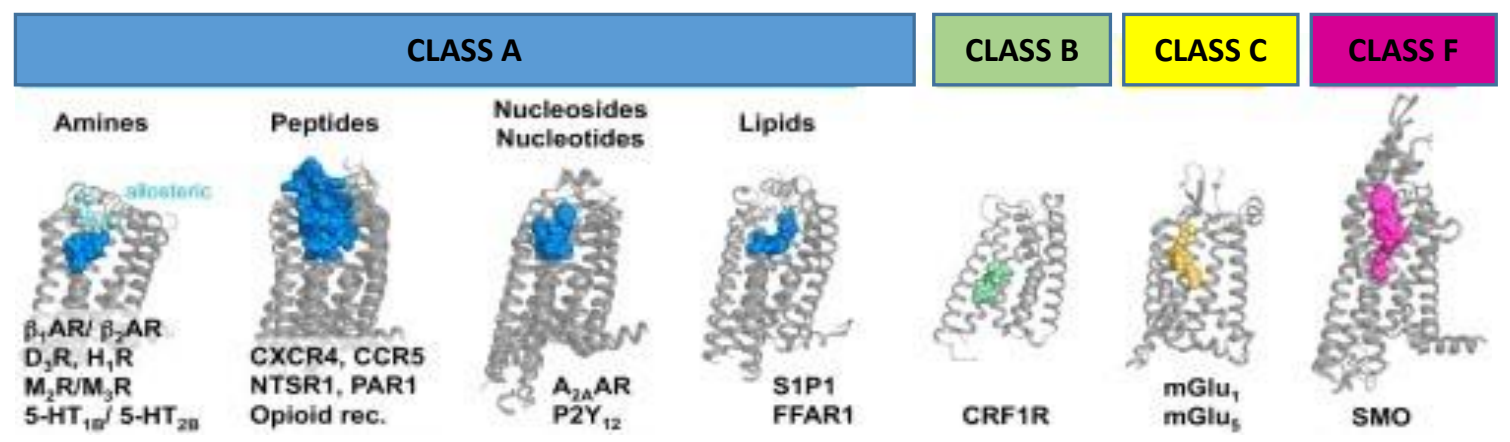

Figure 1.2. Ligand Pocket Diversity in Four Major GPCR Classes. Shonberg, J., et al., GPCR crystal structures: Medicinal chemistry in the pocket. Bioorg Med Chem, copyright 2014, reprinted with permission from Elsevier.

The structure of the first Class B receptor, corticotrophin-releasing factor receptor type $1\left(\mathrm{CRF}_{1} \mathrm{R}\right)$, revealed even more striking differences; relative to Class A GPCRs, its deep hydrophobic binding pocket deviates by $13-23 \AA$ in location from those in Class A 
[32]. More insight into ligand selectivity was revealed by the transmembrane structure of the first Class C GPCR, that of the glutamate receptor, mGluR5. A $6 \AA$ inward tilt of H5 on the extracellular face creates a very narrow opening to the allosteric binding pocket containing a coordinated water molecule in an extensive H-bonding network [33]. For Class F GPCRs, the crystal structure of the antagonist-bound Smoothened (SMO) receptor revealed a similarly narrow binding pocket, but a dramatically different extracellular face comprised of extended loops and an extended transmembrane $\alpha$-helix H6 due to the lack of conserved proline residues present in Class A receptors; primary ligand interactions in SMO are made with extracellular loops 2 and 3 and several conserved water molecules [34]. The structural variability observed between the classes has revealed determinants for ligand binding and informs the development of new therapeutics. Two classes of GPCRs remain without representative structures. While these GPCRs are not found in humans, structural analysis may hold promise for the development of anti-fungal agents and a deeper understanding of the evolutionary history of this diverse family of receptors.

\section{Adenosine Receptors}

There are four adenosine receptors expressed in humans, $\mathrm{A}_{1}, \mathrm{~A}_{2 \mathrm{~A}}, \mathrm{~A}_{2 \mathrm{~B}}$ and $\mathrm{A}_{3}$, all of which modulate important physiological responses via G-protein coupling. The receptors utilize different signaling pathways via selective coupling to $\mathrm{G} \alpha$ isoforms; $\mathrm{A}_{1} \mathrm{AR}$ decreases cAMP levels via binding to the inhibitor $G$ protein $\left(G_{i / o}\right)$, whereas the $A_{2 A} R$ stimulates adenylate cyclase activity through the stimulatory $G$ protein $\left(G_{s}\right)$. Within the human adenosine receptors, there is a high level of sequence conservation: $59 \%$ between the $A_{2 A}$ and $A_{2 B}$ receptors, and $49 \%$ between the $A_{1}$ and $A_{3}$ receptors [35]. Expression 
levels are variable and are based on tissue localization and extent of cellular stress. The endogenous agonist, adenosine, is generated as a result of cell damage, or via nucleotidasemediated hydrolysis of extracellular ATP. Other naturally occurring ligands include methyl xanthines such as caffeine, theophylline, and theobromine and the citrus-derived terpene, limonene [36].

\section{Relevance to Human Disease}

Adenosine receptors, particularly $\mathrm{A}_{2 \mathrm{~A}} \mathrm{AR}$, are concentrated in the smooth muscle of the coronary arteries, where they play a role in ischemic conditioning [2], expressed in immune cells such as mastocytes [37], macrophages [38], neutrophils [39], lymphocytes [40], and monocytes where they play roles in modulating inflammation [2], and in the basal ganglia, where they act to control motor function [1]. Their most critical role is in responding to hypoxic conditions. The $\mathrm{A}_{1} \mathrm{AR}$ and $\mathrm{A}_{2 \mathrm{~B}} \mathrm{AR}$ are down-regulated in response to hypoxic conditions to protect from a toxic accumulation of adenosine during cardiogenesis in chick embryogenesis; however, $\mathrm{A}_{2 \mathrm{~A}}$ and $\mathrm{A}_{3}$ adenosine receptors are not [41]. In the ischemic preeclamptic placenta, $\mathrm{A}_{2 \mathrm{~A}} \mathrm{AR}$ expression is upregulated [42]. During ischemic injury leading to renal fibrosis, there is also up-regulation of the $A_{2 B} A R$ and adenosine-mediated expression of fibrotic markers [43]. Differences in response may be attributable to differential expression based on tissue localization. While both $\mathrm{A}_{2 \mathrm{~A}} \mathrm{AR}$ and $\mathrm{A}_{2 \mathrm{~B}} \mathrm{AR}$ are commonly both expressed in the same cell type, one of the two is functionally predominant [44]. In a mouse model of chronic kidney disease, adenosine blockade protects against renal damage [20], suggesting the $A_{2 A} A R$ is involved in the pathology of the disease. $\mathrm{A}_{2 \mathrm{~A}} \mathrm{AR}$ up-regulation has also been observed in Parkinson's patients with 
dyskinesia [45] and in retinal ischemia [46]. Up-regulation of the $\mathrm{A}_{2 \mathrm{~A}} \mathrm{AR}$ in response to ischemic injury is thus common across multiple organ systems. The endogenous agonist, adenosine, is released during periods of cellular stress, arising from increased oxygen consumption [47] and cellular workload, excitatory neurotransmitter release [48], or cell membrane damage. The mechanism for the up-regulation of the $\mathrm{A}_{2 \mathrm{~A}} \mathrm{AR}$ during ischemic injury is not well understood; however, it is likely mediated by the transcription factor $\mathrm{NF \kappa B}$ [49]. Under conditions of oxidative stress, $\mathrm{A}_{1} \mathrm{AR}$ activation is protective against cardiac injury [50]. Hypoxia causes increased oxidative stress [51], which has been shown to upregulate $\mathrm{A}_{1} \mathrm{AR}$ expression in smooth muscle myocytes [52]. Similarly, the $\mathrm{A}_{2 \mathrm{~A}} \mathrm{AR}$ agonist CGS-21680 attenuated ischemia-induced apoptosis of the liver, which appears mediated by inhibition of interferon- $\gamma$ release [53]. Adenosine receptor blockade has been shown in many cases to be effective in ameliorating the effects of up-regulation as well as the effects of preconditioning hypoxia [54]; however, a more thorough understanding of adenosine receptor signaling is necessary for improved targeting in the disease state.

\section{Prominent Structural Features of G-Protein Coupled Receptors}

As mentioned previously, GPCRs are 7-pass transmembrane receptors, with three extracellular and three intracellular loops as shown in the schematic in Figure 1.3. Examining this schematic, it is apparent how ligand binding is coupled to receptor activation. Class A GPCRs have a short $\mathrm{N}$-terminus and an extended $\mathrm{C}$-terminal tail that forms a conserved amphipathic $\alpha$-helix denoted H8, which lays parallel to the membrane. H8 may be lipid modified, and in the Class C mGluR2 receptor, is conformationally 
sensitive to and stabilized by cholesterol [55]. $\mathrm{H} 8$ of $\mathrm{A}_{1} \mathrm{AR}$ and $\mathrm{A}_{2 \mathrm{~A}} \mathrm{AR}$, as well as many other Class A GPCRs, may also be phosphorylated following activation, which can modulate binding to other signaling partners such as adenylate cyclase [56] [57] and $\beta$ arrestins [58] as well as promote receptor internalization. In rhodopsin, phosphorylation of the C-terminus is inhibitory to high-affinity arrestin binding [59]. G-protein binding determinants will be discussed in the following section.

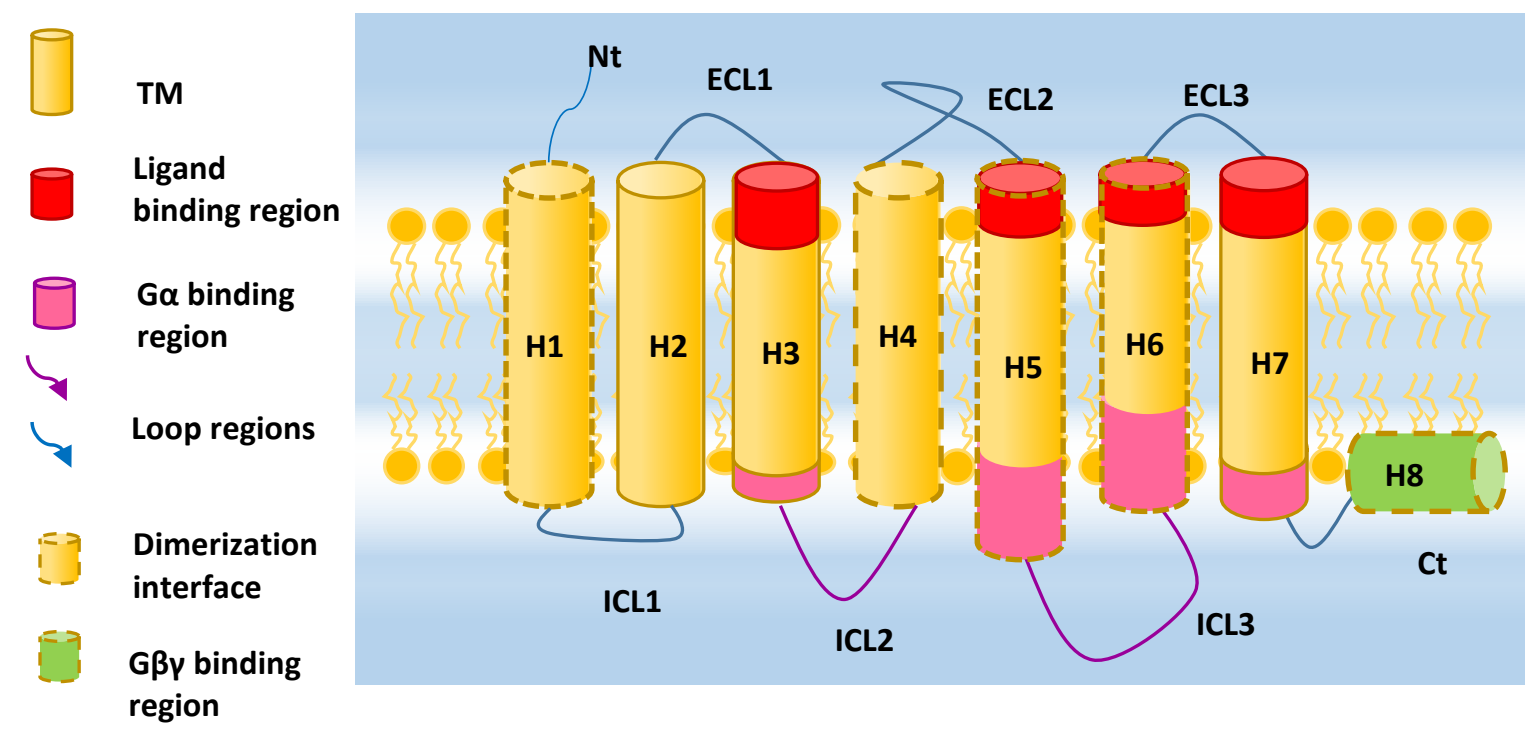

Figure 1.3. GPCRs are 7-Pass Transmembrane Receptors. ECL, extracellular loop. ICL, intracellular loop. H1, Helix 1. Nt, N-terminus. Ct, C-terminus. Regions implicated in ligand

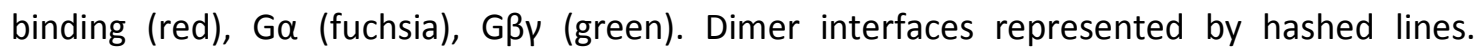
Observed interfaces include $\mathrm{H} 8-\mathrm{H} 1, \mathrm{H} 4-\mathrm{H} 5$, and $\mathrm{H} 6-\mathrm{H} 6$.

Understandably, sequence conservation between GPCRs is the greatest for the regions that encode the transmembrane (TM) $\alpha$-helices [60]; thus helical bundle orientation and packing are highly similar. The first five GPCR structures solved were rhodopsin, $\beta_{1} \mathrm{AR}, \beta_{2} \mathrm{AR}, \mathrm{A}_{2 \mathrm{~A}} \mathrm{AR}$ and opsin, which have a common core comprised of 97 residues with an average C $\alpha$ RMSD of $1.3 \AA$ [60]. Non-core residues have a high degree of structural 


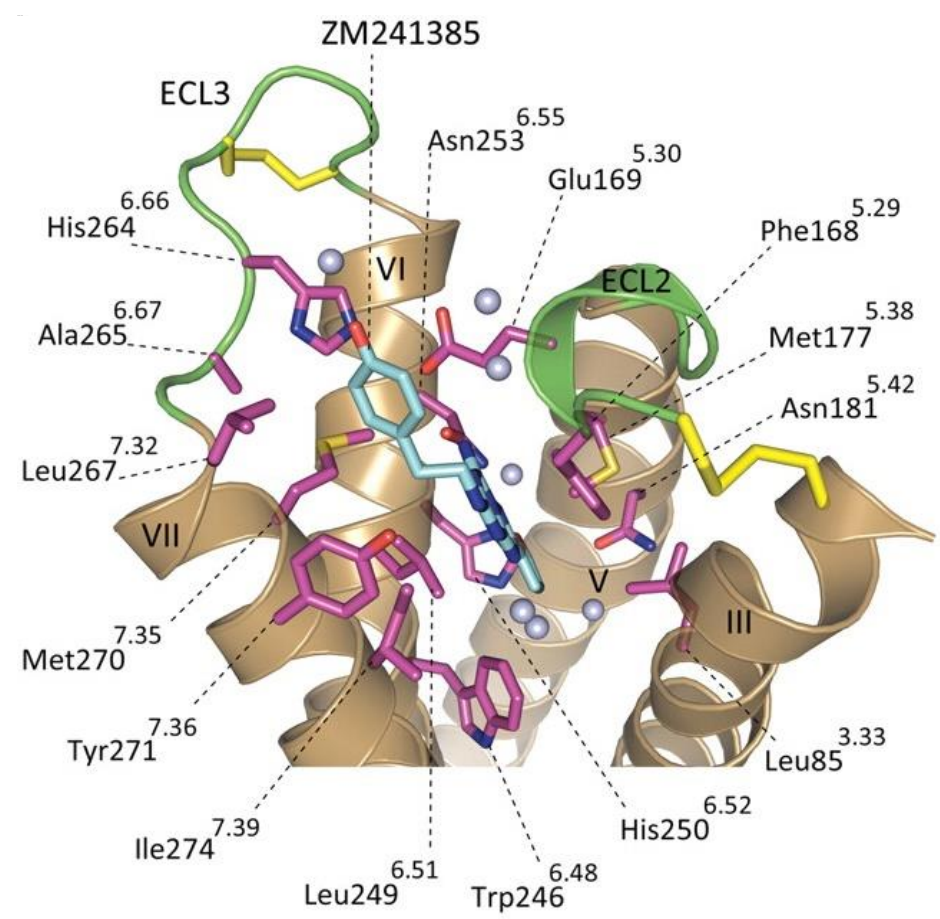

Figure 1.4. The $A_{2 A} A R$ Ligand Binding Pocket with Bound Antagonist ZM241385. From Jaakola, V.P., et al., The 2.6 angstrom crystal structure of a human $A_{2 A}$ adenosine receptor bound to an antagonist. Science, 2008. 322(5905): p. 1211-7. Reprinted with permission from AAAS.

divergence, likely due to differing binding and signaling specificities [60]. The binding pockets of the $\beta_{2} \mathrm{AR}$ and the $\mathrm{A}_{2 \mathrm{~A}} \mathrm{AR}$ are significantly different from that of rhodopsin, likely due to their binding diffusible ligands. The $\mathrm{A}_{2 \mathrm{~A}} \mathrm{AR}$ structure also included a feature not predicted by secondary structure prediction algorithms based on rhodopsin, a small helix within ECL2 [61], as seen in Figure 1.4. The structure of $A_{2 A} A R$ with a subtype selective antagonist, ZM241385, also revealed differences in the binding pocket with respect to the $\beta_{2} \mathrm{AR}$ and opsin [61]. Figure 1.2 provides a visual comparison of the ligand binding pocket with respect to the $\beta_{2} \mathrm{AR}$ and the $\mathrm{A}_{2 \mathrm{~A}} \mathrm{AR}$. 


\section{Ligand Binding Pocket}

As previously mentioned, unlike both rhodopsin and the $\beta_{2} \mathrm{AR}$, the ECL2 of $\mathrm{A}_{2 \mathrm{~A}} \mathrm{AR}$ is relatively disordered and extends away from the highly solvent-accessible binding pocket (Figure 1.4) [31]. The ECL2 of $\mathrm{A}_{2 \mathrm{~A}} \mathrm{AR}$ is a spatially-constrained random coil that makes three disulfide bonds with ECL1, two of which are unique to the $\mathrm{A}_{2 \mathrm{~A}} \mathrm{AR}$ [31]; a fourth disulfide bond in ECL3 creates a kink that constrains it, such that His264 is oriented into the binding site. Ultimately, this forms a rather rigid, open structure in which the binding cavity is exposed. The disulfides are critical, as mutation of the two cysteines in $\mathrm{A}_{1} \mathrm{AR}$ cause a loss of antagonist binding [31]. The disulfide maintains the architecture of the pocket through association of ECL2 and H3 [62]. In addition, the loop structure orients two Cys-adjacent residues, Phe168 and Glu169, which are critical residues for ligand binding. Electron density for Gln148 to Ser156 is missing in the $\mathrm{A}_{2 \mathrm{~A} A R}$ structure [31], and it is unclear what role, if any, that these residues may play in ligand binding. The antagonist that was visualized in the $\mathrm{A}_{2 \mathrm{~A}} \mathrm{AR}$ structure, ZM241385, contains a furan ring, which is located $\sim 3 \AA$ away from the conserved Trp246 "toggle switch" that is important in receptor activation [31]. This H6 tryptophan, common to Class A GPCRs, represented in the Figure 1.5 schematic, is understood to modulate the equilibrium between the inactive and active state [31]. On the basis of the position of retinal in the rhodopsin structure, it was proposed that the hydrophobic interactions between the furan ring and Trp246 likely constrain the receptor in an inactive state and preclude structural rearrangements required for activation [31]; thus, this compound is undesirable for studies of the active state of the receptor. When compared to the $\beta_{2} \mathrm{AR}$ and rhodopsin structures, the ligand binding pocket is located further 


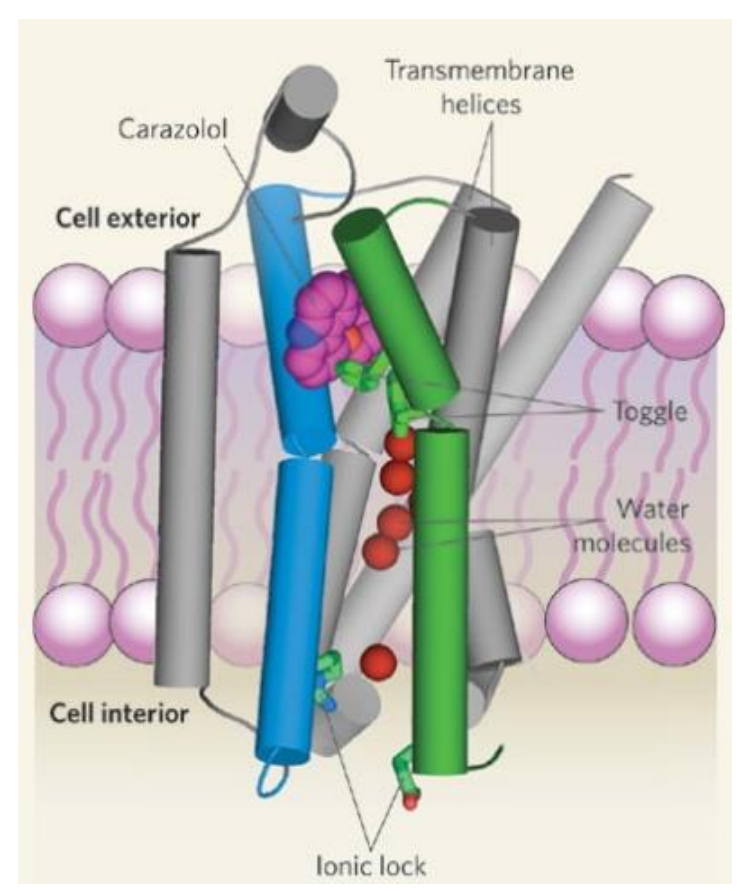

Figure 1.5. Receptor Toggle and lonic Lock. Schematic portrays critical elements of the $\beta_{2} A R$ structure, common to Class A GPCRs: a tryptophan 'toggle' and three-residue ionic lock on the intracellular face of the receptor. Reprinted by permission from Macmillan Publishers Ltd, Nature, Sprang, S.R., Structural biology: a receptor unlocked, copyright 2007.

away from the modeled position of the plasma membrane, with a nearly equal portion of the ligand exposed to extracellular solvent as buried [31] (Figure 1.2). The ligand in the $\mathrm{A}_{2 \mathrm{~A}} \mathrm{AR}$ structure is also turned such that its long axis lays perpendicular to the membrane, unlike the ligands in the other GPCR structures. In addition, the antagonist binding cavity is located closer to $\mathrm{H} 6$ and $\mathrm{H} 7$, as opposed to $\mathrm{H} 3$ and $\mathrm{H} 5$ as in the rhodopsin and adrenergic receptor structures [31]. The preceding findings regarding differences in the ligand binding pockets are significant for drug development, as the binding cavities cannot be generalized between receptor subclasses [63]. Due to these differences, in silico methods are unlikely to find effective solutions, and a focus should be placed on bringing unsolved structures to 
light. Comparison between multiple agonist-bound GPCR structures has been revealing. Despite significant differences in the ligand binding pockets of the $\beta_{2} \mathrm{AR}$, opsin and the $\mathrm{A}_{2 \mathrm{~A}} \mathrm{AR}$, agonist-binding induces similar changes in the overall core morphology, namely, tilting of $\mathrm{H} 7$, an extracellular shift of $\mathrm{H} 3$, a toggle of the intracellular regions of $\mathrm{H} 5$ toward the core as well as a $1.8 \AA$ tilt of TM6 toward the receptor core [64].

\section{The E/DRY Ionic Lock}

The ionic lock is an electrostatic interaction between a largely conserved triad of charged residues located near the intracellular side of the receptor, which tethers together $\mathrm{H} 3$ and $\mathrm{H} 6$ in the inactive state of the receptor [65], seen in (Figure 1.5). Mutations in the triad tend to increase the constitutive activity of $\beta_{2} \mathrm{AR}$ [9], suggesting that in the active conformation that the triad is in the unlocked position. In the crystal structure of $\beta_{2} A R$ with an inverse agonist thought to promote the inactive state, the ionic lock was broken [66]. This unexpected finding may explain why, unlike the rhodopsin structure with an intact lock (and which has no constitutive activity in the inactive state), the $\beta_{2} \mathrm{AR}$ may be able to signal constitutively at low levels [28]. Like the $\beta$ AR structures that have been solved, the $\mathrm{A}_{2 \mathrm{~A}} \mathrm{AR}$ crystal structure also reveals a broken ionic lock [31]. ICL2 in the $\mathrm{A}_{2 \mathrm{~A}} \mathrm{AR}$, as in the adrenergic receptor structures, is restrained by other interactions. The presence of a short helix in $A_{2 A} A R$, as well as in the turkey $\beta_{1}$ adrenergic receptor, and not in the other structures, correlates with the basal activity of $A_{2 A} A R$ and $\beta_{1} A R$. This short helix maintains a hydrogen bond between a conserved tyrosine within the helix and an ASP on H3 [31]. As the arginine of the E/DRY motif does not participate in the ionic lock, it may instead be stabilizing the deprotonated state of the proximal glutamate, which would strengthen 
the polar interactions between the E/DRY motif and the small helix of ICL2 [31]. It is thought that these interactions may be directly involved in G-protein activation [67].

\section{Membrane Localization}

Lipid rafts are membrane microdomains enriched in cholesterol and sphingolipids. Caveolae are even more specialized microdomains containing high concentrations of the scaffolding protein caveolin. The $\beta_{2} \mathrm{AR}$ has been shown to associate with caveolin 3 in neonatal myocytes [68]. Cholesterol-dependent compartmentalization has been observed with the opioid receptors $\mu$ and $\delta[69]$ as well as the $\mathrm{D}_{1}$ dopamine receptor [70] and the oxytocin receptor [71]. There is debate, however, whether these regions act as GPCR signaling platforms [72]. In BRET experiments examining the distribution of $\beta_{2} \mathrm{AR}$, the receptor was not concentrated in the microdomains; furthermore, cholesterol increase and caveolin expression inhibited $\beta_{2} \mathrm{AR}$ activity [73]. A further question is whether functional dimerization, mediated in part by cholesterol, is location-dependent. As mentioned previously, ORPM1 cholesterol-depletion studies demonstrated decreases in both G $\alpha$ binding and receptor dimerization [12]. Further work examining GPCRs in the lipid membrane is necessary to understand the relationship between G-protein binding, dimerization, and localization. 


\section{Lipid Interactions}

\section{Palmitic Acid}

The crystal structure of the $\beta_{2} \mathrm{AR}$ dimer revealed the presence of palmitate and cholesterol moieties at the dimer interface [9]. This co-localization suggests a possible relationship between palmitoylation and GPCR dimerization. Studies of the opioid receptor OPRM1 in which cholesterol was depleted or palmitoylation was abrogated, demonstrated a decrease in both $\mathrm{G} \alpha$ binding and receptor dimerization [12]. Two palmitoyl groups (Figure 1.6, yellow) and cholesterol (Figure 1.6, blue) are co-localized and tightly associated in the $\beta_{2} \mathrm{AR}$ structure [11], with the palmitoyl groups being in the expected location assuming palmitoylation of H8. As evident in the structure, there are additional cholesterol molecules (blue), and it would not be unreasonable to speculate that the palmitoyl group of $\mathrm{G} \alpha$ may initially dock to the receptor at this location. The $\mathrm{A}_{2 \mathrm{~A}} \mathrm{AR}$ is not palmitoylated on the $\mathrm{C}$-terminus of $\mathrm{H} 8$, and $\mathrm{H} 8$ is stabilized by interactions with $\mathrm{H} 1$ instead of the membrane in the antagonist-bound crystal structure [31]. It is unknown which local perturbations in the structure are caused by membrane binding. Engineered palmitoylation of $\mathrm{A}_{2 \mathrm{~A}} \mathrm{AR}$ suggests that lipid modification of the receptor may be involved in targeting to lipid rafts [74]. Unlike the $\mathrm{A}_{2 \mathrm{~A}} \mathrm{AR}$, the $\mathrm{A}_{1} \mathrm{AR}$ is palmitoylated. Relatively little is known regarding whether palmitoylation of the receptor is associated with the binding specificity of the G-proteins; however, it is known that palmitoylation does not affect receptor-effector coupling [75]. 


\section{Cholesterol}

The $2008 \beta_{2} \mathrm{AR}$ crystal structure by Hanson et al. contained two bound cholesterol molecules in a groove formed by regions of $\mathrm{H} 1 \mathrm{H} 2, \mathrm{H} 3$ and $\mathrm{H} 4$ [11]. The locations of the two cholesterol molecules are identical to those seen in the $\beta_{2} \mathrm{AR}$ dimer by Cherezov et al, (Figure 1.6) [9]. This binding is consistent with a cholesterol consensus motif that is conserved in class A GPCRs [60]. A cholesterol binding motif has also been reported for

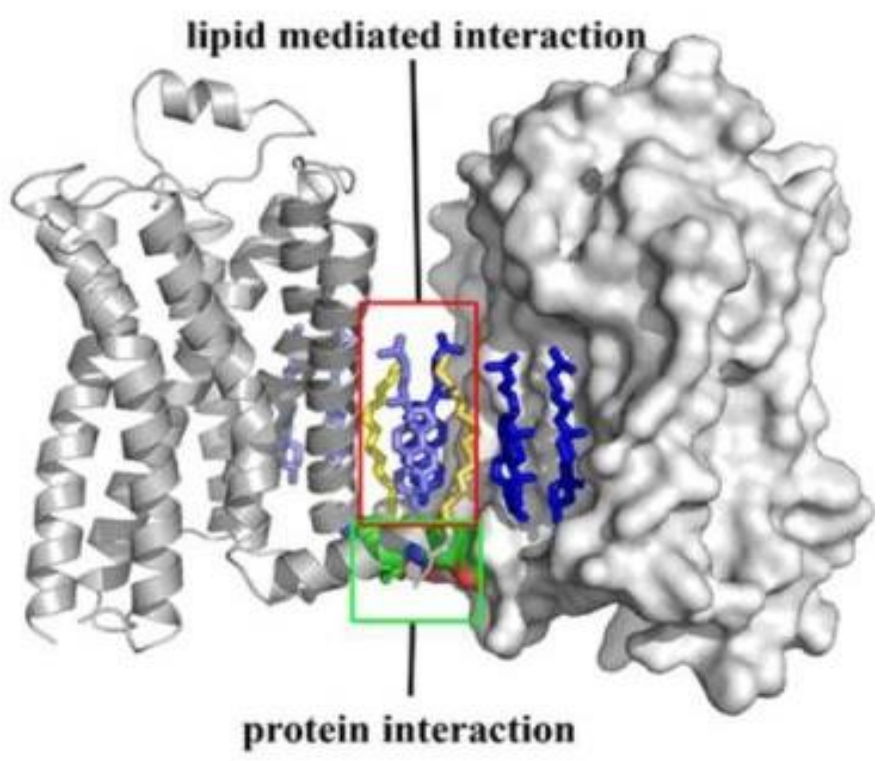

Figure 1.6. Lipid Mediated Dimer Interface of the $\beta_{2} A R$. Cholesterol (blue) and Palmitic Acid (yellow), protein interaction (green). From Cherezov, V., et al., Highresolution crystal structure of an engineered human beta2-adrenergic $G$ protein-coupled receptor. Science, 2007. 318(5854): p. 1258-65. Reprinted with permission from AAAS.

serotonin 1 receptors [76] and predicted for 44\% of Class A receptors [11]. Within the $\beta_{2} \mathrm{AR}$ dimer, a network of ionic charge interactions form the only inter-receptor protein contacts [9]. The interface is composed of two cholesterol molecules and two palmitic acid molecules; $73 \%$ is ordered lipid [11]. It is unsurprising therefore that cholesterol was required for the crystallization of the $\beta_{2} \mathrm{AR}$ [9]. 
Biochemical data support a role for cholesterol as well. Depletion of cholesterol from neonatal cardiac myocytes altered $\beta_{2} \mathrm{AR}$ signaling [68], suggesting that cholesterol is necessary for proper receptor function. GPCRs are thought to be localized to cholesterolenriched membrane microdomains known as lipid rafts, thus, modifying cholesterol content may lead to alteration of subcellular signaling compartments, and consequently receptor localization. GPCRs IP and TP, which respond to prostacyclin and thromboxane, differ in their localization, IP in lipid rafts and TP excluded [77]. Cholesterol depletion or enrichment both suppressed signaling, for IP and the IPTP dimer, which also localized to lipid rafts, but not TP [77]. The combined evidence points to a functional role for cholesterol; its localization suggests a role in receptor oligomerization and possibly, association of the G-protein via lipid modifications.

\section{G-Proteins: Background and Significance}

\section{G-Protein Localization and Diversity}

16 known genes encoding $23 \mathrm{G} \alpha$ isoforms, 5 genes encoding $7 \mathrm{G} \beta$ isoforms, and 12 genes encoding $12 \mathrm{G} \gamma$ isoforms have been identified [78]. The four main classes of $\mathrm{G} \alpha$ are: $\mathrm{G} \alpha_{\mathrm{i}}, \mathrm{G} \alpha_{\mathrm{s}}, \mathrm{G} \alpha_{\mathrm{q}}$ and $\mathrm{G} \alpha_{12}$, based on sequence homology [35]. There is evidence that the heterotrimer forms within the endomembrane prior to reaching the plasma membrane [79, 80]. Ga subunits cycle between an inactive GDP-bound form and an active GTP bound form, with the receptor catalyzing the exchange. The affinity of GaGTP is much weaker than the affinity of G $\alpha$ GDP for G $\beta \gamma$, thus they dissociate after exchange. After dissociation, G $\alpha$ GTP and G $\beta \gamma$ bind downstream effectors. Although it is commonly accepted that G $\alpha$ is 
associated primarily with the plasma membrane, $\mathrm{G} \alpha$ has also been identified at the Golgi

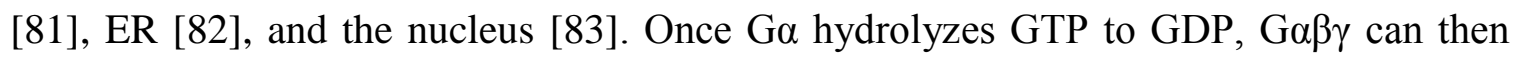
reform and the cycle begins again. G $\beta \gamma$ binds a large number of effectors that include $\mathrm{N}$, $\mathrm{P}$, and Q-type calcium channels [84], ion channels [85], $\beta$ adrenergic receptor kinase and other kinases [86], as well as phospholipase $A$ [87], phospholipase $C_{\beta 1-\beta 3}$ [88-90], adenylyl cyclase types I-VII [91-93], ADP ribosylation factors (ARFs) [94], and the membrane fusion synaptosomal-associated protein 25 (SNAP-25) [95].

\section{G-protein Signaling}

Downstream G-protein coupled signaling is mediated through the heterotrimeric G-

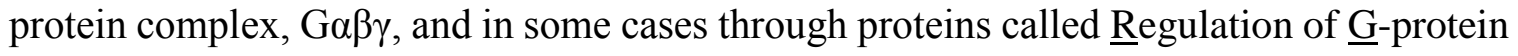
Signaling (RGS), a family of monomeric G-proteins that share structural homology with $\mathrm{G} \alpha$. It is the receptor interaction with the G-protein complex that modulates the downstream effects of receptor activation, and it is the receptor type interaction with a particular heterotrimer that determines the specificity of the response, although only a small number of in vivo combinations have been defined in their interaction with particular receptors. How specificity in the signaling response is achieved is an area of strong interest within the field. Figure 1.7 illustrates this signaling diversity [96]: G $\alpha_{\mathrm{i}}$ inhibits the production of cAMP from ATP via interaction with adenylate cyclase. G $\alpha_{\text {s }}$ is primarily involved in activation of the cAMP pathway by stimulating the production of cAMP from ATP during adenylate cyclase binding. $G \alpha_{q}$ binds phospholipase $C \beta$, which cleaves $\mathrm{PIP}_{2}$ into the second messengers, DAG and IP3. Ga $\alpha_{12}$ is involved in Rho signaling. Heterodimerization of the receptor, which will be discussed in more detail in a later section, 
is known to alter receptor localization and signaling. Heterodimers of the $\mathrm{A}_{2 \mathrm{~A}} \mathrm{AR}$ and the $\mu$-opioid receptor, when both activated, decrease phosphorylation of ERK1/2, while individually when activated, increase ERK1/2 signaling [97]. The molecular basis for how heterodimerization alters signaling in the G-protein is not entirely known; however, in the case of $\mathrm{A}_{2 \mathrm{~A}} \mathrm{AR}$ and $\mu$-opioid receptor heterodimers, binding of morphine to the $\mu$-opioid receptor provokes a conformational change in norepinephrine-bound $\mathrm{A}_{2 \mathrm{~A}} \mathrm{AR}$ that inhibits $\mathrm{G}_{\mathrm{i}}$ signaling [97].

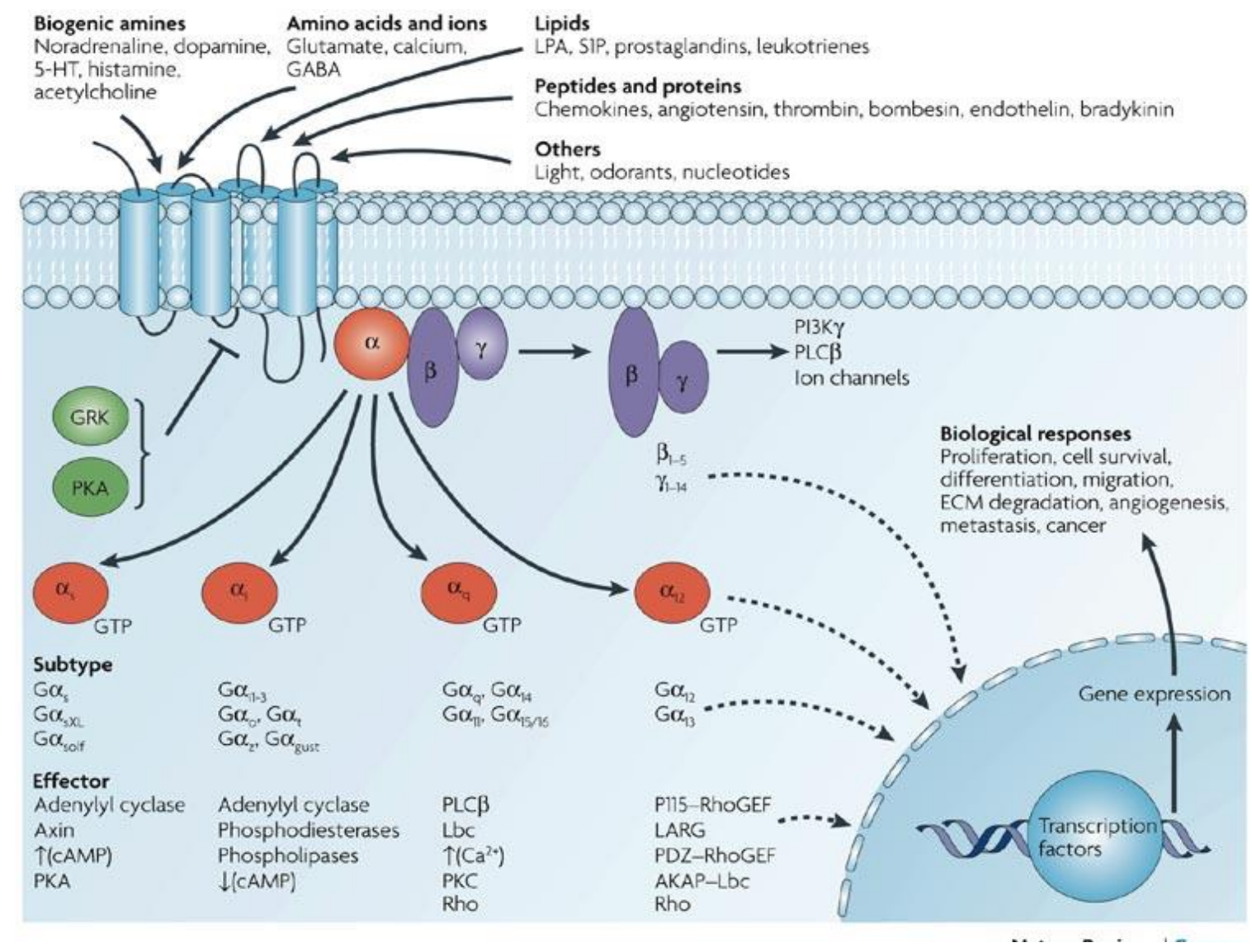

Figure 1.7. G-Protein Signaling Pathways. Reprinted by permission from Macmillan Publishers Ltd:, Nature Reviews Cancer, Dorsam, R.T. and J.S. Gutkind, G-protein-coupled receptors and cancer, copyright 2007. 


\section{G-protein Structure}

\section{$\mathbf{G \alpha}$}

$\mathrm{G} \alpha$ subtypes range in size from $39-52 \mathrm{kDa}$ and vary widely in their sequence identities (from 35-95\%) [98]. Ga is comprised of two domains, a GTPase domain and an $\alpha$-helical domain (Figure 1.8). Structurally, the GTPase domain is comprised of a sixstranded $\beta$-sheet surrounded by five $\alpha$-helices. Highly conserved sequences include the guanine nucleotide binding consensus sequences encoding five loop regions, a $\mathrm{Mg}^{2+}$ binding domain, and guanine-ring binding motif [99]. The three "switch regions" are also contained within this domain; these regions will be discussed in detail in a later section. Very little has been concluded about the amino $(\mathrm{Nt})$ and carboxyl termini $(\mathrm{Ct})$ because they are either removed or disordered in the crystal structures of the $\mathrm{G} \alpha$ monomers; however, according to mutation studies, they are responsible for receptor binding specificity and play a crucial role in G-protein activation [100]. In the available $G_{i}$ heterotrimer crystal structures, the Nt is $\alpha$-helical and ordered via interaction with G $\beta$ [15], as seen in Figure 1.8. However, a $30 \AA$ EM structure by Zhang et al. of the lipid modified native $G \alpha_{t}$ heterotrimer [101] does not show density consistent with an extended Nt-helix. Instead, the

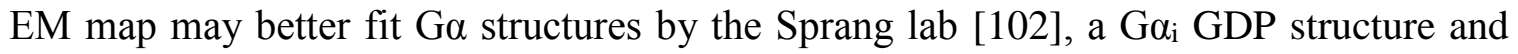
another in which they captured a structural intermediate which has GDP bound with a $\mathrm{Mg}^{2+}$ ion and a sulfate ion, which is taking the place of the terminal phosphate in the GTP-bound structure [103]. These structures show that the Nt forms a microdomain that sequesters the $\mathrm{Ct}$ when not bound to $\mathrm{G} \beta \gamma$ (Figure 1.8). If this structure is physiologically relevant, it would necessarily imply that the $\mathrm{Nt}$ is involved in regulating the binding of the $\mathrm{Ct}$ to the 
receptor. The involvement of the $\mathrm{Nt}$ in such regulation would be intuitive given that the $\mathrm{Nt}$ is responsive to the nucleotide-bound state of Ga. Even more intriguing is the structure of $\mathrm{AlF}_{4}$-activated $\mathrm{G} \alpha_{\mathrm{i}}$ bound to RGS4; this structure is thought to mimic the active GTPbound form of $\mathrm{G} \alpha$ [104] and shows an extended helix for the $\mathrm{Nt}$ similar to that seen in the heterotrimer crystal structures, but places it in an orientation that would insert into the membrane. An analysis of the hydropathicity index for the amino acid sequence corresponding to the GaNt suggests that it is favorable for membrane insertion [J.N. Wingard, unpublished data]. The most likely explanation is that the C-terminal loop preceding the helix is flexible, but these findings call into question that the heterotrimer crystal structures are relevant to the receptor-absent state and supports the possibility that the extended helix may instead be relevant to the receptor-associated Ga structure.

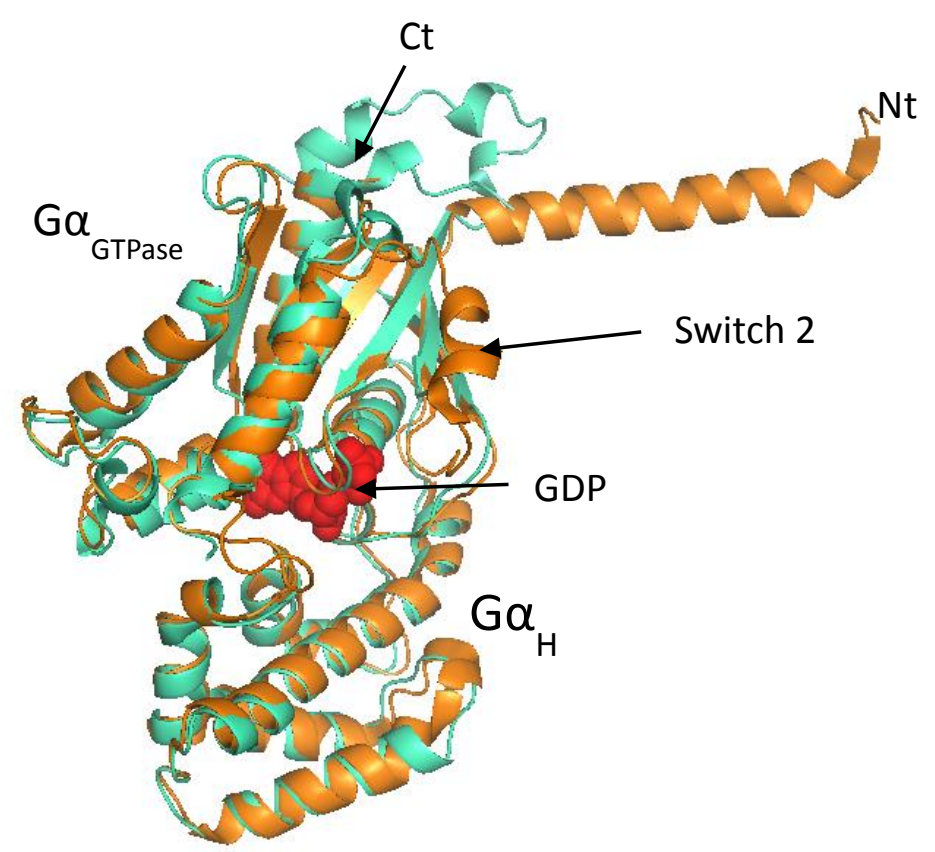

Figure 1.8. Structural Comparison of the G $\alpha$ monomer and $G \beta \gamma$-bound $G \alpha$. GDP-bound $G \alpha$ monomer shown in green (1GDD) and GDP-bound Ga shown in orange (1GP2). GDP is depicted in red. The $\mathrm{G \alpha}$ GTPase and $\mathrm{G} \alpha_{H}$ of $\mathrm{G} \alpha$ are noted. 
All $G \alpha$ subtypes, with the exception of $G \alpha_{\mathrm{t}}$ are post-translationally palmitoylated via a reversible thioester bond at cysteines proximal to the Nt. The $\mathrm{G}_{\mathrm{i}}$ family contains an additional irreversible myristoylation via co-translational addition of myristate to an $\mathrm{N}$ terminal glycine following removal of the terminal methionine [105]. Myristoylation of the $\mathrm{Nt}$ has also been shown to modulate interactions between $\mathrm{G} \alpha$ and effectors $[106,107]$. $\mathrm{G} \alpha_{\mathrm{q}}$ and $\mathrm{G} \alpha_{12}$ are only palmitoylated, not myristoylated [108].

\section{G及}

$\mathrm{G} \beta$ is $36 \mathrm{kDa}$ and contains no lipid modifications; sequence identity is less than in $\mathrm{G} \alpha(50-90 \%)$, and there is added diversity due to splice variants. It is a seven-bladed propeller structure in which each blade is comprised of four antiparallel $\beta$-strands, wherein a WD40 sequence motif contributes to the last three strands of one blade and the first of the next. The $\mathrm{Ct}$ of $\mathrm{G} \beta$ contributes to the fourth $\beta$-strand on the last blade, closing the ring. $\mathrm{N}$-terminal to the last strand of the propeller is a coiled-coil formed by the interaction of G $\beta$ with $\mathrm{G} \gamma[109,110]$, important for correct folding and function [111, 112] (Figure 1.9).

\section{G $\gamma$}

$\mathrm{G} \gamma$ is small, between only 7-8 kD, and subtypes share even less sequence identity (30-80\%) than the G $\beta$ subtypes [98]; it is composed of a simple pair of $\alpha$-helices connected by a loop. The Nt of $\mathrm{G} \gamma$ interacts with the Nt of $\mathrm{G} \beta$, while the $\mathrm{Ct}$ binds to Blade 5 and Blade 6 of the $\beta$-propeller structure [110], as seen in (Figure 1.9). Like the Ga subunit, the $\mathrm{G} \gamma$ subunit is also lipid-modified. All G $\gamma$ are post-translationally isoprenylated at their Ctermini through a stable thioether bond to the cysteine in the $\mathrm{CaaX}$ motif. The three $\mathrm{C}$ - 
terminal residues are removed and the C-terminal cysteine is subsequently carboxylmethylated, increasing the G $\beta \gamma$ affinity for the membrane [113]. G $\gamma$ is either farnesylated (if $\mathrm{X}=$ serine, methionine, glutamine or alanine) or geranylgeranylated (if $\mathrm{X}=$ leucine). $\mathrm{G} \gamma$ is also methylated at the $\mathrm{C}$-terminal cysteine, and its effect on its interaction with the receptor is unknown [114]. Despite high sequence identity, not all combinations of the approximately $60 \mathrm{G} \beta \gamma$ combinations can form. Different G $\beta \gamma$ combinations are able to bind the same Ga subtype however [35], suggesting that binding specificity may be driven by expression or localization.

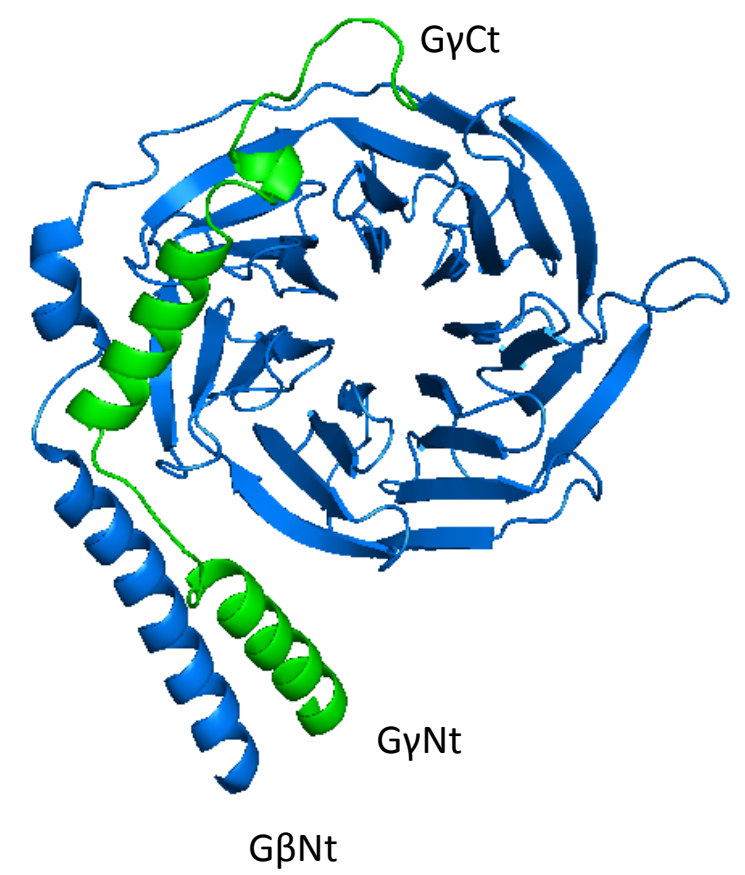

Figure 1.9. Propeller structure of $G \beta \gamma . G \beta$ in blue and $G \gamma$ in green form a coiled coil at their N-termini (1GP2). The lipid-modified C-terminus of $\mathrm{G}_{\gamma}$ is located proximal to the membrane. The narrow opening of the propeller seen in the center binds the small $\mathrm{Ga}$ helix comprising Switch 2 in the heterotrimer. 
Chimera studies have shown that the $\mathrm{G} \gamma \mathrm{C}$-terminus and prenyl group are important for coupling $\mathrm{G} \alpha$ to the receptor [115] as they form multiple contacts on the $\mathrm{G} \alpha$ subunit and a C-terminal region of the $\mathrm{G} \beta$ subunit. According to studies of $\mathrm{G} \alpha$ chimeras, as well as farnesyl and geranylgeranyl switching studies [114], isoprenyl modification of $\mathrm{G} \gamma$ is critical to receptor specificity with the C-terminal sequences being the most important. $\mathrm{G} \beta \gamma$ more effectively couples $G \alpha_{t}$ to rhodopsin when $G \gamma$ is geranylgeranylated rather than farnesylated [114]. Effect of prenyl type is greater with $\mathrm{G} \gamma 1$ than with $\mathrm{G} \gamma 2$ [114], suggesting that the amino acid sequence does play a role in receptor binding. This is supported by mutational analyses [116]. However, this may not be due to direct effects of the amino acid sequence but rather by the sequence affecting placement of the lipid tail. Truncation experiments have demonstrated that the lipid modifications on the $\mathrm{G} \gamma$ and $\mathrm{G} \alpha$ subunits interact and that their termini are likely in close proximity [117]. The lipid modifications have also been shown to enhance the affinity of $\mathrm{G} \alpha$ for $\mathrm{G} \beta \gamma$ and are thought to stabilize the receptor interface $[112,118,119]$.

\section{Ga Switch Regions}

Guanine nucleotide exchange induces three specific conformational changes that respond to G-protein binding between subunits, the receptor and effectors; these regions, termed 'switches' are involved in sensing nucleotide identity and regulating the interaction of the G-protein with other molecules. Switches I and II are located at the G $\alpha-G \beta$ interface in the heterotrimer $[15,120]$. Switch 1 (Figure 1.10, S1) is a loop that connects the terminal helix of $G \alpha_{H}$ to the $\beta 2$ strand of the GTPase domain. When GDP is exchanged for GTP, Switch 1 is pulled towards the nucleotide binding pocket such that it forms new bonds with 
$\mathrm{Mg}^{2+}$ and the $\gamma$-phosphate of GTP $[15,121]$. In addition, Switch I plays a critical role in binding RGS proteins [122]. Switch 2 (Figure 1.10, S2) is comprised mainly of a small helix that forms the interface between the G $\alpha$ and G $\beta$ subunits; it is disordered in the GDPbound form and becomes ordered upon GTP binding [15, 122, 123]. Switch 2 is also more flexible in the GaGTP state than the GDP-bound state [120], which may have important implications for effector binding. Switch 3 is a loop that caps the intracellular face of the nucleotide binding pocket. Upon G-protein binding to the receptor, all three switch regions collapse towards the $\gamma$-phosphate of GTP [124], thus destabilizing the interaction of G $\alpha$ with G $\beta \gamma$ and initiating dissociation.

\section{G-Protein Coupled Receptor and G-Protein Interactions}

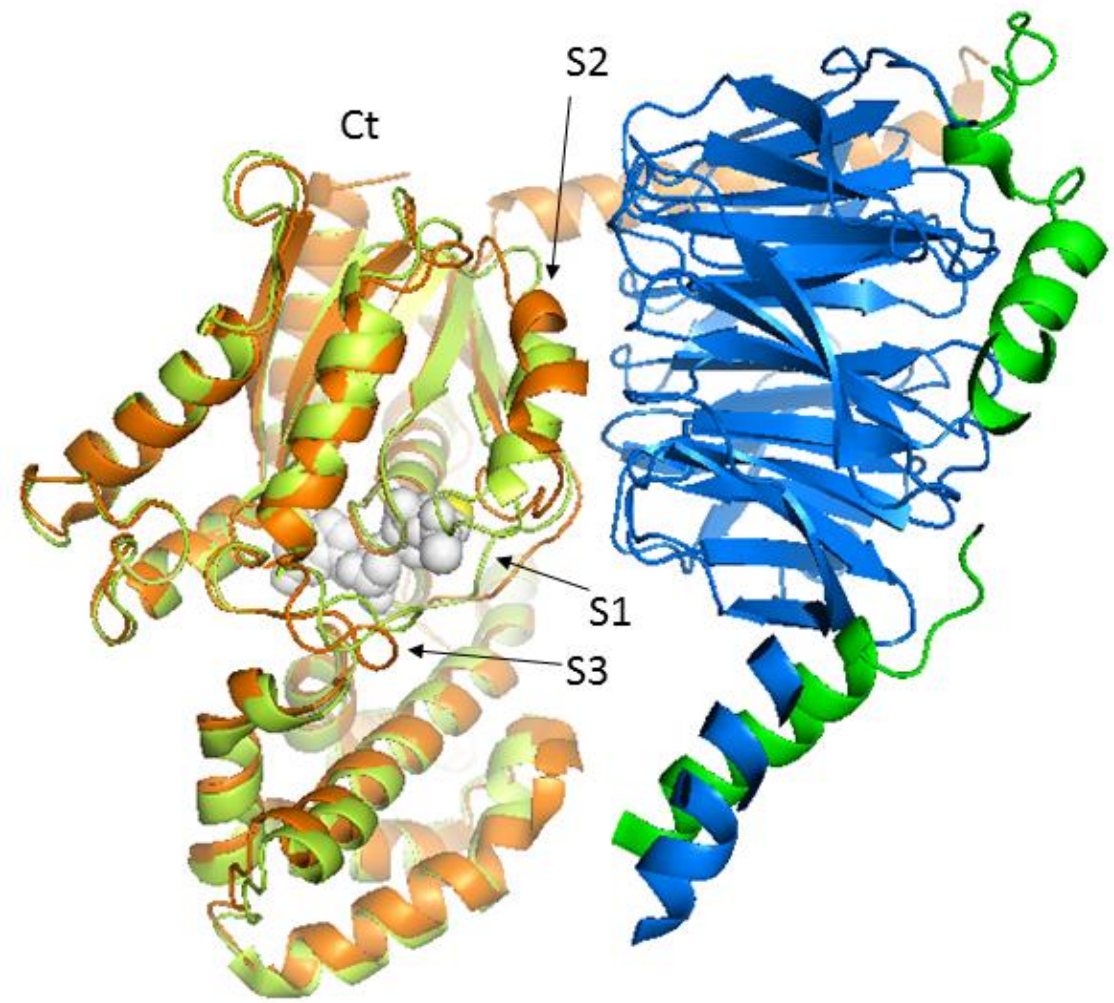

Figure 1.10. G $\alpha$ Switch Regions. GDP-bound $G \alpha_{i}$ heterotrimer (1GP2) with $G \alpha$ in chartreuse, $G \beta$ in blue, and $G \gamma$ in green aligned with GTPyS-bound $G \alpha(1 G I A)$ in orange. 


\section{G-protein Receptor Contacts}

The intracellular face of GPCRs contains seven $\alpha$-helices, three interhelical loops and a fourth loop to a short entirely intracellular eighth helix (H8) at the C-terminus, containing a palmitoylation site [125]. Highly conserved domains include $\mathrm{H} 8$, the palmitoylation site, and the EDRY and NPxxYx5F motifs. Activation in rhodopsin results in an outward movement of H6, which opens a cleft on the intracellular side of the receptor [126] that is essential for activation of the G-protein. This has been shown by crosslinking of disulfides or metal-ions between $\mathrm{H} 2$ and $\mathrm{H} 6$ that prevented light-induced nucleotide exchange in transducin $[126,127]$. The $\mathrm{Ct}$ helix of $\mathrm{G} \alpha$ fits into the intracellular cleft of the receptor with the axis of helix $\sim 40^{\circ}$ to the membrane normal based on NMR data [126, $128]$.

Both $\mathrm{Nt}$ and $\mathrm{Ct}$ residues of $\mathrm{G} \alpha$ interact with GPCRs. A peptide corresponding to the third intracellular loop of the $\mathrm{A}_{2 \mathrm{~A}} \mathrm{AR}$ crosslinks to the $60 \mathrm{~N}$-terminal amino acid residues of $\mathrm{G} \alpha[126,129]$. Kisselev demonstrated that a farnesylated 12 residue peptide, which forms an amphipathic helix on activation at the $\mathrm{G} \gamma \mathrm{Ct}$, can also stabilize the Meta II conformation of rhodopsin [130]. The seven most C-terminal residues of $\mathrm{G} \alpha_{t}$ found to be most important to receptor coupling [131] were identified in the crystal structure of opsin [8]. Mutations in this region have been associated with receptor uncoupling, one of which is known to cause Albright's hereditary osteodystrophy [132, 133]. The Ga N-terminal helix has been shown to interact with the receptor based on mutagenesis [134], crosslinking [129], peptide competition and chimera studies [135]. G $\alpha$ peptides corresponding 
to the terminal $\alpha 5(\mathrm{Ct})$ helix of $\mathrm{G} \alpha$ bind receptors with high affinity and are able to mimic G-protein stabilization of the active state of the receptor [136]. A peptide corresponding to the ten $\mathrm{C}$-terminal amino acid residues of $\mathrm{G} \alpha$ co-crystallized with opsin [8], suggesting an orientation for $\mathrm{G} \alpha$ with respect to opsin. Chemical crosslinking studies further showed binding of rhodopsin and D2 dopamine receptor to the $\mathrm{Ct}$ of $\mathrm{G} \alpha[137,138]$. The crystal structure of $\beta_{2}$ AR-Gs confirmed many of the biochemical findings, the primary receptor$\mathrm{G} \alpha$ contact at the $\mathrm{Ct}$, and contact with the $\mathrm{G} \alpha \mathrm{Nt}$ helix, $\alpha 4 / \beta 6$ and $\beta 2 / \beta 3$ loops [139]. Surprisingly, there were no receptor-G $\beta$ contacts.

\section{G-protein Activation}

\section{Docking}

Whether $\mathrm{G} \alpha$ initially binds the receptor prior to association with $\mathrm{G} \beta \gamma$ or in complex with G $\beta \gamma$ is a matter of some debate. Sulfhydryl modification of the C-terminal cysteine of $\mathrm{G} \alpha_{\mathrm{t}}$ blocks binding to photoactivated rhodopsin but not to dark rhodopsin [140], suggesting that $\mathrm{G} \alpha$ can only bind the receptor when the receptor is in the activated state, and in the absence of $\mathrm{G} \beta \gamma$. A high affinity interaction $(\sim 64 \mathrm{nM})$ between dark rhodopsin and $\mathrm{G} \alpha_{\mathrm{t}}$ [141] suggests that the binding site for $G \alpha_{t}$ in the receptor is saturated. Additionally, the $\mathrm{A}_{2 \mathrm{~A}} \mathrm{AR}$ co-purifies with $\mathrm{G} \alpha_{\mathrm{i}}$ [142], despite a 50-fold lower affinity in the absence of the heterotrimer. Addition of GTP $\gamma \mathrm{s}$ does not cause dissociation, suggesting that the receptor complexes with the GDP-bound heterotrimer [141]. Additional results have also demonstrated that $\mathrm{G} \alpha$-receptor complexes form independently of $\mathrm{G} \alpha \beta \gamma$ association [143]. 
The structure of opsin co-crystallized with a peptide corresponding to the $\mathrm{G} \alpha \mathrm{C}$ terminus [8] provided an initial basis for the G-protein activation model from Scheerer and Hildebrand [144]. Computational modeling predicted two distinct orientations for the Cterminal peptide with respect to the receptor. Taken together, the results suggest that binding of the C-terminus requires a step-wise 'tethering' association with the receptor wherein the $\mathrm{G} \alpha \mathrm{Ct}$ explores the intracellular receptor binding cavity (not the binding pocket for ligand). From the perspective of the receptor, fluorescence spectroscopy experiments demonstrate that the receptor undergoes at least two modes of conformational change, rapid and slow [145], with multiple rapid changes being associated with nucleotide exchange in the G-protein. NMR results corroborate a multi-step model in which GaGDP binding of $\mathrm{G} \beta \gamma$ induces two conformational states for the receptor-interacting $\mathrm{G} \alpha \mathrm{Ct}$, which may promote interactions with the receptor and pre-organize the guanine nucleotide binding pocket [146].

\section{Nucleotide Exchange}

The $\beta_{2}$ AR-Gs complex structure lacking bound nucleotide revealed a direct mechanism for nucleotide release during the exchange of GDP for GTP [6]. The receptor makes contact largely with the $\mathrm{Ct}$ of $\mathrm{G} \alpha$. Preceding the $\mathrm{Ct}$, is the $\alpha 5 / \beta 6$ loop. The end of the $\alpha 5 / \beta 6$ loop occludes the nucleotide binding site, thus, motion of the GaCt helix and movement of the $\alpha 5 / \beta 6$ loop is likely involved with GDP release [147]. EPR studies of G $\alpha$ also consistent with an explanation where the receptor promotes a rotation and translation of the $\mathrm{Ct}$ helix towards the base of the guanine nucleotide [148]. Insertion of a flexible linker at the beginning of $\alpha 5$ decouples receptor binding from nucleotide release, 
suggesting that movement of $\alpha 5 / \beta 6$ (which contains a TCAT sequence directly contacting the guanine ring) is involved in nucleotide release [148].

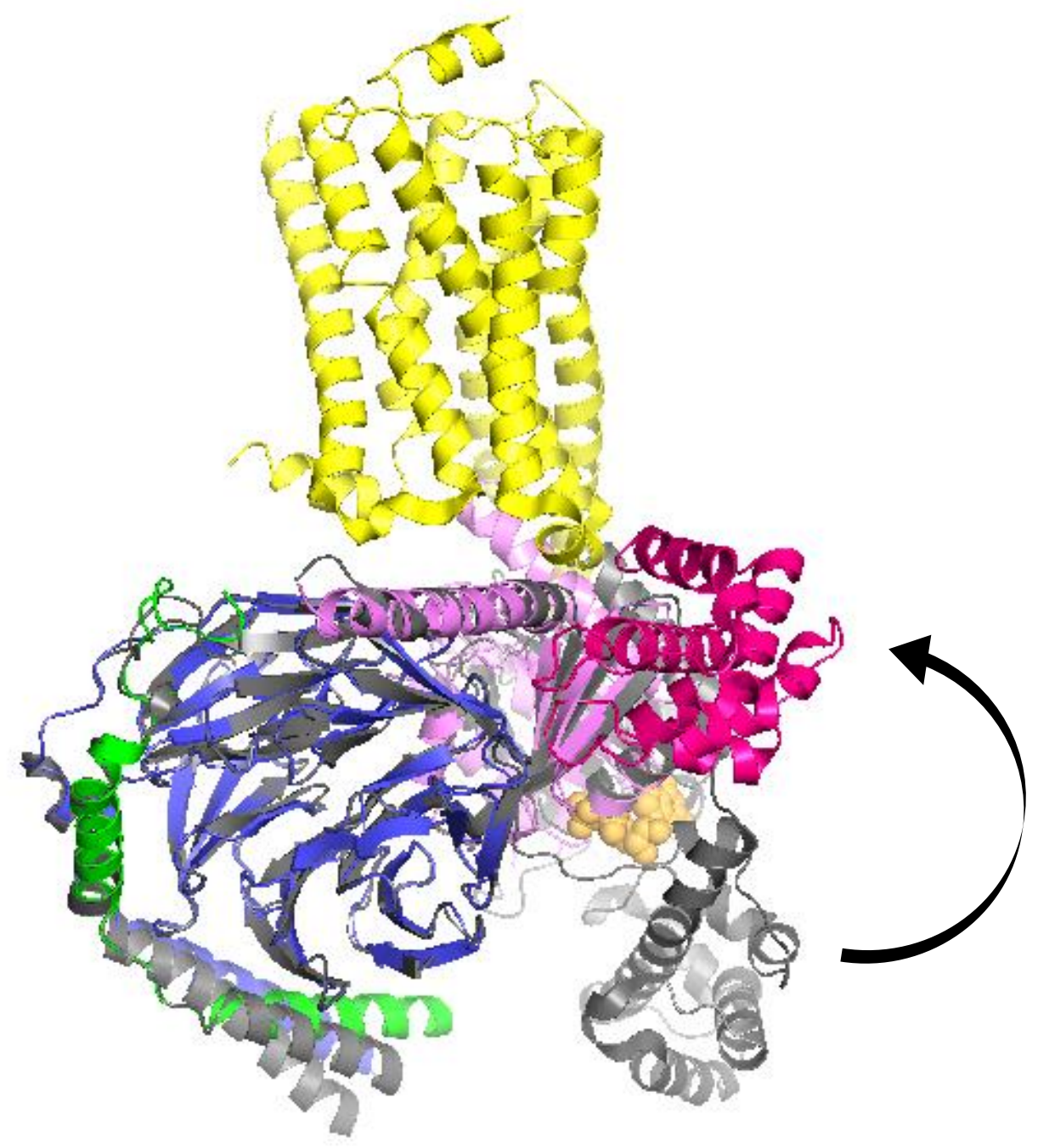

Figure 1.11. Nucleotide Exchange Related Conformational Changes in the G-Protein During Nucleotide Exchange. GDP-bound heterotrimer (1GP2) depicted in gray, and GDP in orange. $\beta_{2} A R-G s$ (3SN6) colored to indicate subunits: receptor (yellow), G $\beta$ (blue), GY (green), $G \alpha_{H}$ (fuchsia), $G \alpha_{N t}$ and $G \alpha_{G T P a s e}$ (violet). Movement of the helical domain allows for free dissociation of GDP and association of GTP. 
According to fluorescence studies of reconstituted complexes between rhodopsin and $\mathrm{G} \alpha_{\mathrm{i}}$ in rod outer disc membranes, the activation signal is communicated via hydrophobic interactions from ICL2 to the C-terminal portion of the $\alpha 5$ helix and the $\beta 2 / \beta 3$

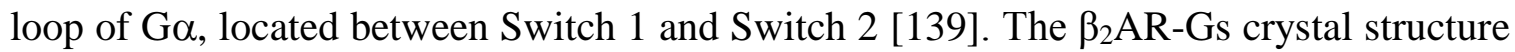
in the nucleotide-free state reveals that the $\alpha 5$ helix is rotated and displaced towards the receptor by $6 \AA$ and the $\alpha 5 / \beta 6$ loop that interacts with the guanine ring of the nucleotide is displaced away from the binding pocket [6]. The P-loop, which coordinates the phosphate in the nucleotide-bound form of $\mathrm{G} \alpha$ is also altered [6], as expected. Switch 1 is partially disordered in the $\beta_{2} \mathrm{AR}-\mathrm{Gs}$ nucleotide-free state. The conformational state of Switch 2 during nucleotide exchange is unknown, as a stabilizing nanobody is bound to a portion of it in the structure of the complex [6]. Most striking is the displacement of the Ga helical domain towards the membrane, opening up the nucleotide binding pocket in the $\beta_{2} \mathrm{AR}-\mathrm{Gs}$ crystal structure [6]. Critical to this motion is the action of the receptor on $\mathrm{G} \alpha$, where movement of transmembrane helices $\mathrm{H} 5$ and $\mathrm{H} 6$ propagate the signal through the $\mathrm{G} \alpha \mathrm{Ct}$ disrupting hydrogen bonds and converting a $\beta$-sheet to a flexible loop, removing constraints on the helical domain and allowing it to move in a hinge-like motion and providing for direct release of GDP (Figure 1.11).

\section{G-Protein Dissociation}

During GTP binding, there are significant changes in Switch 1 and Switch 2 [121]. Upon binding of GTP $\gamma \mathrm{S}$ to $\mathrm{G} \alpha$, fluorescence experiments suggest that Switch 1 maintains its receptor-activated conformational state; however, Switch 2 is reorganized and moves towards the GaGTPase domain [139]. These results suggest a similar environment for GTP 


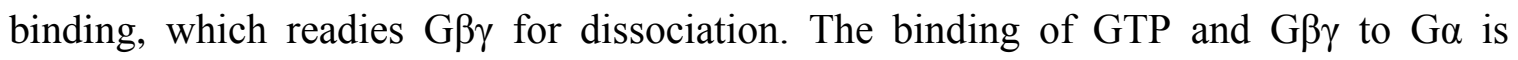
negatively cooperative [149], such that the receptor plays a role in creating a transient state that is favorable for the separation of $G \beta \gamma$ and $G \alpha$, stabilizing $G \alpha$ in a metastable conformation prior to GTP binding. Changes in Switch 2 following GTP binding likely facilitate dissociation. Rearrangement of the GaNt helix, as discussed previously, also appears to mediate dissociation.

\section{Receptor Oligomerization and G-protein Complex Stoichiometry}

A 1:1 stoichiometry of receptor to heterotrimer is sufficient for signaling [150]; however, a growing body of evidence suggests that GPCRs act primarily as dimers, and occasionally as higher order oligomers [151]. Dimerization, both homodimerization and heterodimerization of GPCRs, is now commonly accepted. In the case of certain GPCRs, such as the smoothened [152] and GABA [153] receptors, dimerization is necessary for Gprotein activation. As an example, the $\alpha 2 \mathrm{~A}$-adrenergic receptor ( $\alpha 2 \mathrm{~A}-\mathrm{AR})$ and the $\mu$-opioid receptor form heterodimers at the plasma membrane of neurons involved in pain sensation; studies in live cells have revealed that activation of either receptor leads to an increase in signaling [154]. This may explain why clonidine, an $\alpha 2 \mathrm{~A}-\mathrm{AR}$ agonist, has analgesic effects in its own right, in addition to potentiating the analgesic activity of opioids such as morphine [154]. Activation of either the $\alpha 2 \mathrm{~A}-\mathrm{AR}$ or the $\mu$-opioid receptor induces a conformational change within $400 \mathrm{msec}$ in its heterodimeric partner [97]. Elucidation of the functional unit of activity and a thorough understanding of the extent of heterodimerization is relevant to drug discovery efforts. 


\section{Receptor Oligomerization in Pathology}

Evidence exists that alteration of normal heterodimerization among GPCRs can be pathological, as with the bradykinin and angiotensin receptors. Receptor heterodimerization is known to contribute to preeclampsia [155]. $\beta_{2}$-bradykinin receptor levels are significantly increased in preeclampsia leading to increased heterodimerization between the $\mathrm{AT}_{1}$ angiotensin receptor and the $\beta_{2}$-bradykinin receptor, conferring increased sensitivity to angiotensin II [155]. A $\mathrm{A}_{2 \mathrm{~A}}$ adenosine receptors heterodimerize with the dopamine $\mathrm{D}_{2}$ receptor in the brain, thus making the adenosine receptor a target for therapeutics against Parkinson's disease [156]. Interestingly, Ferre and others demonstrated that $\mathrm{A}_{2 \mathrm{~A}} \mathrm{AR}$ and dopamine receptor heterodimers can act synergistically or antagonistically depending on the identity of the G-protein coupling and dopamine receptor

subtype [1]. It has been suggested that hypercholesterolemia may play a role in altered GPCR signaling, specifically in the prostacyclin and thromboxane receptors, leading to hypertension [77]. Monomerization of $\mathrm{GABA}_{\mathrm{B}}$ receptor dimers by the endogenous $\mathrm{GABA}_{\mathrm{B} 1-b i n d i n g}$ protein in neuropathic rats impairs signaling in spiny neurons; a peptide that restores dimerization, restores function and confers an increased sensitivity to antinociceptive therapy [157].

\section{Receptor Oligomerization Interfaces}

While dimerization of GPCRs is now commonly accepted, the dimerization interface(s) are a matter of much debate. Figure 1.12 summarizes much of the biochemical literature on the topic. Two prominent interfaces emerge, H8-H1 and H4-H5. Insight into GPCR dimerization has been derived by atomic force microscopy (AFM) studies of 
paracrystalline rhodopsin packing in native murine rod outer segment disc membranes. AFM images displayed ribbons of dimers with two dimerization interfaces, one with increased surface area and a secondary interface [4]. Subsequent to the availability of the high-resolution X-ray structure of rhodopsin and molecular dynamics simulations, packing models proposed two interfaces, H4-H5 and H8-H1 [158]. Despite predictions that H4-H5 would be the primary interface due to an increased buried surface area, molecular dynamics simulations suggest that the H8-H1 interface is more stable [158]. Rhodopsin may be considered an unusual GPCR because of its high concentration and paracrystalline packing in disc membranes, although other GPCRs have been shown to be active as higher order oligomers, such as the $\kappa$-opioid receptor, as revealed by BRET in live cells [13].

A recent model based on cysteine-crosslinking of mGluR2 suggests an alternative interpretation of the observation of multiple dimerization interfaces; it suggests that dimerization is a dynamic process involving rotation of the receptors during activation, converting the interface from H4-H5 to H5-H6 [159]. Synthesizing the structural data regarding GPCR oligomerization requires caution as oligomerization, as with ligand binding, may not be generalizable between classes. Chapter 5 will describe a speculative model for homo- and hetero-dimerization for Class A GPCRs based on EM studies of adenosine receptors by the author, as well as recently published 3D structures deposited in the PDB. 


\begin{tabular}{|c|c|c|c|}
\hline GPCR & Interface & Methods & References \\
\hline$\alpha 1 \mathrm{bAR}$ & $\begin{array}{l}\mathrm{H} 8-\mathrm{H} 1 \\
\mathrm{H} 4-\mathrm{H} 5\end{array}$ & FRET, Truncation & $\begin{array}{c}\text { Carrillo et al. } 2004 \\
\text { Lopez-Giminez et al. } 2007\end{array}$ \\
\hline$A 2 a-A R$ & $\mathrm{H} 4-\mathrm{H} 5$ & XRD & Liu et al. 2012 (4E1Y) \\
\hline k-opioid & $\begin{array}{c}\mathrm{H} 8-\mathrm{H} 1 \\
\mathrm{H} 4-\mathrm{H} 5 \\
\mathrm{H} 6\end{array}$ & XRD & Wu et al. $2012(4 \mathrm{DJH})$ \\
\hline$\mu$-opioid & $\begin{array}{l}\mathrm{H} 8-\mathrm{H} 1 \\
\mathrm{H} 5-\mathrm{H} 6\end{array}$ & XRD & Mangliket al. 2012 (4DKL) \\
\hline $5-\mathrm{HT} 2 \mathrm{C}$ & $\begin{array}{l}\mathrm{H} 8-\mathrm{H} 1 \\
\mathrm{H} 4-\mathrm{H} 5\end{array}$ & Disulfide trapping & Mancia et al. 2008 \\
\hline $5-\mathrm{HT} 4$ & $\mathrm{H} 4-\mathrm{H} 5$ & BRET & Berthouze et al. 2007 \\
\hline $\mathrm{C5a}$ & $\begin{array}{l}\mathrm{H} 8-\mathrm{H} 1 \\
\mathrm{H} 4-\mathrm{H} 5\end{array}$ & Disulfide trapping & Kico et al. 2003 \\
\hline CCR5 & $\begin{array}{l}\mathrm{H} 8-\mathrm{H} 1 \\
\mathrm{H} 4-\mathrm{H} 5\end{array}$ & FRET & Hernanz-Falcon et al. 2004 \\
\hline CXCR4 & $\mathrm{H} 4-\mathrm{H} 5$ & XRD & Wu et al. 2010 (3OEO) \\
\hline $\mathrm{D}_{2} \mathrm{DR}$ & $\begin{array}{l}\mathrm{H} 8-\mathrm{H} 1 \\
\mathrm{H} 4-\mathrm{H} 5\end{array}$ & $\begin{array}{l}\text { BRET, Crosslinking, FRET, } \\
\text { Truncation }\end{array}$ & $\begin{array}{l}\text { Lee et al. } 2003 \\
\text { Guo et al. } 2003 \\
\text { Guo et al. } 2005 \\
\text { Guo et al. } 2008\end{array}$ \\
\hline Metarhodopsin II & $\mathrm{H} 8-\mathrm{H} 1$ & 2D cryo-EM, XRD & $\begin{array}{l}\text { Ruprecht et al. } 2004 \\
\text { Choe et al. } 2011 \text { (3PXO) }\end{array}$ \\
\hline mGluR2 & $\mathrm{H} 4-\mathrm{H} 5$ & FRET, Immunoprecipitation & Gonzalez-Maeso et al. 2008 \\
\hline Opsin & $\mathrm{H} 8-\mathrm{H} 1$ & XRD & Parket al. 2008 (3CAP) \\
\hline Rhodopsin & $\mathrm{H} 8-\mathrm{H} 1$ & $\begin{array}{c}\text { 2D cryoEM, Crosslinking, } \\
\text { XRD }\end{array}$ & $\begin{array}{c}\text { Schertler et al. } 1995 \\
\text { Knepp et al. } 2012 \\
\text { Salom et al. } 2006(2137)\end{array}$ \\
\hline VT2R & $\mathrm{H} 4-\mathrm{H} 5$ & FRET & Mikhailova et al. 2008 \\
\hline
\end{tabular}

Figure 1.12. Observations of GPCR Oligomerization. XRD, X-ray diffraction. There are two predominant interfaces for GPCRs: $\mathrm{H} 8-\mathrm{H} 1$ and $\mathrm{H} 4-\mathrm{H} 5$. These interfaces would be located on opposite faces of the receptor. References are annotated with PDB ID for structures from XRD. 


\section{Conclusions and Aims}

Until 2011, and the publication of the $\beta_{2}$ AR-Gs crystal structure and accompanying single particle analyses, the mechanism of nucleotide release during G-protein activation by GPCRs was unknown. The $\beta_{2} \mathrm{AR}-\mathrm{Gs}$ structure also revealed the orientation of a nucleotide-free G-protein to a GPCR. It is reasonable to ask whether or not the G-protein activation mechanism is conserved amongst Class A GPCRs. Interpretation of single particle results for both the $\beta_{2} \mathrm{AR}-\mathrm{Gs}$ and rhodopsin-transducin complex was not straightforward. In addition, no contacts were observed between G $\beta \gamma$ and the receptor and it is possible that the presence of a stabilizing nanobody between $G \alpha$ and $G \beta \gamma$ in the $\beta_{2} A R-$ Gs complex may have altered their interaction. Further structural studies of additional receptor-G-protein complexes will be necessary to form robust conclusions regarding the stoichiometry of the receptor and its relationship to the G-protein.

An additional area of debate is the oligomeric state of the G-protein-bound receptor. Molecular dynamics simulations, cross-linking, 3D crystallization, BRET, FRET, and mutational analyses have suggested multiple interfaces for oligomerization among GPCRs. Identification of cholesterol binding sites and their presence at the dimerization interface in some GPCR structures point to their possible involvement in oligomerization. With respect to the adenosine receptors, there is very little known about the dimer interface. Computational studies of the $\mathrm{A}_{3} \mathrm{AR}$ using initial measurements derived from the packing of rhodopsin in native murine disc membranes identified $\mathrm{H} 4-\mathrm{H} 5$ as a primary dimerization interface and H8-H1 as a secondary interface [160]. However, there is no experimental evidence regarding the dimerization interface. Some inferences can be drawn from the 
packing of the adenosine receptor in the 3D crystal structures; however, most of the interfaces are non-physiological based on anti-parallel orientations of the receptors. Of the available $\mathrm{A}_{2 \mathrm{~A}} \mathrm{AR}$ structures, interfaces revealed by $3 \mathrm{D}$ crystallization include an antiparallel interface at H8-H1, a parallel interface at H4-H5 for $\mathrm{A}_{2 \mathrm{~A}}$-bRIL [161], a nonphysiological interface at H4-H5 due to the angle of contact [162], a non-physiological H5H6 interface due to a translation along the axis perpendicular to the membrane for an antibody-stabilized $\mathrm{A}_{2 \mathrm{~A}} \mathrm{AR}$ [163]. In addition, for two cases, crystal contacts were mediated by antiparallel H8-H1 and H5-H6 associations [64] [31]. The repeated incidence of the H8-H1, H4-H5, and H5-H6 interfaces is intriguing, as is the co-localization of cholesterol at these interfaces. Two cholesterol molecules bound to a hydrophobic groove on $\mathrm{H} 6$ form part of the crystal contact in the $\mathrm{A}_{2 \mathrm{~A} A R}$ [161]. Cholesterol has also been observed at the H8-H1 interface for the $\beta_{2} \mathrm{AR}[11]$ and is predicted to bind $\mathrm{H} 4$ of the $\mu$ opioid receptor [12]. Cholesterol also mediates formation of $\beta_{2} \mathrm{AR}$ crystals [9]. This is unsurprising at cholesterol comprises up to $25 \%$ of the cell membrane by weight. One may infer from the high frequency of antiparallel associations at the cholesterol-containing interfaces that while cholesterol may mediate dimer-formation, physiological specificity is conferred by other determinants. Examination of the adenosine receptor with cholesterol in the near-native milieu of a lipid bilayer may provide additional constraints necessary to identifying a physiologically-relevant dimerization interface.

To date, direct methods have not identified the adenosine receptor dimer interface. Does the adenosine receptor bind the G-protein as a monomer or dimer, and if as a dimer, 
what is the dimerization interface? This work has addressed these questions with two main objectives, the results of which will be addressed in Chapters 2 and 3 respectively.

1) To gain understanding about the molecular architecture of an adenosine receptorG-protein complex, specifically, the orientation of the receptor to the G-protein.

2) To obtain and utilize structural data to provide further insight into adenosine receptor function and oligomerization.

By the use of EM and the complementary techniques of 2D crystallography and single particle image analysis, the AR has been visualized in its dimeric as well as monomeric state. The results of this work discussed in Chapter 2 suggest that the detergent-solubilized $\mathrm{A}_{1} \mathrm{AR}$ and $\mathrm{A}_{2 \mathrm{~A}} \mathrm{AR}$ are monomeric. The $\mathrm{A}_{2 \mathrm{~A}} \mathrm{AR}$ is also monomeric when bound to the $\mathrm{G}$ protein, and single particle analysis suggests a tight interaction between the receptor and $\mathrm{G} \beta \gamma$, as well as flexibility of the $\mathrm{G} \alpha_{\mathrm{H}}$. Preliminary findings also suggest that G-protein activation provokes conformational changes in AR that increase its propensity to dimerize. 2D crystallization of the AR discussed in Chapter 3 reveals two dimerization interfaces, including one that is likely to be physiologically relevant. Image processing and analysis suggest that the primary dimerization interface for the $\mathrm{A}_{2 \mathrm{~A}} \mathrm{AR}$ is $\mathrm{H} 8-\mathrm{H} 1$. Interestingly, this interface is in agreement with molecular dynamics predictions. Chapter 4 will describe advances in a recently developed technique, micro electron diffraction (MicroED), which shows potential for solving structures of membrane proteins, such as the $\mathrm{A}_{2 \mathrm{~A}} \mathrm{AR}$, by direct electron diffraction. Finally, Chapter 5 will discuss structural implications in more detail and describe proposals for future developments in the field. In summary, this work provides 
insights into adenosine receptor dimerization, complex formation between the adenosine receptor and G-protein, and lays a foundation for more detailed molecular and functional studies of adenosine receptor dimerization and MicroED of 3D crystals of membrane proteins. 


\section{Chapter 2}

\section{Electron Microscopy and Single Particle Analysis of Adenosine Receptors and Receptor-G-protein Complexes}

\section{Introduction}

Challenges in the structural determination of large multi-protein complexes include expression levels of the protein components, purification and relevant stoichiometric assembly of stable complexes. Historically, large complexes have been excluded from NMR based on their size. 3D crystallization and XRD of large complexes is frequently complicated due to sample heterogeneity, conformational flexibility, and limited quantity of the sample, as well as crystal mosaicity arising from sample instability.

Electron microscopic techniques such as tomography and single particle analysis allow for structural determination of large macromolecular complexes without the need for crystals and circumvent many of the challenges and constraints of crystallization. While tomography and tomographic averaging is useful for resolving major structural features at low resolution (20-50 $\AA$ ), single particle analysis can theoretically permit a higher resolution analysis of smaller structural features. Combined with cryo-preservation, subnanometer resolution can be achieved [164].

The majority of structural knowledge regarding GPCRs has come from XRD studies of the receptor alone, thus we have a limited understanding of the molecular architecture of the GPCR-G-protein complex. 2D electron crystallography of a single 
GPCR, rhodopsin, have thus far been available to contextualize this information within the context of the lipid membrane. While we lack a structure of a membrane-associated GPCRG-protein complex, single particle analysis studies allow for examination of the complex without the constraints of crystallization.

Transmission electron microscopy involves a high voltage electron beam focused by magnetic 'lenses', with partial transmission of the beam at low electron dose recorded by CCD. Density variation in the sample results in scattering that contributes to the final projection images of the sample. Due to the low density differences between proteinaceous samples and buffer, for example, contrast or signal to noise is very low. To overcome this, negative staining is employed to enhance contrast. The stain is typically a heavy metal, uranyl acetate being one of the most common stains, yielding a dark background and a white appearing protein. The resolution limit is in part set by the grain size of the stain; for uranyl acetate, this is $13 \AA$. Although limited, sample deformation is possible, as well as other stain artifacts such as partial embedding.

Cryo-EM is a method that precludes such artifacts. By embedding the sample in vitreous ice, the resolution is no longer limited by the grain size of the stain, and stainrelated flattening of features can be avoided. While not necessary to obtain surface projections, as TEM is considered a direct imaging method, additional mathematical utilities or in silico methods can be used in concert to generate near atomic-resolution 3D reconstructions of cryo-preserved samples [165]. 
Single particle analysis takes advantage of a well-distributed set of orientations within the particle set. The particles are masked from individual micrographs by manual selection or by using automated procedures, their orientations estimated, the background subtracted, grouped and averaged, and then the data are back-projected to reconstruct the 3D particle, using a suite of image processing programs, EMAN2 [166]. Single particle analysis is subject to artifacts arising from preferred orientations of particles, and particle heterogeneity. With regards to processing, it is also possible to have issues arising from the initial model, as it is built $a b$ initio. It is possible to perform image processing starting with a known model; however, model-bias then becomes problematic. Samples with strong asymmetric features are advantageous in avoiding these issues. These features may be internal, or may be supplemented by way of Fab-labeling [167] or gold-labeling [168] with sufficiently large constructs. The method has some advantage over 3D crystallization and $\mathrm{XRD}$ in that samples are at a much lower concentration than necessary for crystallization and the resultant structures are not subject to packing artifacts sometimes found in 3D crystals. However, this low concentration may likewise not be physiological, and may result in the monomerization of higher order forms, especially under high detergent concentrations often required to solubilize membrane proteins.

\section{Insights from single particle analysis of other GPCRs}

Along with this work, which describes an $\mathrm{A}_{2 \mathrm{~A}} \mathrm{AR}-\mathrm{Gs}$ complex, there are two additional GPCR-G-protein complexes that have been examined using single particle analysis, rhodopsin-transducin and $\beta_{2}$ AR-Gs. In both cases, a nucleotide-free complex was generated. Activated rhodopsin can catalyze nucleotide exchange for multiple transducin 
molecules [169]. Rhodopsin can exist as dimers when reconstituted in lipid bilayers, as demonstrated by 2D crystallography [170], and examination of murine disc membranes of rod outer segments by AFM shows that rhodopsin is present in dimers as well as in higher oligomeric forms. [4].

Single particle examination of negatively stained $\beta_{2}$ AR-Gs guided the crystallization of the complex [6]. Westfield et al. examined a nanobody-stabilized nucleotide-free T4L- $\beta_{2}$ AR-Gs. The nanobody (a camelid-derived antibody of $\sim 15 \mathrm{kD}$ comprised of a single variable heavy-chain domain) Nb37 binds the Ga helical domain, $\mathrm{G} \alpha_{\mathrm{H}}$. The $\beta_{2} \mathrm{AR}-\mathrm{Gs}$ had strong preferential orientation, with the complex laying with its long axis on the grid surface. Examining the class averages of the 2D projections, with and without $\mathrm{Nb37}$, revealed multiple angular relationships of the $\mathrm{G \alpha H}$ to the receptor, suggesting flexibility between the $G \alpha_{H}$ and $G \alpha_{G T P a s e}$ domains [7]. Although some of this apparent flexibility may be due to a difference in the rotational angle relative to the particle orientations on the grid surface, taken together with the crystal structure of the complex, this flexibility allows for direct exchange of GDP for GTP during G-protein activation. While the crystal structure revealed a monomeric receptor, interpretation of the density attributed to the $\beta_{2} \mathrm{AR}$ was not so straightforward. The receptor density often appears large and bi-lobed, despite only one visible density for T4L per complex. The additional density has been attributed to a large detergent micelle [6].

Single particle analysis of rhodopsin-transducin also showed a bi-lobed density for the receptor, rhodopsin. Unlike the $\beta_{2} \mathrm{AR}$, which used an $\mathrm{N}$-terminal T4L fusion as a 
fiducial, rhodopsin was unlabeled. The predominant particles observed (40\% of total) were $130+/-6 \AA$ in length, the expected size of the complex [171]. In their initial single particle analysis, the authors used dark field STEM (scanning transmission electron microscopy) measurements to support their interpretation of a dimeric receptor bound to a single heterotrimer. Albeit at low resolution, the envelope readily accommodates two rhodopsin molecules; however, the density representing the receptor is asymmetrical in the plane of the membrane, which would not be expected for a dimer. Rhodopsin is glycosylated via aspartic acid and can bind concanavalin (conA) 1:1. In separate single particle analyses, the authors examined rhodopsin-conA complexes that also revealed a dimeric rhodopsin bound to a single G-protein [172] [173]. In contrast to the previous study, class averages showed a largely symmetrical dimer. The detergent LMNG was used instead of DDM in this second set of 3D reconstructions for rhodopsin-transducin. The size of the LMNG micelle was much smaller for rhodopsin-transducin than that for $\beta_{2} \mathrm{AR}-\mathrm{Gs}$, for which the authors could not offer a solution [172].

\section{Results}

\section{In silico experiments}

Much of this work was completed prior to the publication of the aforementioned single particle analysis studies of rhodopsin-transducin and $\beta_{2}$ AR-Gs complex; thus, it was necessary to generate a model to guide particle selection and other stages of image processing. To do this, I generated the model seen in Figure 2.1 using Pymol; the $\mathrm{A}_{2 \mathrm{~A}} \mathrm{AR}$ crystal structure was computationally aligned via Pymol to the crystal structure for opsin 
bound to the C-terminal peptide of $G \alpha_{t}$ [8]. The crystal structure of $G \alpha_{s} G T P$ was then visually aligned to the $\mathrm{Ct}$ peptide, taking into consideration the three missing $\mathrm{Ct}$ residues from the $\mathrm{G} \alpha_{\mathrm{s}} \mathrm{GTP}$ structure and the electrostatic potentials from vacuum electrostatics surface maps. The crystal structure of $\mathrm{G} \alpha_{\mathrm{i}} \beta 4 \gamma 2$ was then aligned to $\mathrm{G} \alpha_{\mathrm{s}}$ to provide a model for the interaction of the heterotrimer. Because density was not seen for the extended $\mathrm{Nt}$ helix of $G \alpha_{s}$ in the crystal structure, the $G \alpha_{s}$ Nt helix was modeled in based on the $G \alpha_{i}$ heterotrimer structure and oriented in a reasonable location with respect to the receptor. The final model was then energy minimized using the molecular graphics program Chimera. The boundaries of the lipid bilayer, defined by the white discs, were obtained from coordinates provided by the University of Michigan OPM (Orientations of Proteins in the Membrane) database (http://opm.phar.umich.edu/). The energy minimized structure

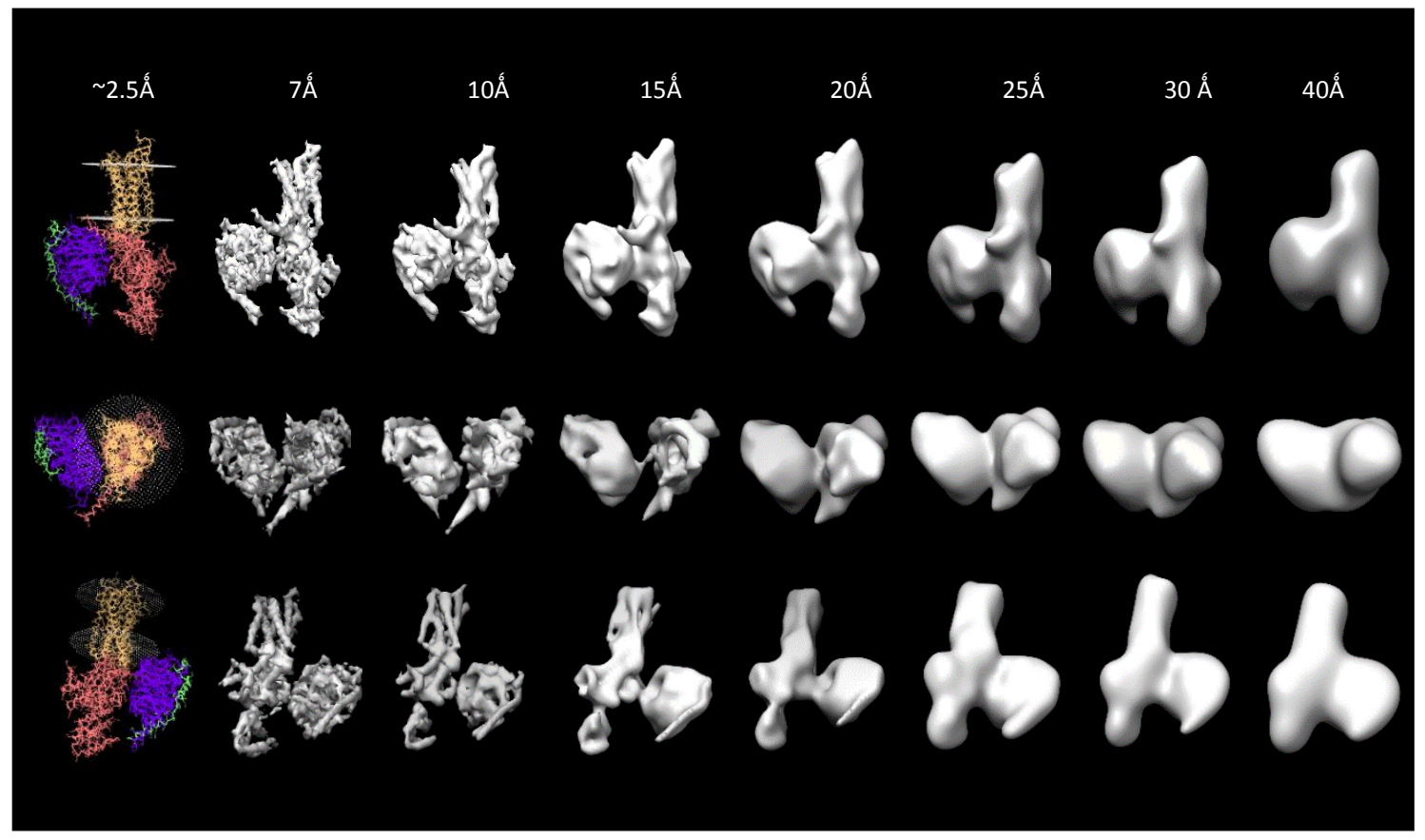

Figure 2.1. Surface maps of an A2aAR-Gs model at various resolutions. The boundaries of the membrane bilayer shown as white discs. The second row shows the original model with a vertical rotation of $90^{\circ}$ of the extracellular region towards the plane of the page relative to the initial image. Row 3 shows the original model with a $180^{\circ}$ horizontal rotation and $45^{\circ}$ tilt of the $\mathrm{EC}$ region away from the page relative to the initial image. 
was then processed via EMAN and Chimera to calculate surface maps at the noted resolutions. The results obtained from these in silico experiments are not directly comparable to actual 3D reconstructions in terms of resolution, as the input data in the former case are ideal. Additionally, the complex is approximately $130 \mathrm{kDa}$, which is close to the theoretical limit $(\sim 110 \mathrm{kDa})$ for single particle analysis, so a high signal to noise ratio is expected. In this case, negative-staining is preferable to cryo methods, as it provides for much stronger contrast [174].

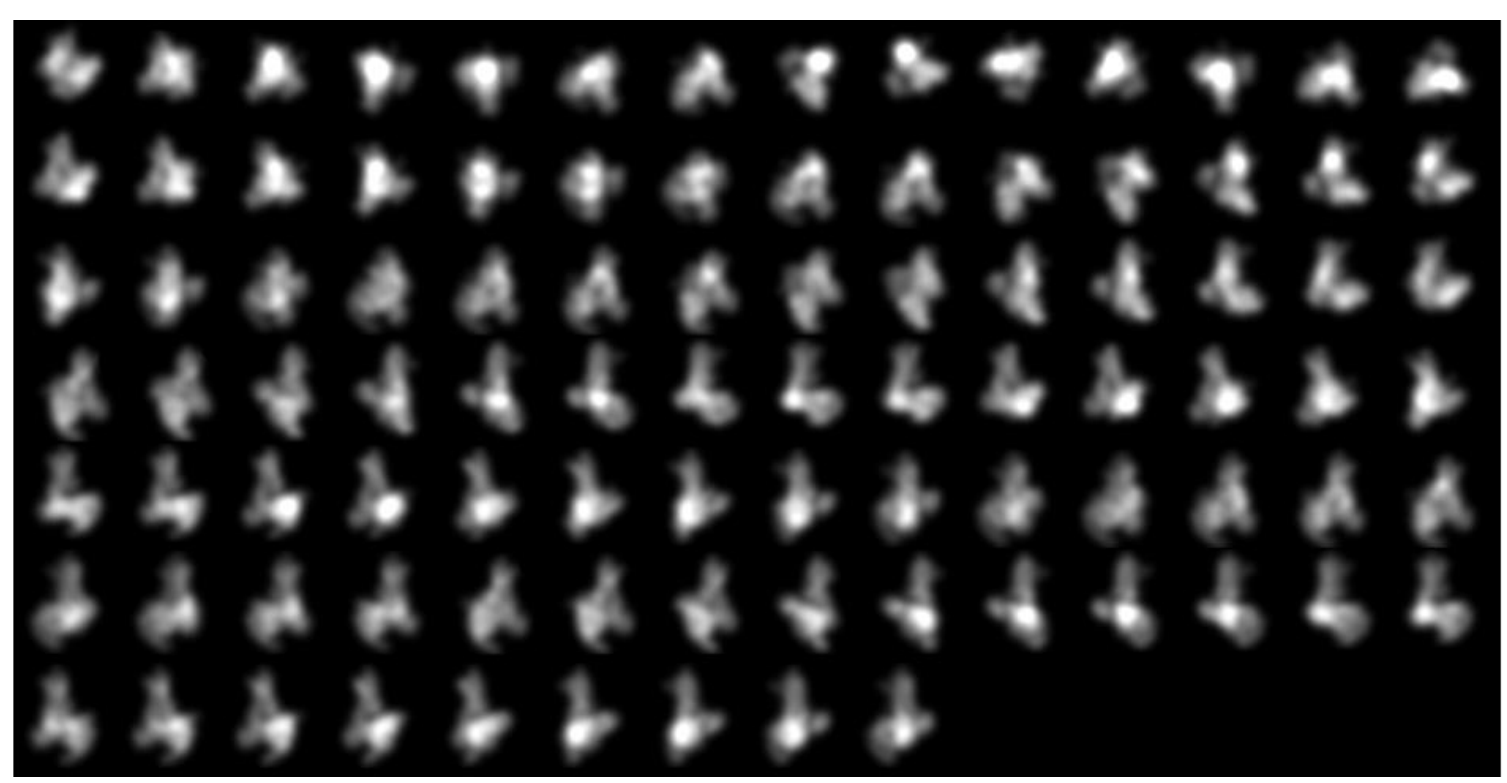

Figure 2.2 Calculated 2D projections of an $A_{2 A} A R-G s$ model. Calculated projections from an $A_{2 A} A R-G s$ model based on the crystal structure of the $\beta_{2} A R-G s, 20 \AA$ resolution at $15^{\circ}$ increments.

Particle arrays were also generated for alternative structures to enhance visual recognition during screening and analysis of micrographs, including a model built incorporating $\mathrm{G} \alpha$ changes seen in the crystal structure of $\beta_{2} \mathrm{AR}-\mathrm{Gs}$, as seen in Figure 2.2, and dimeric complexes of both models (data not shown), as well as $\mathrm{A}_{2 \mathrm{~A}} \mathrm{AR}-\mathrm{G} \alpha_{\mathrm{s}}$ alone (data not shown). Dimeric complexes resulted in a " $\pi$ "-shaped particle and were not frequently 
observed. Figure 2.2 shows arrays of particle projections calculated in silico at a $20 \AA$ resolution at $15^{\circ}$ increments.

\section{Initial Screening}

Multiple negatively-stained constructs were screened by electron microscopy and analyzed by EMAN2, a software suite comprising a set of image processing programs for single particle analysis [166]. Construct screening informed purification as well as complex formation and other laboratory studies where examination of protein heterogeneity and solubility was of interest. Initial micrographs of $\mathrm{A}_{2 \mathrm{~A}} \mathrm{AR}-\mathrm{Gs}$ complexes bore a strong resemblance to rhodopsin-transducin complexes, as seen in Figure 2.3. However, the morphology of the complex was very rounded and not as predicted by in silico experiments.
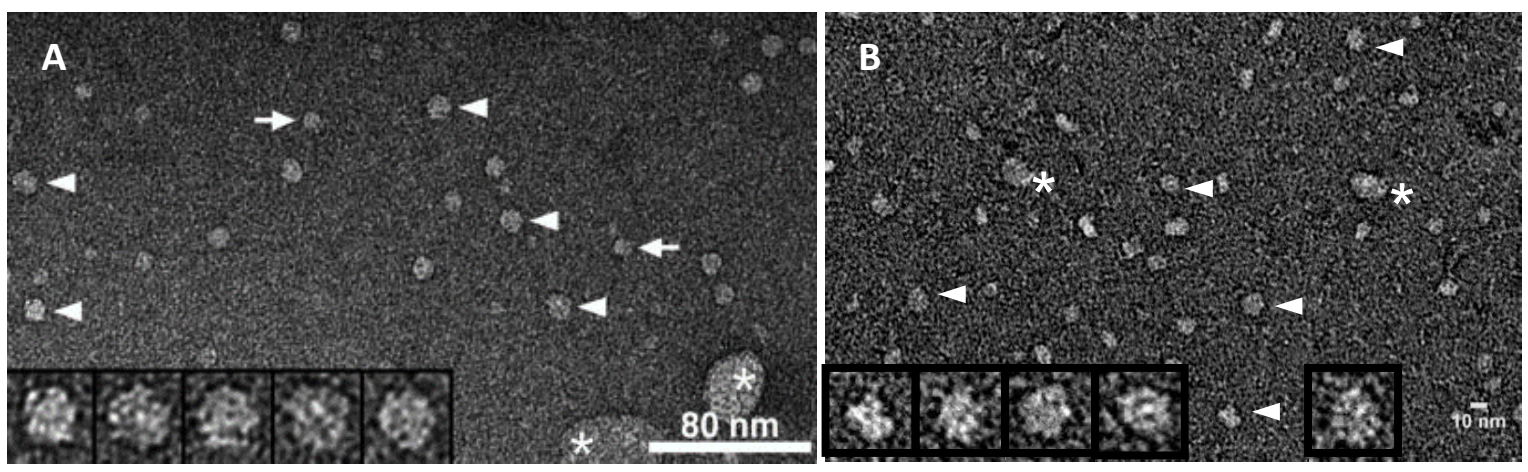

Figure 2.3. Electron microscopy of GPCR-G-protein complexes. A, rhodopsin-transducin [Jastrzebska 2009]. B, $A_{2 A} A R G \alpha_{s}-\beta \gamma$ complexes. Arrows indicate selected particles (boxed). Asterisks indicate large vesicles or aggregates.

\section{D Reconstructions of Adenosine Receptor-G-protein Complexes}

Particles of $\mathrm{A}_{2 \mathrm{~A}} \mathrm{G} \alpha_{\mathrm{s}}-\beta \gamma$ were prepared with three ligands, the $\mathrm{A}_{2 \mathrm{~A}} \mathrm{AR}$ antagonist, caffeine, and the agonists UK-432097 and 2-chloroadenosine. All three reconstructions share similar size, and general perimeter, as seen in Figure 2.4, row 2, and were determined to have a resolution of 23-25 A. In estimating the resolution, the particles are divided into 
an even set and an odd set at random, and used to calculate separate solutions; where the solutions diverge is the resolution limit, accepted as $50 \%$ of the Fourier shell correlation (FSC). The FSC50 curves (Figure 2.4, row 3) are a result of an even odd test. For the three structures, the resolutions are $25 \AA, 23 \AA$, and $23 \AA$, respectively. However, for the caffeine

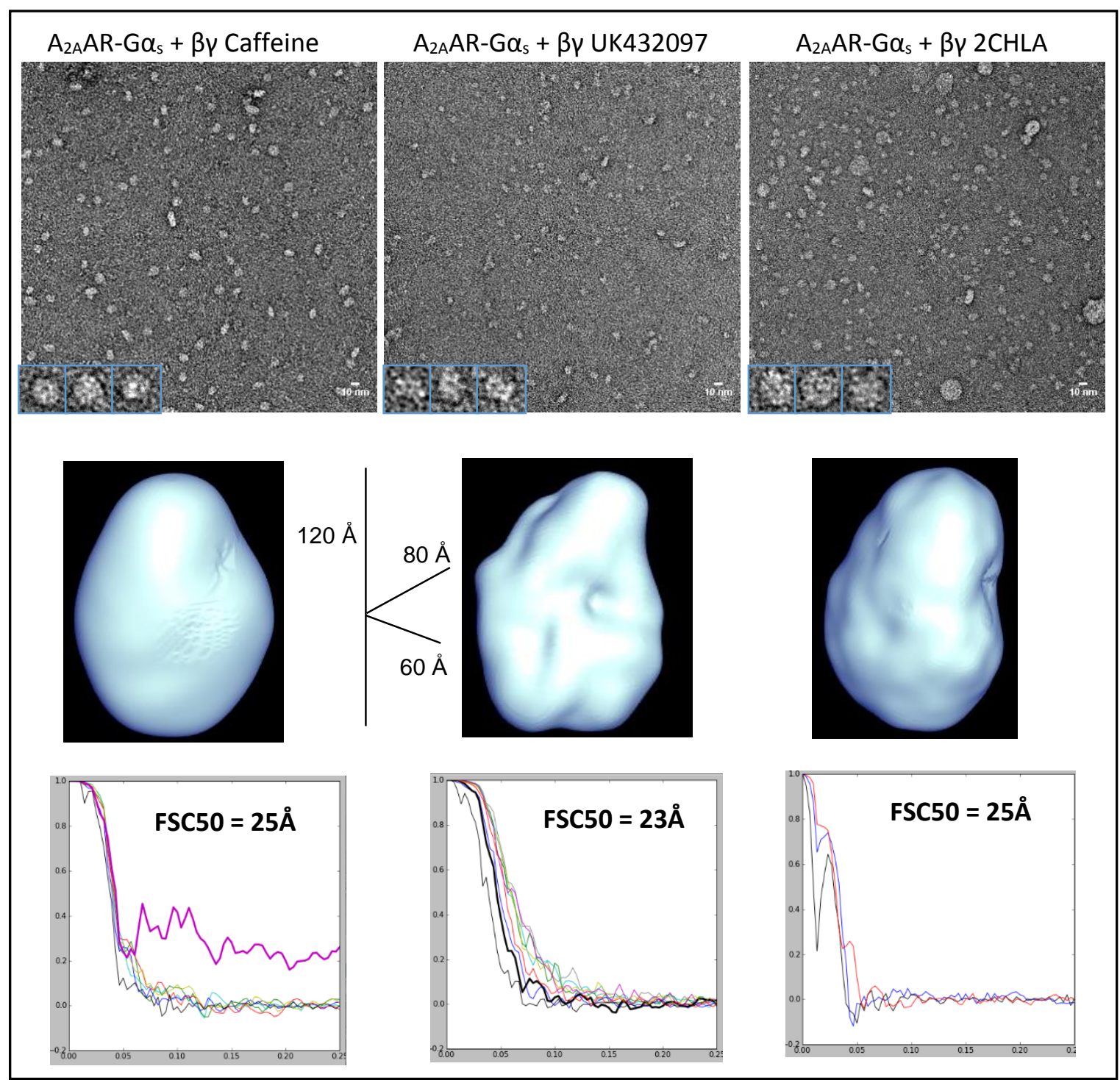

Figure 2.4. 3D reconstruction of $A_{2 A} A R G \alpha_{s}-\beta \gamma$ complexes with selected ligands. Top row, representative micrographs showing negatively stained ARGP complexes. Middle row, surface renderings of $3 \mathrm{D}$ reconstructions. Bottom row, $\mathrm{FSC} 50$ curves for resolution calculation. 
treated sample, the curve did not return to baseline, suggesting a possible problem with the model. The curve for the UK432097-treated sample is ideal.

I speculated that the detergent micelle was contributing to the rounded appearance.

By extensive washing of the grid prior to negative staining, and washing with Biobeads (Bio-Rad), I determined that it was possible to remove additional detergent. Indeed, samples which had been washed more extensively showed more detailed features (data not shown). Particles that had been eluted with a lower concentration of detergent were also

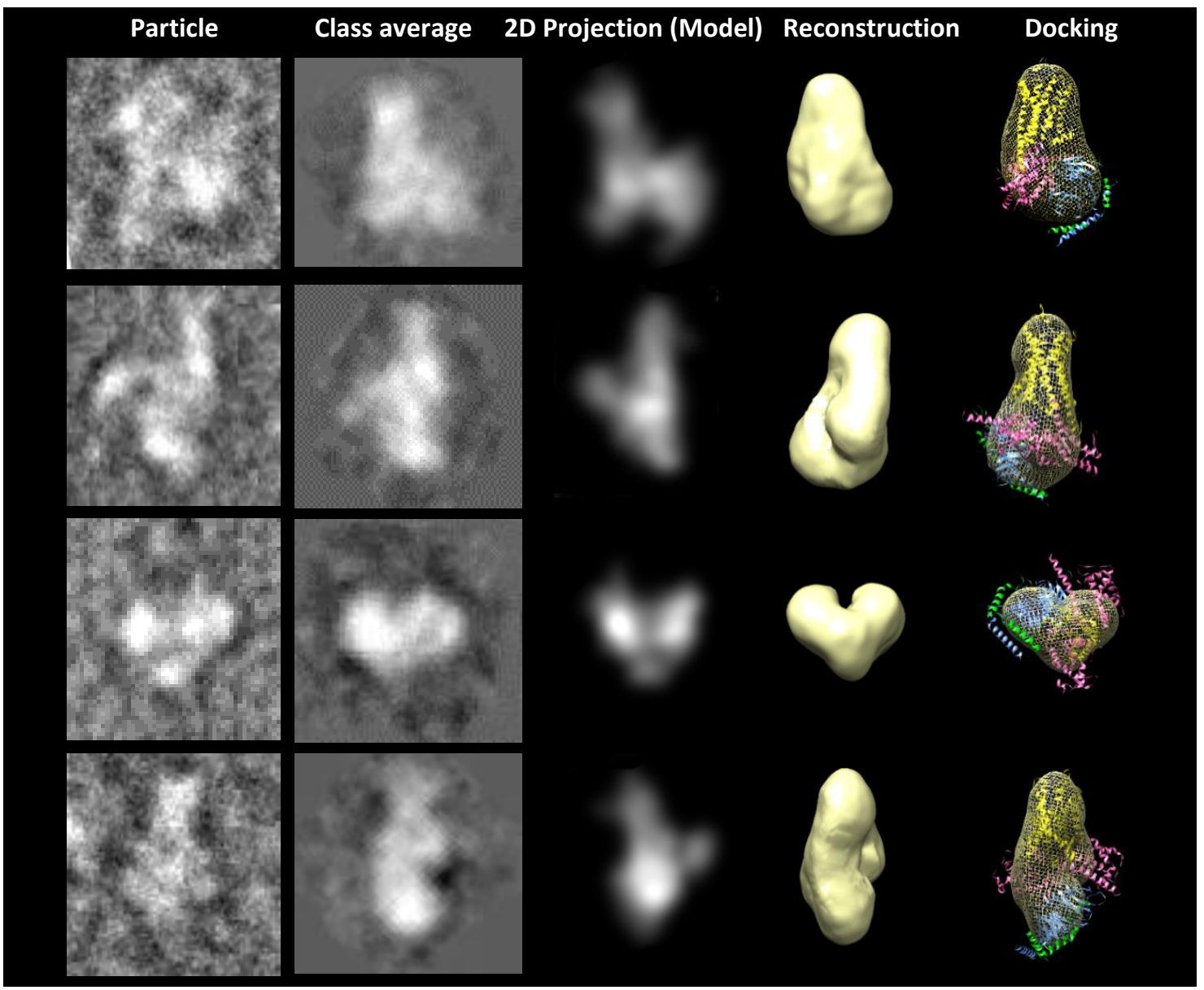

Figure 2.5. 3D reconstruction of an $A_{2 A} G \alpha_{s}-\beta \gamma$ complex. First column, representative particles. Second column, selected class averages. Third column, selected calculated projections. Fourth column, surface rendering of 3D reconstruction. Fifth column, mesh representation of 3D reconstruction fit with $A_{2 A} G s$ model based on the crystal structure of $\beta_{2} A R-G s$, with receptor in yellow, $G \alpha$ in pink, $G \beta$ in blue, and $G \gamma$ in green. 
improved, more so than washing alone (data not shown). Lowering the detergent concentration to $0.025 \%$ DDM had a significant impact on the screening results and 3D reconstructions as seen in Figure 2.5.

The $3 \mathrm{D}$ reconstruction of the $\mathrm{A}_{2 \mathrm{~A}} \mathrm{AR}-\mathrm{Gs}$ reveals a single density for the receptor, with two secondary lobes, the larger of which, as guided by the calculated 2D projections, is assigned to $\mathrm{G} \beta \gamma$, and a smaller lobe of density assigned to $\mathrm{G} \alpha$. Unlike the $\beta_{2} \mathrm{AR}-\mathrm{Gs}$, $\mathrm{A}_{2 \mathrm{~A}} \mathrm{G} \alpha_{\mathrm{s}}-\beta \gamma$ particles do not show preferred orientation on the grid surface. Similar to $\beta_{2} \mathrm{AR}-$ Gs [7], $\mathrm{G} \alpha$ is present, however, there is less density present than the model would predict, suggesting that there is flexibility in the $\mathrm{G} \alpha \mathrm{H}$ domain. In comparison to the $3 \mathrm{D}$ reconstruction of the $\beta_{2} \mathrm{AR}-\mathrm{Gs}, \mathrm{G} \beta \gamma$ is more tightly associated with the complex, and did not require manual fitting to adjust the crystal structure to the reconstruction. In single particle analysis of the adenosine receptor $\mathrm{G}_{\mathrm{s}}$ complex, we did not have the benefit of preferred orientation; thus, it was not possible to achieve the same resolution and make detailed observations about $\mathrm{G} \alpha$ as with the $\beta_{2} \mathrm{AR}$-Gs complex. Critically, however, there is neither a large micelle surrounding the receptor, nor does the receptor appear to be dimeric under the screening conditions. A 6000 particle data set was used to generate the reconstruction. Particles were boxed by size. The resolution was calculated to be $29 \AA$ by FSC50. 


\section{Fiducial Screening}

Because at the time the $\beta_{2} \mathrm{AR}-\mathrm{Gs}$ crystal structure of the complex had not yet been published, we had uncertainty as to how to dock the G-protein to the receptor and conclusively assigning the different lobes of density. In order to address these issues, I generated a panel of Fab fragments from a set of commercially available antibodies directed against $\mathrm{G} \alpha, \mathrm{G} \beta, \mathrm{G} \gamma$, and the $\mathrm{A}_{2 \mathrm{~A}} \mathrm{AR}$, in order to add additional density and to

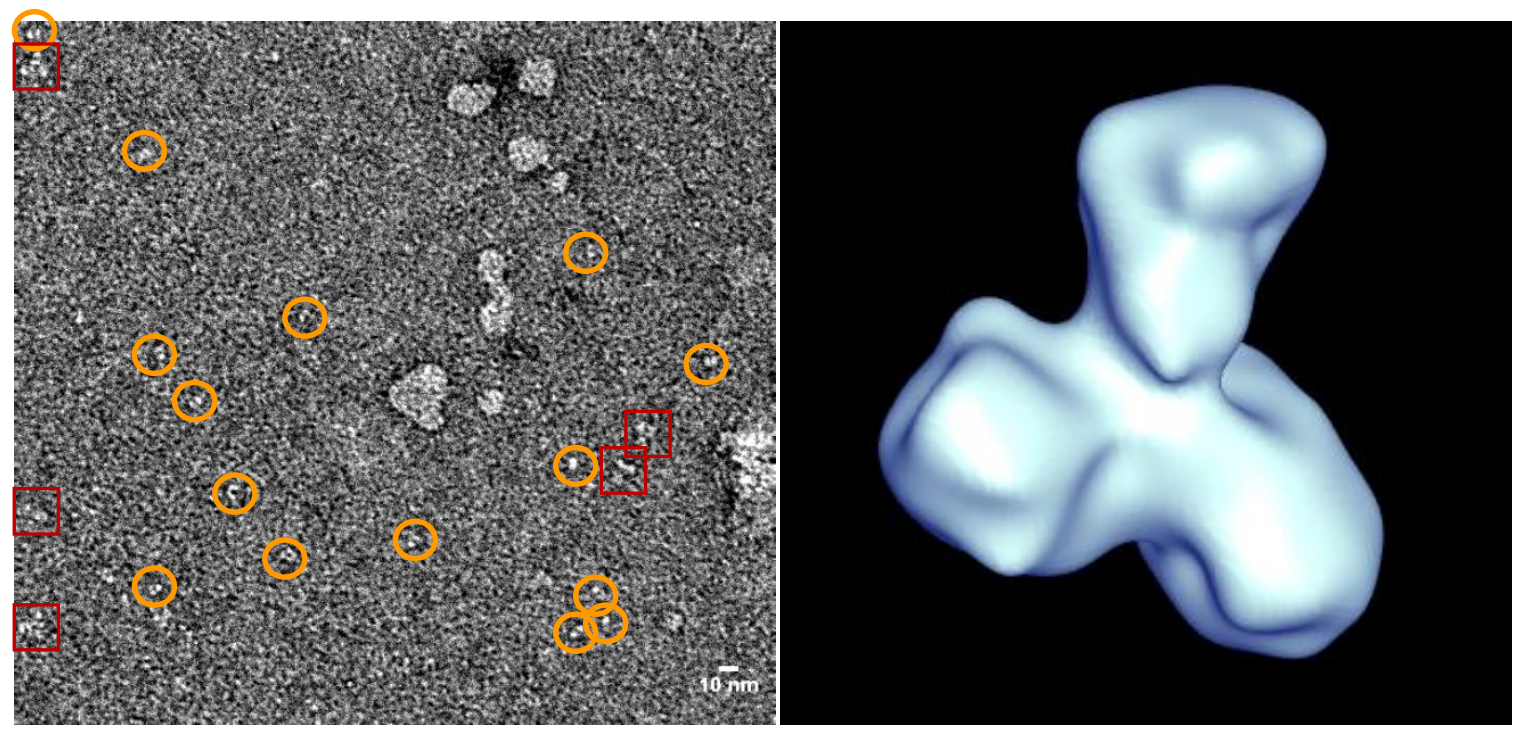

Figure 2.6. Fab screening. Left, representative micrograph showing purified anti-FLAG fab fragments (orange circle), contaminated with whole IgG (red squares). Right, initial model during $3 D$ reconstruction of anti-FLAG fab-bound ARGP complexes. The model is clearly strongly biased by low levels of contaminating IgG.

conclusively identify the different regions of density via generation of difference maps. Of the six different Fabs purified only one anti-G $\beta$, one anti-G $\gamma$, and the anti-FLAG Fabs were of sufficient yield to proceed with (data not shown). Of those, the anti-FLAG Fabs had the best yield. While I was able to generate and purify the Fab fragments (see Figure 2.6), a low level contamination of whole $\mathrm{IgG}$ complicated image processing. Because the whole IgG molecule is approximately the same size as the complex, it was difficult to exclude it during particle picking, despite it being present at very low levels. Because of the internal 
symmetry and strong asymmetric features, the whole IgG strongly biased the first initial model generated as seen in Figure 2.6. More reasonable initial models were generated as well, however, these simple bipartite structures were not informative (data not shown). Attempts to further purify the Fab-bound complex failed due to an insufficient quantity of antibody necessary for preparative SEC (data not shown).

In addition to Fabs, nitrilotriacetic acid (NTA) conjugated gold particles have also been useful in identifying particular domains, or as fiducials, in electron microscopy [175]. The nanogold (Nanoprobes), is capable of binding histidine tags, present on some $\mathrm{A}_{2 \mathrm{~A}}$ adenosine receptor constructs ( $\mathrm{T} 4 \mathrm{~L}-\mathrm{A}_{2 \mathrm{~A}} \mathrm{AR}$ ). While this method proved to be potentially useful for connexin and ZnT8 studies (data not shown), it was not feasible for single particle analysis of the $\mathrm{A}_{2 \mathrm{~A}} \mathrm{AR}-\mathrm{Gs}$. Nanogold-labeling was informative however for general electron microscopy studies of the adenosine receptor. When an Nb35-stabilized T4L-A $\mathrm{A}_{2 \mathrm{AR}} \mathrm{AR}$-Gs complex was decorated with $5 \mathrm{~nm}$ nanogold and washed $6 \mathrm{X}$, a high degree of decoration was observed, as seen in Figure 2.7, left lower panel.

Nanogold-labeled complexes had a "fried-egg" appearance. Strong contrast due to the gold particles obscures visual recognition complex features, as well as the orientation of the gold particle on top of the complex. The nanogold was thus not a useful fiducial for 3D reconstruction of the complex. When the Nb35-stabilized T4L- $\mathrm{A}_{2 \mathrm{~A}} \mathrm{AR}-\mathrm{Gs}$ complex was incubated with $100 \mathrm{um} \mathrm{GTP} \gamma \mathrm{S}, \mathrm{A}_{2 \mathrm{~A}} \mathrm{AR}$ dimers were observable along with other complex components, as seen in Figure 2.7, top right panel. 

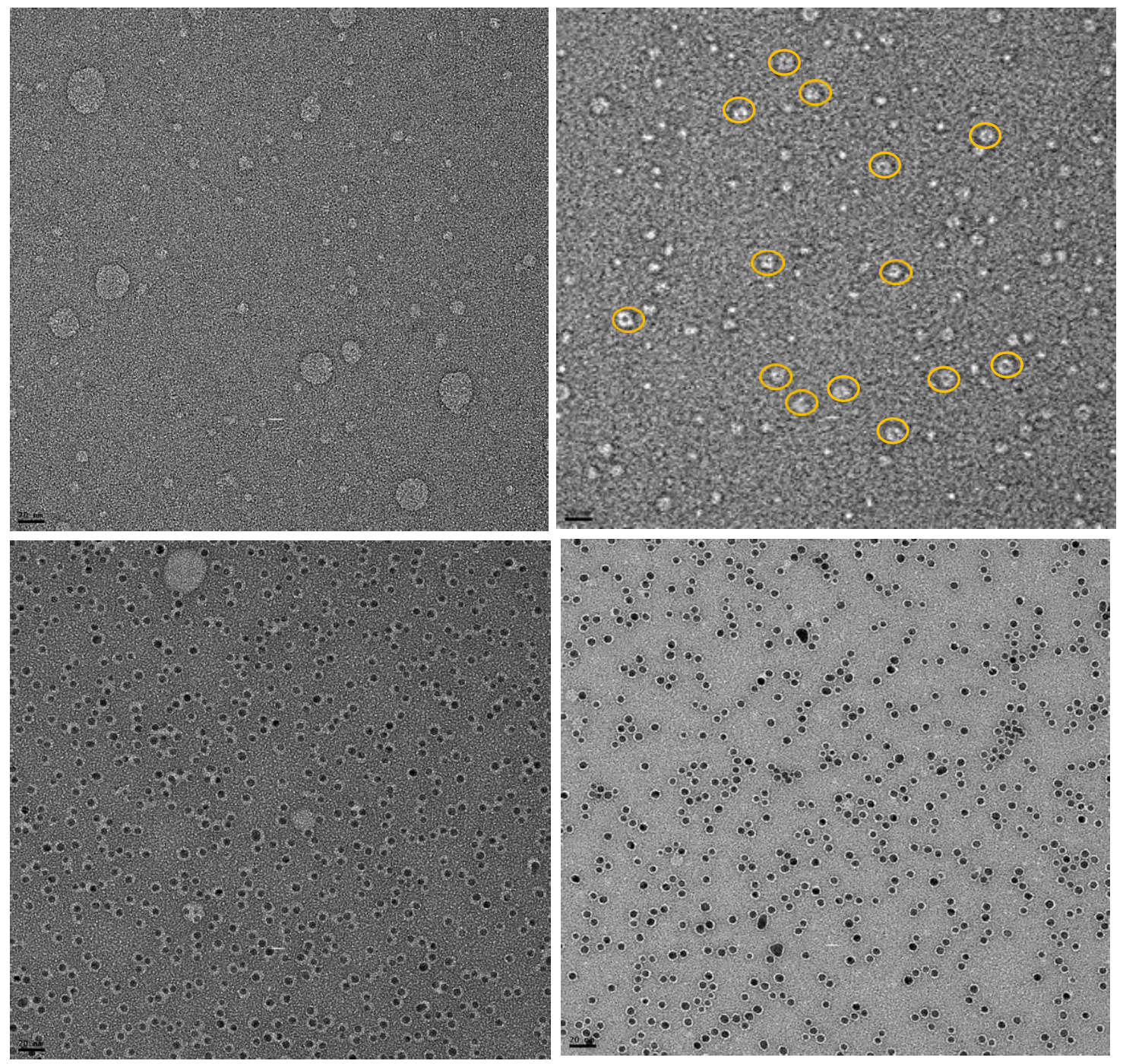

Figure 2.7. Nanogold binding of T4L-A $\mathrm{A}_{2 \mathrm{~A}}-\mathrm{Gs}$ complexes. Top left, untreated Nb35-stabilized nucleotide-free T4L-A $\mathrm{A}_{2 \mathrm{~A}} \mathrm{Gs}$ complexes. Top right, GTP $\gamma \mathrm{S}$-treated Nb35-stabilized complex showing complex dissociation and receptor dimers (orange circles). Bottom left, nanogoldlabeled Nb35-stabilized T4L-A $2 A$ AR-Gs complexes. Bottom right, nanogold-bound dissociated $T 4 L-A_{2 A}$ shows no features.

\section{D Reconstructions of $A_{1} A R$ and $A_{2 A} A R$}

The publication of the crystal structure of the $\beta_{2} \mathrm{AR}-\mathrm{Gs}$ complex [7] provided the key for alignment of the G-protein to the $\beta_{2} \mathrm{AR}$, however, combined with the publication of the rhodopsin-transducin complex [5], there is some question as to the stoichiometric composition of the density that has been assigned to the receptor in the respective instances. 
Previous $\mathrm{A}_{2 \mathrm{~A}} \mathrm{AR}-\mathrm{Gs} 3 \mathrm{D}$ reconstructions are fit well to a single receptor without a large micelle, unlike the $\beta_{2}$ AR-Gs and rhodopsin-transducin complex. To confirm the fitting of the receptor in the molecular envelope of the $3 \mathrm{D}$ reconstruction, I examined two other constructs. By reducing the complexity of the target, I speculated that the results may be more interpretable. Two constructs were utilized, $A_{1} A R-G \alpha_{i}$ and T4L-A $2 A$ AR. Both receptor constructs were negatively-stained and subjected to single particle analysis and $3 \mathrm{D}$ reconstruction in EMAN2.

A model of the $A_{1} A R$ based on the structure of T4L-A $A_{2 A} A R$ was manually docked in the asymmetric hourglass-shaped density without any modifications as seen in Figure 2.8. Although possible, it is unlikely that the receptor fit is incorrect with respect to its long axis, because the density is highly asymmetric, with a narrowing at the center. There is no density for $G \alpha_{i}$, suggesting that the linker was highly flexible and that there was no coupling under the experimental conditions. The asymmetric features along with the size of the envelope also preclude good docking for a receptor dimer. The size of the density is

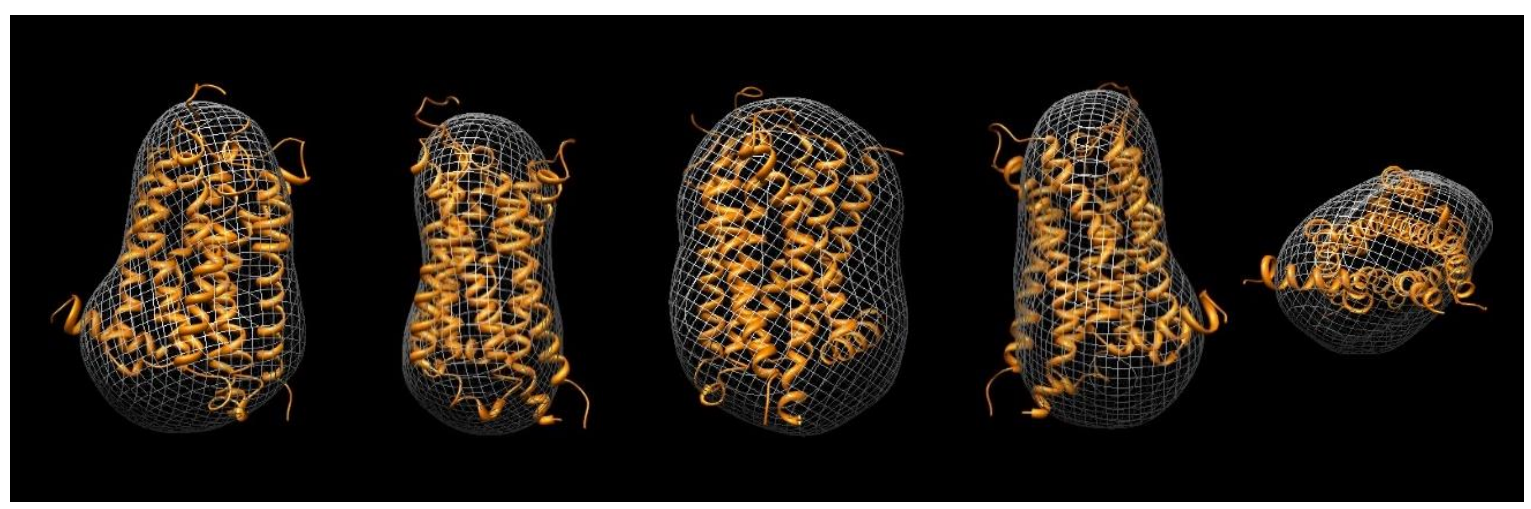

Figure 2.8. 3D reconstruction of adenosine receptor, $A_{1} A R_{s}$. Reconstruction represented by mesh. A model of the $A_{1} A R$ (orange) was manually docked into the molecular envelope. Docked reconstructions are related by clockwise rotations of the long axis of the receptor, with the rightmost image showing the intracellular face of the receptor, aligned as with the first image. 
consistent with the expected size of a single monomeric receptor. The detergent, DDM, did not appear to create a large micelle under the conditions used, which included an extensive wash of the grid prior to negative staining. $32 \AA$ resolution was achieved with a 3400

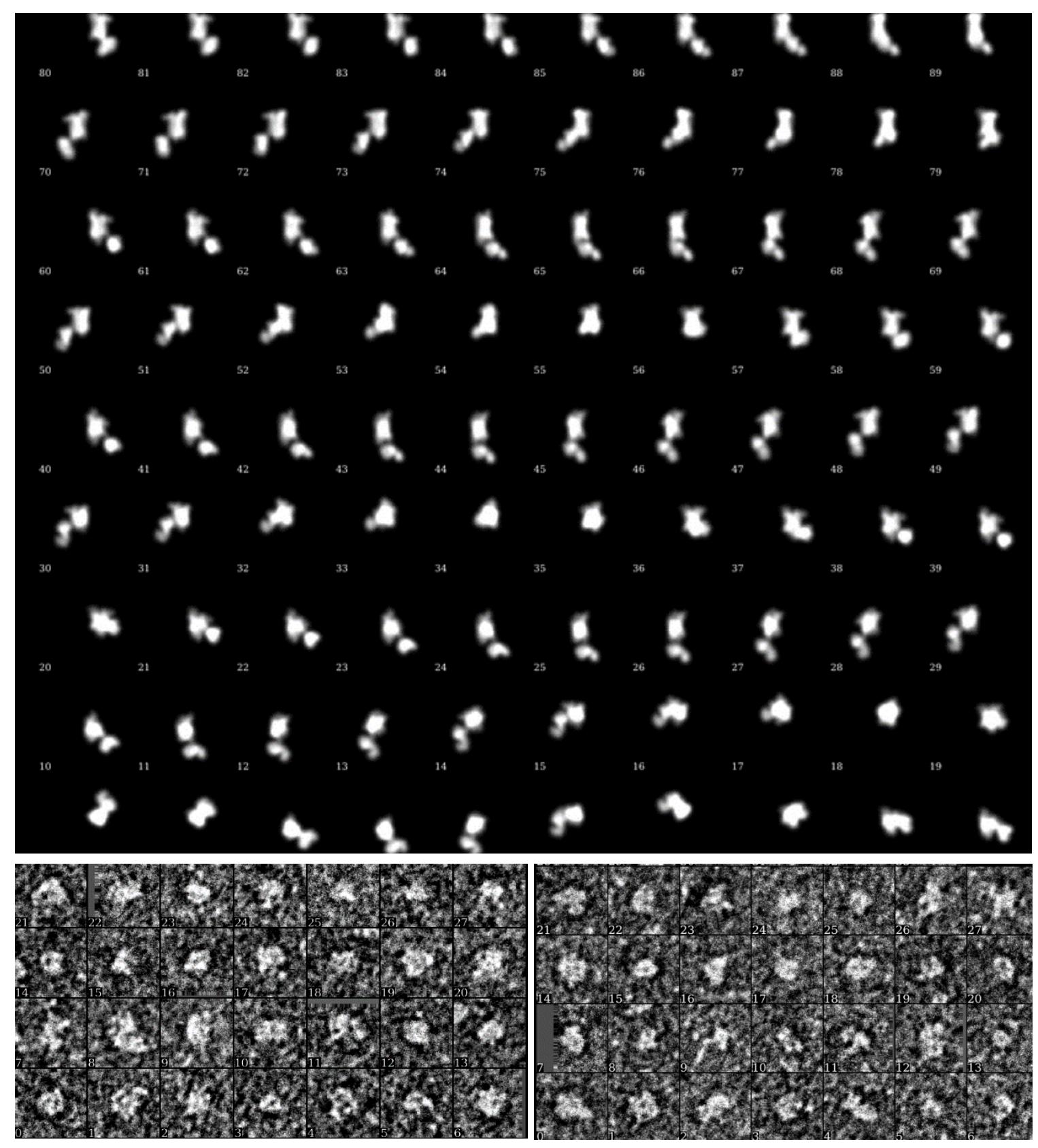

Figure 2.9. Two-dimensional projection analysis of T4L-A $A_{2 A}$. Top, calculated $2 \mathrm{D}$ projection images using EMAN2 at $20 \AA$ resolution in $15^{\circ}$ increments. Bottom, boxed particles from a representative micrograph of DDM-solubilized T4L- $A_{2 A}$ (left) and LMNG-solubilized T4L-A $A_{2 A}$ (right). 
particle set, despite the small size of the receptor. The intracellular view suggests that there may be small differences in the orientations of the helices, however the resolution is too low to draw more specific conclusions.

As an additional reduced-construct control, and to examine whether detergent effects may be playing a role in the shape of the receptor density, I examined the T4L$\mathrm{A}_{2 \mathrm{~A}} \mathrm{AR}$ in the two detergents utilized in the other single particle studies, DDM and LMNG. Figure 2.9 shows boxed particles from a representative image from $0.025 \%$ DDMsolubilized (left) and $0.025 \%$ LMNG-solubilized (right) T4L-A $2 \mathrm{~A} A$ R.

While similar, the LMNG-solubilized particles bore more similarity to calculated 2D model projections, and bipartite densities were more evident in LMNG-solubilized particles. Highly elongated particles were however not evident in either sample, suggesting that T4L is oriented differently with regards to the receptor than seen in the crystal structure of the T4L- $\beta 2 \mathrm{AR}, 3 \mathrm{SN} 6[6]$.

T4L-A $2 A$ AR particles were highly asymmetric, and had slight hourglass contours, as seen in Figure 2.10. Fitting was straightforward; however, there was less density seen for T4L than expected. It is possible that there is flexibility in the linker between the receptor and T4L. In this case, only the core density would be represented and portions further afield would be lost to averaging. Again, there is little density surrounding the receptor that is unaccounted for and it appears that the receptor is monomeric. 


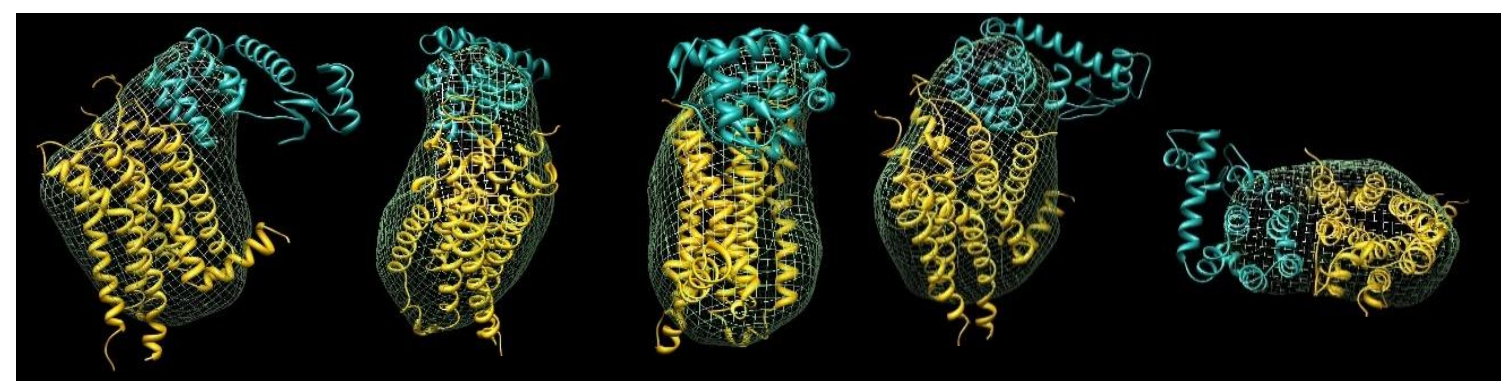

Figure 2.10. Three-dimensional reconstruction of T4L-A2AAR. Reconstructed volume represented by mesh, with $A_{2 A} A R$ manually docked in yellow, and T4L docked in cyan.

\section{Discussion}

Single particle analysis of GPCRs and G-protein complexes, in conjunction with parallel studies such as crystallography, has aided in the discovery of the mechanism for nucleotide release by $\mathrm{G} \alpha$; however, it has prompted the need for further examination of the receptor stoichiometry in the GPCR-G-protein complex. In the case of the adenosine receptor, the results presented here strongly suggest that the adenosine receptors $A_{1}$ and $\mathrm{A}_{2 \mathrm{~A}}$ exist as monomers when detergent-solubilized, and that within the detergentsolubilized $\mathrm{A}_{2 \mathrm{~A}}-\mathrm{Gs}$ complex, the adenosine receptor is bound to the G-protein at a ratio of 1:1. Because the constructs used contained modifications at the N-termini, it cannot be ruled out that steric clashes there due to the presence of tags and $\mathrm{N}$-terminal fusions may have been disruptive to dimer formation.

Examination of GTP $\gamma \mathrm{S}$-treated complexes revealed dissolution of the complex, as well as an unexpected finding: dimerization of the receptor and burial of the $\mathrm{N}$-terminal histidine tag. Given the unavailability of the tag for nanogold binding, the results suggest dimer formation may be occurring at the H8-H1 interface. Artifacts resulting from association through the His-tag are unlikely as an imidazole wash was performed prior to 
staining. Although head to tail dimer formation cannot be ruled out, this finding offers an alternative interpretation for the bi-lobed density seen in the single particle analyses of the $\beta_{2} \mathrm{AR}$; Figure 2.11, left panel. The authors asserted that because only one density was observed for T4L, that the receptor must be monomeric. Dimer formation at the H8-H1 interface would bring the respective T4Ls into proximity, and at the low resolution observed, may appear as one density.

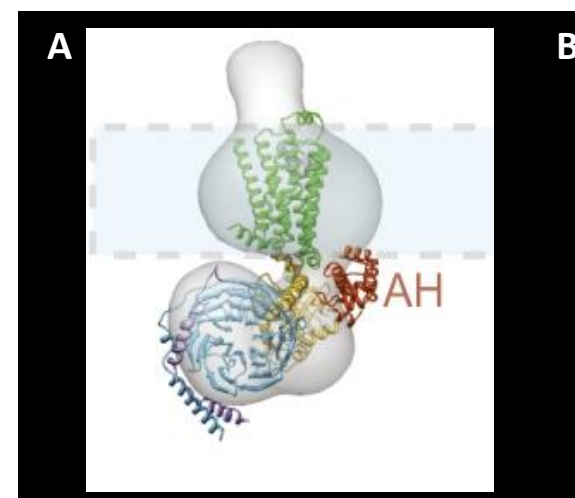

B2AR-Gs

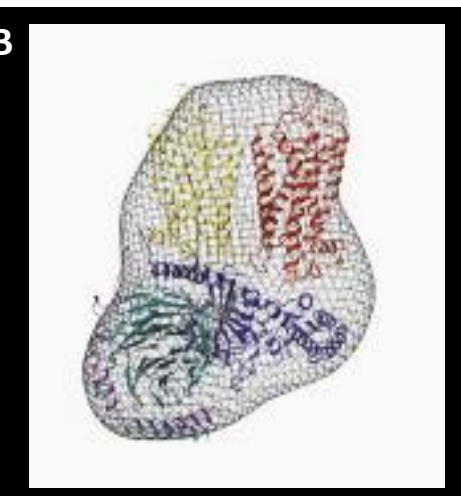

Rho-G

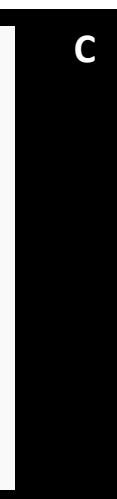

C

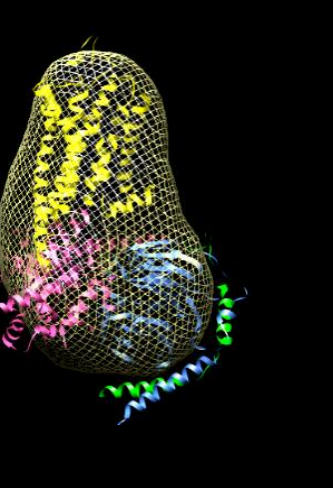

$A 2 a G \alpha_{s}-\beta \gamma$

Figure 2.11. Single particle 3D reconstructions of three GPCR-G-protein complexes. $A, \beta_{2} A R-$ Gs. Figure from: PNAS, Vol. 108(38): p. 16086-91, Westfield, G., Rasmussen SG, Su M, Dutta S, DeVree BT, Chung KY, Calinski D, Velez-Ruiz G, Oleskie AN, Pardon E, Chae PS, Liu T, Li S, Woods VL, Steyaert J, Kobilka BK, Sunahara RK, and S. G., Structural flexibility of the G alpha s alphahelical domain in the beta2-adrenoceptor Gs complex, Copyright 2011 with permission. B, Rhodopsin-transducin, reprinted from: the Journal of Structural Biology, Vol. 176(3):387-94, Jastrzebska, B., P. Ringler, D.T. Lodowski, V. Moiseenkova-Bell, M. Golczak, S.A. Muller, K. Palczewski, and A. Engel, Rhodopsin-transducin heteropentamer: three-dimensional structure and biochemical characterization, copyright 2011, with permission from Elsevier. $C, A_{2 A} G \alpha_{s^{-}}$ $\beta \gamma$.

User bias can be a significant issue in single particle analysis. Because it is possible to hand-select individual particles, bias can be introduced into a data set. By selecting only particles which resemble expected solutions or models, one may be analyzing only a subpopulation of particles. Caution must be used when attributing to a larger population the condition of a particular state. To avoid these issues, I selected all particles of the 
expected size, without respect to their resemblance to the $2 \mathrm{D}$ projections. By including all data, multiple subpopulations may be revealed in the two-dimensional class averages.

Directed protein engineering has been useful in generating highly crystallizable GPCR constructs and may be of use in the case of single particle analysis as well in creating features which facilitate identification in EM analysis. Insertion of a histidine tag in the third extracellular loop of the receptor, in conjunction with nanogold binding, may provide an additional orienting fiducial. Further method development in chemical coupling to the grid, similar to what has been done with biosensor chips, would be useful towards directed particle orientation and beneficial to the field. Some progress has already been made in this direction, such as the Ni-affinity grid developed by the Kelly lab [176].

This work describes the earliest known single particle structure of a GPCR-Gprotein complex. Although three-dimensional reconstruction could only reveal crude features of the complex, taken with the three-dimensional reconstructions of the $A_{1} A R$ and $\mathrm{A}_{2 \mathrm{~A} A R}$, the receptor density can clearly be attributed to a monomeric receptor. It is only with additional GPCR-G-protein complex structures that the debate regarding receptor-Gprotein stoichiometry will be resolved. If binding and G-protein activation provokes conformational changes in the receptor which affect its propensity to form dimers, then we must be more cautious in interpretation. It is now no longer sufficient to ask whether GPCRs exist and act as monomers or dimers, but under what conditions receptor dimers form and how dimerization affects receptor activity. 


\section{Experimental Methods}

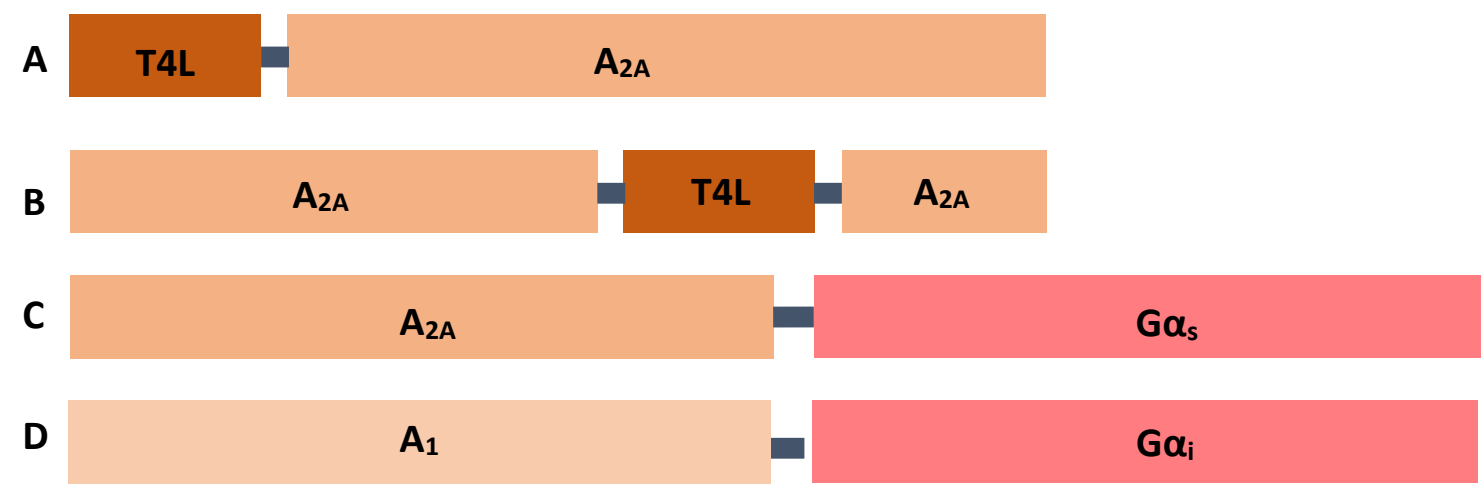

Figure 2.12. Constructs used in this work. $A$, an N-terminal T4 lysozyme- $A_{2 A}$ adenosine receptor fusion. $B$, an $A_{2 A}$ receptor construct with $T 4 L$ in place of the third intracellular loop. $C$, an $A_{2 A}$ adenosine receptor with a $C$-terminal $G \alpha_{i}$ fusion. $D$, an $A_{1}$ adenosine receptor with a C-terminal $G \alpha_{i}$ fusion.

\section{Construct design and purification}

Multiple $\mathrm{A}_{2 \mathrm{~A} A R} \mathrm{~A}$ constructs were examined during the course of this work, and were designed by Susan Leonhardt. All constructs unless otherwise stated were expressed by baculovirus expression in S. frugiperda. Schematics representing the used constructs are shown in Figure 2.12.

The initial construct is an $\mathrm{A}_{2 \mathrm{~A}} \mathrm{G} \alpha_{\mathrm{s}}$ fusion, described elsewhere [177]. Complexes of $A_{2 A} G \alpha_{s}-\beta \gamma$ were purified from pellets derived from triple infection of $A_{2 A} G \alpha_{s}, G \beta_{4}$, and $\mathrm{G} \gamma_{2}$. A $1.5 \mathrm{~L}$ culture provided approximately a $30 \mathrm{~g}$ cell pellet. Cell pellets were homogenized in $120 \mathrm{ml} 10 \mathrm{mM}$ HEPES pH 7.5, $10 \mathrm{mM} \mathrm{KCl}$, and $10 \mathrm{mM} \mathrm{MgCl}_{2}$, with EDTA-free protease inhibitor tablet (Roche) using a microfluidizer at 15 psi and spun at 36,000 rpm in a Ti45 to collect the membranes. The membranes were then dounce homogenized on ice with $30 \mathrm{ml}$ of $20 \mathrm{mM}$ HEPES pH 7.5, $20 \mathrm{mM} \mathrm{NaCl}, 10 \%$ glycerol and 5 uM GDP with protease inhibitor for 40 strokes. Consequently the membranes were spun 
down and this wash step repeated. Membranes were solubilized by the addition of $50 \mathrm{ml}$ 20 mM HEPES pH 7.5, 20 mM NaCl, 10\% glycerol, 100 uM adenosine, 1 mM EDTA, and $1 \% \mathrm{DDM} / 0.2 \% \mathrm{CHS}$ and incubated at $4^{\circ} \mathrm{C}$ for 3 hours. The solubilized membranes were collected following centrifugation at 36,000 rpm in a Ti45 rotor to pellet insoluble material. The solubilized membranes were added to $2 \mathrm{ml}$ of solubilization buffer-equilibrated antiFLAG resin (Sigma-Aldrich) and incubated overnight with rocking at $4^{\circ}$. Complex-bound beads were pelleted at $3500 \mathrm{rpm}$ on a Beckman tabletop centrifuge, diluted with buffer and applied to a gravity column. Three washes with solubilization buffer removed unbound components. Purified complex was eluted with 4 x $1 \mathrm{ml}$ aliquots $0.2 \mathrm{mg} / \mathrm{ml}$ solution of FLAG peptide in solubilization buffer with 10 minute incubations for each at room temperature. Complex eluted in the first two fractions.

Cell pellets of approximately $20 \mathrm{~g}$ containing T4L- $\mathrm{A}_{2 \mathrm{~A}} \mathrm{AR}$ were resuspended in 10 mM HEPES pH 7.5, $20 \mathrm{mM} \mathrm{KCl}$, and $10 \mathrm{mM} \mathrm{MgCl}_{2}$ and dounce homogenized using 40 strokes on ice and centrifuged. Two additional washes with this buffer were performed with centrifugation at $36,000 \mathrm{rpm}$ in a Ti45 rotor. The same buffer containing $800 \mathrm{mM}$ $\mathrm{NaCl}$ was then used to wash the pellets twice more. Pellets were resuspended in $50 \mathrm{ml}$ of $50 \mathrm{mM}$ HEPES pH 7.5, $800 \mathrm{mM} \mathrm{NaCl}, 10 \mathrm{mM} \mathrm{MgCl}_{2}, 10 \%$ glycerol, $2 \mathrm{mg} / \mathrm{ml}$ iodoacetamide, $4 \mathrm{mM}$ theophylline and an EDTA-free protease inhibitor tablet (Roche) and incubated with rocking for 30 minutes at $4^{\circ} \mathrm{C}$ to induce dissociation of any native Gproteins. Following this, membranes were solubilized with $0.5 \%$ DDM/0.1\% CHS for 3 hours with rocking at $4^{\circ} \mathrm{C}$ and centrifuged to remove insoluble material. $2 \mathrm{ml}$ of bufferequilibrated TALON cobalt-affinity resin (Clontech) was added to the solubilized 
membranes and incubated overnight at $4^{\circ} \mathrm{C}$ with rocking. The receptor-bound resin was collected by centrifugation in a Beckman tabletop centrifuge at 1000xg, diluted and applied to a gravity flow column. The receptor was washed twice with $10 \mathrm{ml}$ of buffer $(50 \mathrm{ml}$ of $50 \mathrm{mM}$ HEPES pH 7.5, $800 \mathrm{mM} \mathrm{NaCl}, 10 \mathrm{mM} \mathrm{MgCl}_{2}, 10 \%$ glycerol) with $4 \mathrm{mM}$ theophylline and $8 \mathrm{mM}$ ATP. T4L-A $2 \mathrm{~A} A R$ was eluted with $200 \mathrm{mM}$ imidazole in the buffer containing $0.025 \% \mathrm{DDM} / 0.005 \% \mathrm{CHS}$ (Anatrace/Sigma) and concentrated in a Vivaspin centrifugal concentrator (GE) with a $50 \mathrm{kDa}$ molecular weight cutoff.

Size exclusion chromatography was performed using AKTA with a Superdex 10/300 or Zenix SEC-300 column using a $250 \mu \mathrm{l}$ loop and $125 \mu \mathrm{l}$ sample. The column was equilibrated in $20 \mathrm{mM}$ HEPES pH 7.5, $150 \mathrm{mM} \mathrm{NaCl}, 10 \mathrm{mM} \mathrm{MgCl}_{2}$, and 0.025\% DDM. Alternating peak fractions were analyzed by SDS-PAGE.

$\mathrm{A}_{1} \mathrm{AR}-\mathrm{G} \alpha_{\mathrm{i}}$ was expressed in HEK cells, purified, and provided by Bill McIntire. Briefly, it was purified by affinity tag using TALON resin (Clontech) in DDM/CHS.

\section{Sample preparation and electron microscopy}

For negative-staining, a 300 mesh copper grid with a plastic film and carbon coating was glow discharged to improve the hydrophilicity and sample adherence. $2.5 \mu \mathrm{l}$ of 0.025 $\mu \mathrm{g} / \mu \mathrm{l}$ solutions were applied to glow discharged grids on Parafilm for 2 minutes, and washed by applying to the surface of a $5 \mu l$ water droplet, blotted gently using filter paper (Whatman), placed on another $5 \mu 1$ droplet of water, blotted again, applied to a $5 \mu$ droplet of 0.02 micron filtered $2 \%$ uranyl acetate for 45 seconds, allowed to dry and stored. Where 
noted previously in the chapter, 6 water washes were employed to remove excess detergent. For nano-gold images, washes were done with uranyl acetate to aid in particle fixation.

Specimens were examined at low dose on a Tecnai T12 or Tecnai F20 at $120 \mathrm{kV}$ acceleration voltage at room temperature. Images were typically recorded at approximately $56,000 \mathrm{X}$ magnification using a $2 \mathrm{k}$ or $4 \mathrm{k}$ camera respectively for the two microscopes. Specimens were examined at multiple defocus values in the range of 0.5-1.5 um.

Each receptor and G-protein construct, as well as complex preparations were negatively-stained and screened by EM, constituting the bulk of my data (approximately 10,000 images). Screening was also useful for evaluating the effects of different detegents and buffer changes.

\section{Three-dimensional reconstructions}

Frequently samples were too heterogeneous to process further. Complexes which were not nanobody stabilized were particularly heterogeneous, and were not suitable for processing [Steven Ludtke, written communication]. Image processing was performed in all cases using the EMAN2 suite of programs with CTF correction applied to all images. No preferred orientation was observed. All particles within the expected size range were hand-selected. A likeness filter was applied during model generation keeping $80 \%$ of all particles. For $A_{2 A} G_{\alpha s}-\beta \gamma, 6000$ particles were hand selected for analysis by size. The

resolution of the 3D reconstruction was $29 \AA$. For $\mathrm{A}_{1} \mathrm{AR}, 3400$ particles were selected, with a final resolution of $32 \AA$ for the $3 \mathrm{D}$ reconstruction. For T4L-A2A AR, 8000 particles were 
selected after separate 3D reconstructions for two sets of approximately 4000 particles generated nearly identical solutions with a final resolution of $25 \AA$. Five reconstructions were not included in this chapter, three of which had a low particle number, and three for which the models failed. Those with successful solutions but low particle number are: cross-linked T4L-A2AAR-Gs, 1200 particles, 33 Å reconstruction; non-cross-linked T4LA 2 AAR-Gs (unstabilized), 514 particles $25 \AA$ reconstruction; non-cross-linked T4L-

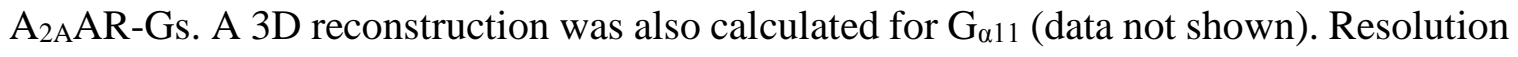
for each reconstruction was made by Fourier shell correlation at 50\%. All models were contoured to a volume consistent with the expected molecular weight of the protein.

\section{Fab preparation and nanogold labeling}

For fab purification, a commercially-available Pierce fab purification kit (Thermoscientific), which utilizes papain digestion to cleave IgG, was used to prepare fabs for binding. Anti-FLAG IgG (SIGMA) as well as number of commercially available (Abcam) IgGs directed against the G-protein were utilized. Desalted IgG was added to digestion buffer-equilibrated papain columns. The digestion reaction was carried out with rocking at room temperature for 6 hours, after which the column centrifuged at 5000xg to collect digested fragments. The column was washed with PBS to collect additional material and pooled. Digestion completion was assessed by SDS-PAGE (data not shown). The digested material was added to a PBS-equilibrated Protein A column to remove the Fc fraction of the $\mathrm{IgG}$ and incubated with rocking for 10 minutes. Flow through and PBS wash containing the fab fragments were collected. Given the limited quantity of IgG and consequently the fab fragments (which have a yield of $<50 \%$, it was not possible to track 
fab-bound complex by SEC. Instead, purified anti-FLAG fabs in PBS were mixed at a 1:1 molar ratio with complex in solution and incubated for 1 hour at room temperature. Negative-staining was performed as described above. Whole anti-FLAG IgG aggregated under buffer conditions necessary to examine the complex (data not shown). An activating IgM antibody, ADONIS [By 2009] was also screened as a possible fiducial, however due to internal flexibility within the arms of the antibody, it was decided that it was unsuitable (data not shown).

For gold-labeling, samples were pre-incubated prior to $5 \mathrm{~nm}$ nanogold decoration. $2.5 \mu \mathrm{l}$ purified complex was applied to glow-discharged grids at $0.025 \mathrm{ug} / \mathrm{ul}$ for 2 minutes. Following incubation, the grid was blotted lightly with filter paper and placed sample-side down on a $5 \mu$ d droplet of $5 \mathrm{~nm}$ nanogold (Nanoprobe) on Parafilm M (Bemis), sealed with a glass petri dish and incubated for 30 minutes at room temperature. Non-specifically bound gold was removed via a wash by inversion over a droplet of $20 \mathrm{mM}$ Tris at $\mathrm{pH} 7.5$, $150 \mathrm{mM} \mathrm{NaCl}$ and $8 \mathrm{mM}$ imidazole for $1 \mathrm{~min}$. The grids were then washed $6 \mathrm{X}$ with either water or uranyl acetate and dried. $100 \mu \mathrm{M}$ GTPyS (Sigma) was pre-incubated at room temperature for 30 minutes with complex before application to the grid. SEC purification of the gold-labeled complexes was unsuccessful, as the label bound to retained residual protein in the column. 


\title{
Chapter 3 \\ A Membrane-Associated A2A Adenosine Receptor Dimer Revealed by \\ Two-Dimensional Electron Crystallography
}

(To be submitted as a manuscript for publication)

\begin{abstract}
Expressed highly in the brain and heart, the $\mathrm{A}_{2 \mathrm{~A}}$ adenosine receptor is a member of the GPCR superfamily, and responds to xanthine alkaloids such as caffeine in addition to adenosine. Until now, direct structural information regarding GPCRs in the native environment of the lipid membrane has been limited to rhodopsin. Here we present the two-dimensional crystallization of the $\mathrm{A}_{2 \mathrm{~A}}$ adenosine receptor in a lipid bilayer. A fusion protein comprised of $\mathrm{A}_{2 \mathrm{~A}} \mathrm{AR}$ with $\mathrm{T} 4$ lysozyme inserted in place of the third intracellular loop was overexpressed in $S$. frugiperda by baculovirus expression, solubilized in dodecylmaltoside and cholesterol hemisuccinate, and purified by cobalt affinity chromatography (TALON). Well-ordered two-dimensional crystals were generated after extensive dialysis in which the receptor was reconstituted into lipid bilayers. A defined combination of lipids was essential to obtaining well-diffracting single-layered crystals. The ribbon-like crystals resembled those of rhodopsin in morphology and are described by space group P222 1 with an orthorhombic unit cell of dimensions $a=75 \AA b=91 \AA, \gamma=$ $90^{\circ}$, in. Projection maps at $17 \AA$ resolution reveal a dimer of dimers, with a physiological dimer, and one anti-parallel dimer. Comparison of the molecular boundaries of the physiologic dimer with dimer packing observed in X-ray structures of GPCRs suggests that the dimer interface is comprised of interactions between helices I and VIII.
\end{abstract}




\section{Introduction}

The adenosine receptor belongs to a large superfamily of receptors, G-protein coupled receptors (GPCRs) which are responsible for conveying signals from numerous and diverse external stimuli, including peptides, hormones and small molecules, through their seven transmembrane helices to the membrane-associated heterotrimeric G-proteins [178]. The G-proteins, in turn, modulate a number of downstream intracellular effectors, controlling signaling for a plethora of important biological responses.

GPCRs are divided into four classes by sequence homology. The adenosine receptor belongs to class A, the largest and most widely studied. Four adenosine receptors are expressed in humans, $\mathrm{A}_{1}, \mathrm{~A}_{2 \mathrm{~A}}, \mathrm{~A}_{2 \mathrm{~B}}$, and $\mathrm{A}_{3}$, all of which modulate important physiological responses via G-protein coupling [179]. Within the human adenosine receptors, there is a high level of homology: 59\% between $\mathrm{A}_{2 \mathrm{~A}}$ and $\mathrm{A}_{2 \mathrm{~B}}$, and $49 \%$ between $\mathrm{A}_{1}$ and $\mathrm{A}_{3}$ [35]. Expression levels are variable and are based on tissue localization and extent of cellular stress. The endogenous agonist, adenosine, becomes available as a result of cell damage, or is generated via nucleotidase-mediated hydrolysis of extracellular ATP. Adenosine receptors are concentrated in the smooth muscle of the coronary arteries, where they play a role in ischemic conditioning [2], on immune cells such as monocytes where they play a role in modulating inflammation [2], and in the basal ganglia, where they act to control motor function via heterodimerization with the $\mathrm{D}_{2}$ dopamine receptor [1].

While the $\beta_{2}$ adrenergic receptor [180], rhodopsin [4] and the $\mu$-opioid receptor [181] can function as monomers, they have also been shown to exist as dimers. Table 1 
summarizes some of the evidence for GPCR dimers. High resolution x-ray crystal structures reveal both parallel and antiparallel dimers. In some instances, dimer interactions are mediated by well-ordered membrane components such as cholesterol and palmitic acid [11]. Whether GPCRs primarily function is as monomers or oligomers in vivo is a matter of debate. Contested as well is the physiologically-relevant dimerization interface. Competition experiments have implicated $\mathrm{H} 6$ of the $\beta_{2}$ adrenergic receptor [182] and $\mathrm{BLT}_{1}$ receptors [183] as a dimerization interface. H5 has been implicated in studies of the $\beta_{1}$ adrenergic receptor [184], the $\mathrm{D}_{2}$ dopamine receptor, the muscarinic $\mathrm{M}_{3}$ and serotonin receptors[185] [186] [187]. H8-H1 has also been implicated in a diverse set of receptors, including rhodopsin [188], CXCR4 [189], $\kappa$-opioid [190], and the $\beta_{1}$ adrenergic receptor [184]. Furthermore, crosslinking experiments for the $\beta_{1} \mathrm{AR}$ have demonstrated that both H8-H1 and H5 interfaces are physiologically relevant [184]. These two suggested interfaces agree with the model by Liang et al. [191] that describes the packing of rhodopsin based on the 2003 AFM results of Fotiadis et al [4]. Dimer packing in the subsequent xray crystal structure of rhodopsin crystal structure [192] only involved the H8-H1 interface. Although the receptor was largely delipidated and crystallized in the presence of detergent, the physiological relevance of this interface is supported by crosslinking experiments of native rhodopsin in disk membranes [193]. Understandably, local receptor or ligand concentrations, G-protein coupling and conformation-sensitive activation states may play roles in modulating these interfaces.

Until 2007, just one GPCR structure was available, that of rhodopsin, a rather unusual Class A GPCR family member as it is covalently bound to its ligand, 11-cis-retinal. 
Fortunately, advancements in membrane protein crystallization such as the development of lipidic cubic phase and sponge phase crystallization, and protein engineering, notably the intracellular T4L fusion by the Stevens lab, have facilitated the obtainment of additional GPCR crystal structures. Considering this expansive and medically important family of membrane proteins, the relatively scant amount of GPCR structural data is surprising, unless one considers a number of complicating factors: the relatively large size of the receptor, the presence of conformationally flexible loop regions, and the large hydrophobic surface area which is not easily accommodated by structural approaches that preclude interaction of the receptor with membrane lipids.

While the lipidic cubic phase mimics the environment of a lipid bilayer, twodimensional crystallization and electron crystallography permit the elucidation of membrane protein structures in lipid bilayers formed by lipids native to the cell membrane. While LCP can act as a membrane mimetic to a limited extent, its primary constituent, monoolein, cannot reproduce the thickness and curvature of the native bilayer. Here we present the reconstitution, 2D crystallization and electron microscopic examination of the $\mathrm{A}_{2 \mathrm{~A}}$ adenosine receptor in POPE/POPC bilayers. Analysis of the projection maps suggest that dimer interaction is mediated by the H8-H1 interface observed in 3D crystal structures for other GPCRs, notably the $\beta_{2} \mathrm{AR}$ and $\kappa$-opioid receptors. The results address the question of oligomerization of the $\mathrm{A}_{2 \mathrm{~A}}$ adenosine receptor, reveal the dimerization interface of the $\mathrm{A}_{2 \mathrm{~A}}$ adenosine receptor and provide a foundation for future two-dimensional crystallographic studies of GPCRs. 


\section{Results}

A diverse set of reconstitution strategies were used to generate well-diffracting single-layered crystals. Most useful were crystallization studies of a rhodopsin mutant [194], in combination with an extensive lipid screen. Two-dimensional crystals of the adenosine receptor were obtained in a limited number of conditions. The best condition
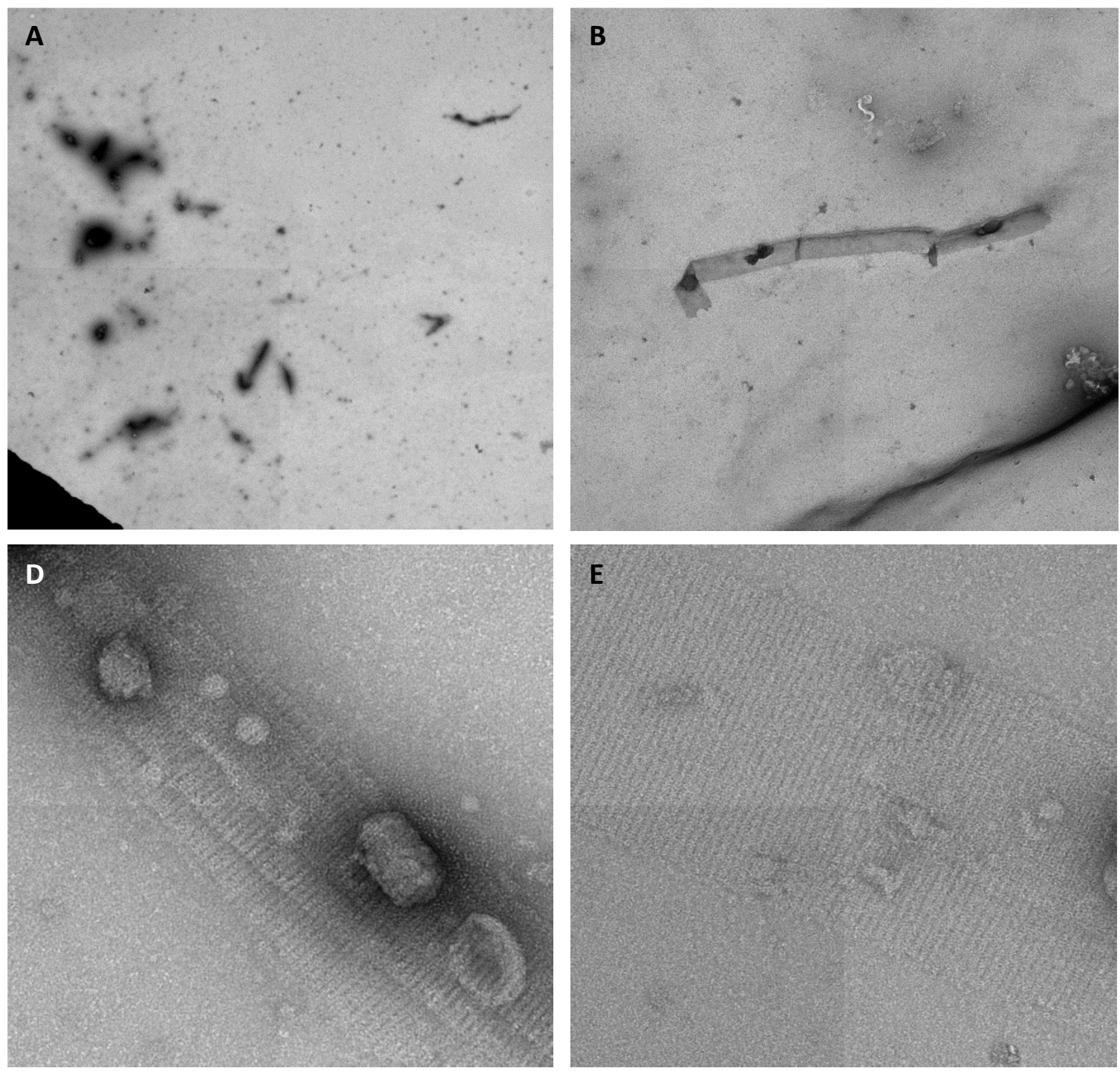

Figure 3.1. Electron micrographs of two-dimensional $A_{2 A} A R-T 4 L$ crystals. $A$, Electron micrographs showing a low magnification (4400x) field. $B$, Magnified view $(17,600 x)$ of a single ribbon-like $A_{2 A} A R-T 4 L$ crystal. $D$, Micrograph of a multilayered crystal with adherent vesicles (arrow). E, Micrograph of largely single well-ordered crystalline array. D and E, 42,000x. 
yielded ribbon-like crystals up to 2 microns long, Figure 3.1B, at a density sufficient for screening without enrichment through centrifugation, Figure 3.1A.

Cyclodextrins, Biobeads, and dialysis were employed to remove the detergent,
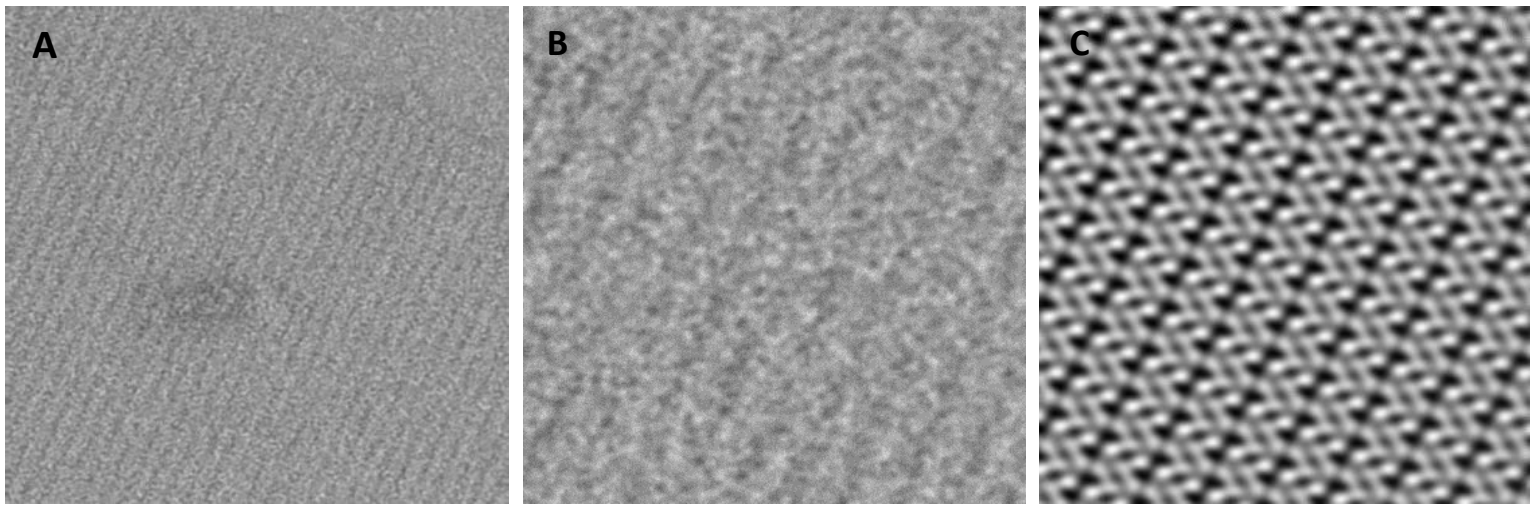

Figure 3.2. Image collection and initial processing of two-dimensional $A_{2 A} A R-T 4 L$ crystals. $A$, Electron micrograph showing regular crystalline arrays of $A_{2 A}-T 4 L$. Magnification 42,000. Enlarged $4 x$ to show detail. $B$, Area of $A$, enlarged to show detail. High signal to noise due to the low electron dose. $C$, Fourier filtered area shown in $B$.

DDM, with the highest crystalline order found in crystals grown by dialysis alone. DDM has a relatively low critical micelle concentration of $0.2 \mathrm{mM}$, thus transitional detergents [195] were examined as well as lipids, LPR, and divalent cations shown to affect membrane layering [196]. Three-dimensional rod or worm-like stacked structures formed at low $\mathrm{pH}$. A high protein concentration $(4 \mathrm{mg} / \mathrm{ml})$ was critical to forming well-diffracting crystals. Lipid choice and ratio was also crucial to forming flat single ribbons. Increasing POPE concentrations yielded highly folded structures that resembled chrysanthemum flowers as seen in Supplemental Figure 3.4E, while crystals grown in PC alone were sparse and highly stacked. Shorter dialysis times resulted in reconstituted vesicles with no detectable crystalline order and significant amounts of remaining detergent. Table 1 further summarizes the results of the reconstitution experiments. 

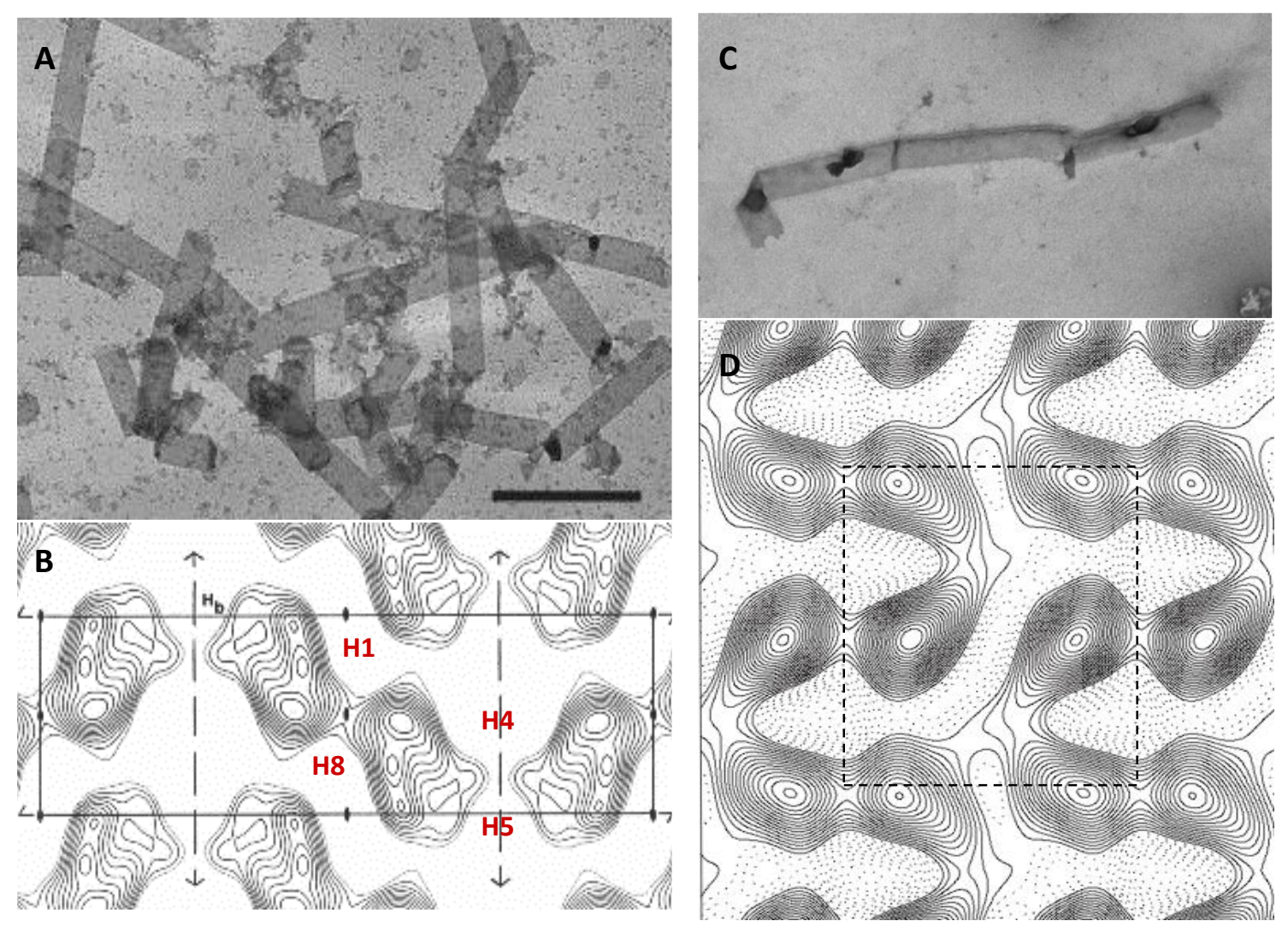

Figure 3.3. 2D crystals and projection-map comparison for rhodopsin and $A_{2 A} A R-T 4 L$ crystals. A, Electron micrograph of negatively stained rhodopsin crystals from the Journal of Molecular Biology, Vol. 285, Krebs, A., C. Villa, P.C. Edwards, and G.F. Schertler, Characterisation of an improved two-dimensional p22 $2_{1} 2_{1}$ crystal from bovine rhodopsin, copyright 1998, with permission from Elsevier. B, Rhodopsin unit cell ( $a=43 \AA, b=140 \AA$, and $y=90^{\circ}$ ) with helices numbered at dimerization interfaces for clarity from the Biophysical Journal, Vol. 68, Unger, $V$. and G. Schertler, Low resolution structure of bovine rhodopsin determined by electron cryomicroscopy, copyright 1995. C, A $2 A$ AR-T4L crystal, and D, A $2 A A R-T 4 L$ unit cell (dotted line) with dimensions: $a=75 \AA, b=91 \AA, y=90^{\circ}$. Scale bar in A is 1 micron.

The macromorphology of the two-dimensional adenosine receptor crystals (Figure 3.3C) is very similar to that of rhodopsin 2D crystals [197] (Figure 3.3A). Both unit cells contain four receptor molecules and are described by the same space group, P222 1 . The unit cells and packing of the two receptor crystals are very different however, as seen in

Figures 3.3B and 3.3D. 
Multi-layered crystals were common with both $\mathrm{A}_{2 \mathrm{~A}} \mathrm{AR}-\mathrm{T} 4 \mathrm{~L}$ (Figure 3.1C) and an N-terminal T4L- $\mathrm{A}_{2 \mathrm{~A}} \mathrm{AR}$ construct (data not shown). The stacked layers of the $\mathrm{A}_{2 \mathrm{~A}} \mathrm{AR}-\mathrm{T} 4 \mathrm{~L}$ crystals were nearly perfectly in register, and thus it was not possible to deconvolute the data during Fourier processing. Single crystals, as seen in Figure 3.1D, showed fairly isotropic scattering to approximately $17 \AA$ (Figure 3.4, left). Fourier transform of the best image of a single-layered $\mathrm{A}_{2 \mathrm{~A} A R-T 4 L}$ crystal yielded unit cell dimensions of $a=75 \AA, b$ $=91 \AA$ with $\gamma=90^{\circ}$ with four adenosine receptors per unit cell. Figure 3.4A shows a computed diffraction pattern from a single image. The Fourier filtered image seen in the right panel of Figure 3.2, reveals the composition of the strong lattice lines.
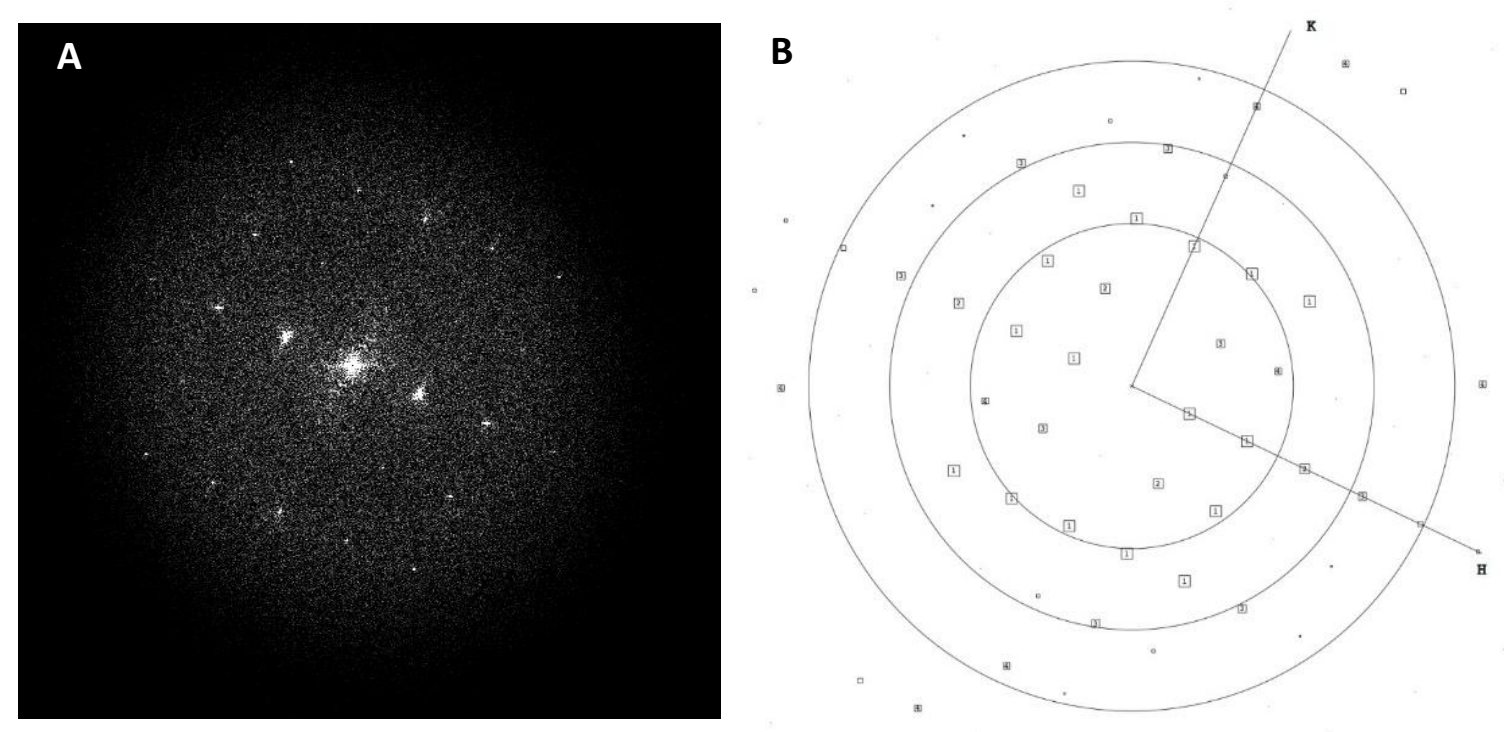

Figure 3.4: Diffraction analysis of $A_{2 A} A R-T 4 L$ crystals. A, Fourier transform of single twodimensional crystalline array showing systematic absences in odd reflections along $h$. B, Reflection intensity quality plot with resolution rings at 36,24 , and $18 \AA$. Resolution limit set at $15 \AA$. 
An intensity quotient plot is shown in Figure 3.4B, showing reflections after two rounds of unbending, defocus and CTF correction. Systematic absences of odd reflections along the $h$ axis suggest a screw axis in $b$. Examination of P1 maps show clear two fold symmetry axes along a and $b$ as well as a two-fold perpendicular to the membrane. These observations, combined with the ALLSPACE analysis results, Supplemental Figure 3.1, identify the space group as being P222 1 , a space group shared with squid and bovine rhodopsin two-dimensional crystals [198].
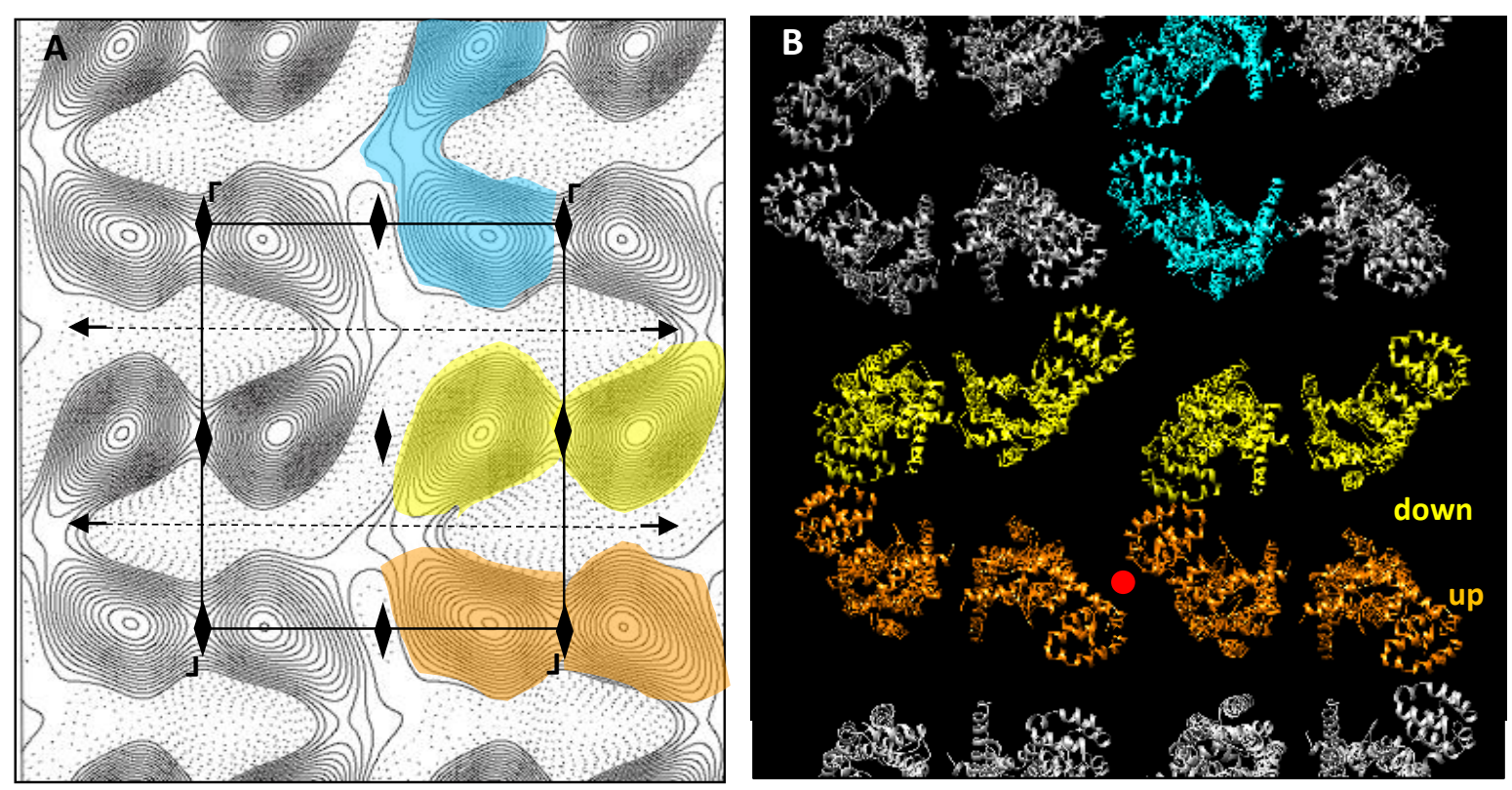

Figure 3.5. P1 projection map and proposed packing. A, the lattice lines are comprised of rows of dimers in an up/down configuration. These dimers are related by an antiparallel association (blue). Right, a packing model illustrating the rows of up/down dimers. Up dimers (orange) and down dimers (yellow). Contacts within rows alternate between receptor $\mathrm{H} 8-\mathrm{H} 1$ interfaces and $\mathrm{T} 4 \mathrm{~L}$ associations (depicted by red ball). Rows are related by antiparallel associations mediated by receptor-T4L contacts.

There are limited protein to protein contacts within the crystal. The contacts are at the parallel dimer interface and between T4L within rows, as seen in Figure 3.5. Contacts exist A P1 projection map from the best crystal, Figure 3.5, left, shows asymmetrical islands of density, in a dimeric arrangement similar to bovine rhodopsin, Figure 3.3B [199], but with an additional arc-shaped density due to T4L. The strong lateral lines in the 


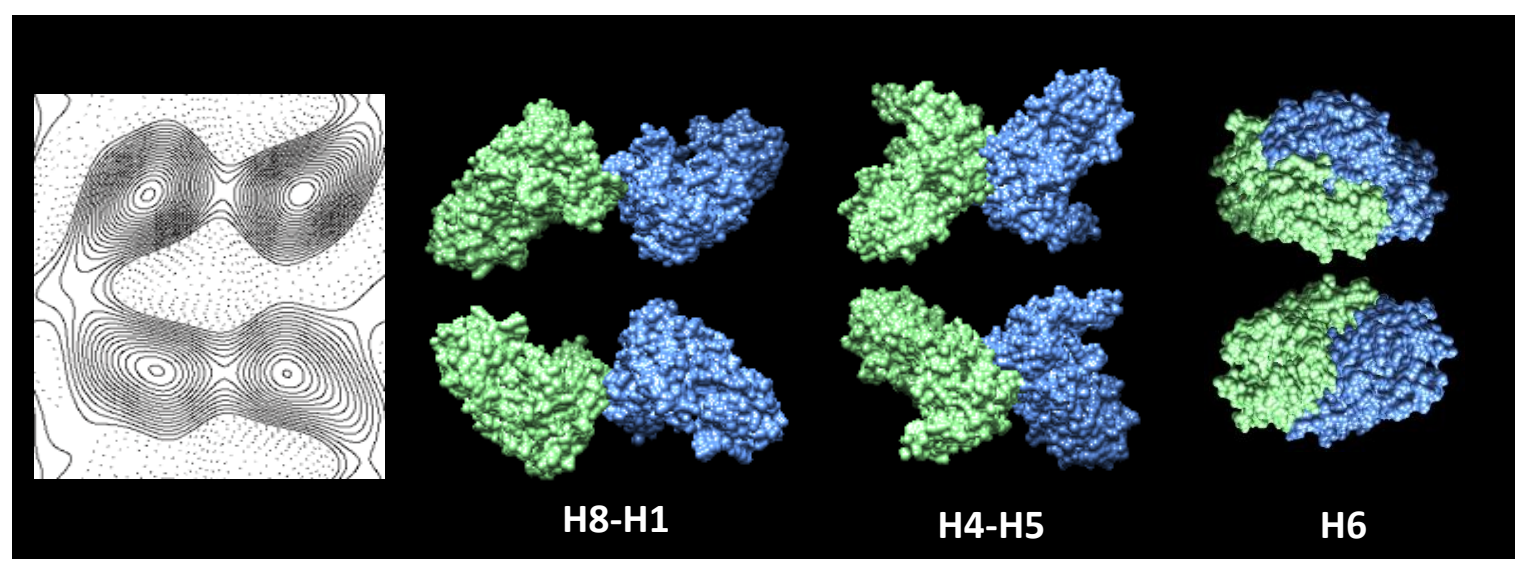

Figure 3.6. P1 projection map showing molecular boundaries of dimer and potential dimer interfaces. Surface representations of the different interfaces are as seen in 3D crystals of the $\mathrm{K}$ opioid receptor. The profile of the $\mathrm{H} 8-\mathrm{H} 1$ interface is the best fit. The amount of buried surface area would also qualitatively appear to agree with that observed in the projection map.

crystal are comprised of rows of dimers. The dimers themselves repeat in an up/down configuration along $b$, with each alternate set facing different hydrophilic sides of the membrane. Thus, there are two dimerization interfaces, a parallel dimer (yellow and orange), and an anti-parallel dimer (cyan), Figure 3.5A. The intracellular and extracellular faces of the receptor have different surface areas with the extracellular "up" face being smaller and represented by the darker density peak, and the intracellular "down" face being larger and represented by the island with the more diffuse peak of density, Figure 3.5A. Because T4L is in ICL3 and facing the respective hydrophilic sides of the crystal, T4LT4L contacts cannot be directly mediating the dimer formation. Here, dimerization may be between contacts on T4L of one receptor and extracellular contacts on its antiparallel partner. Because of the proximity of the fusion protein between $\mathrm{H} 5$ and $\mathrm{H} 6$, we cannot rule out steric hindrance or outcompetition of a native dimerization interface. 

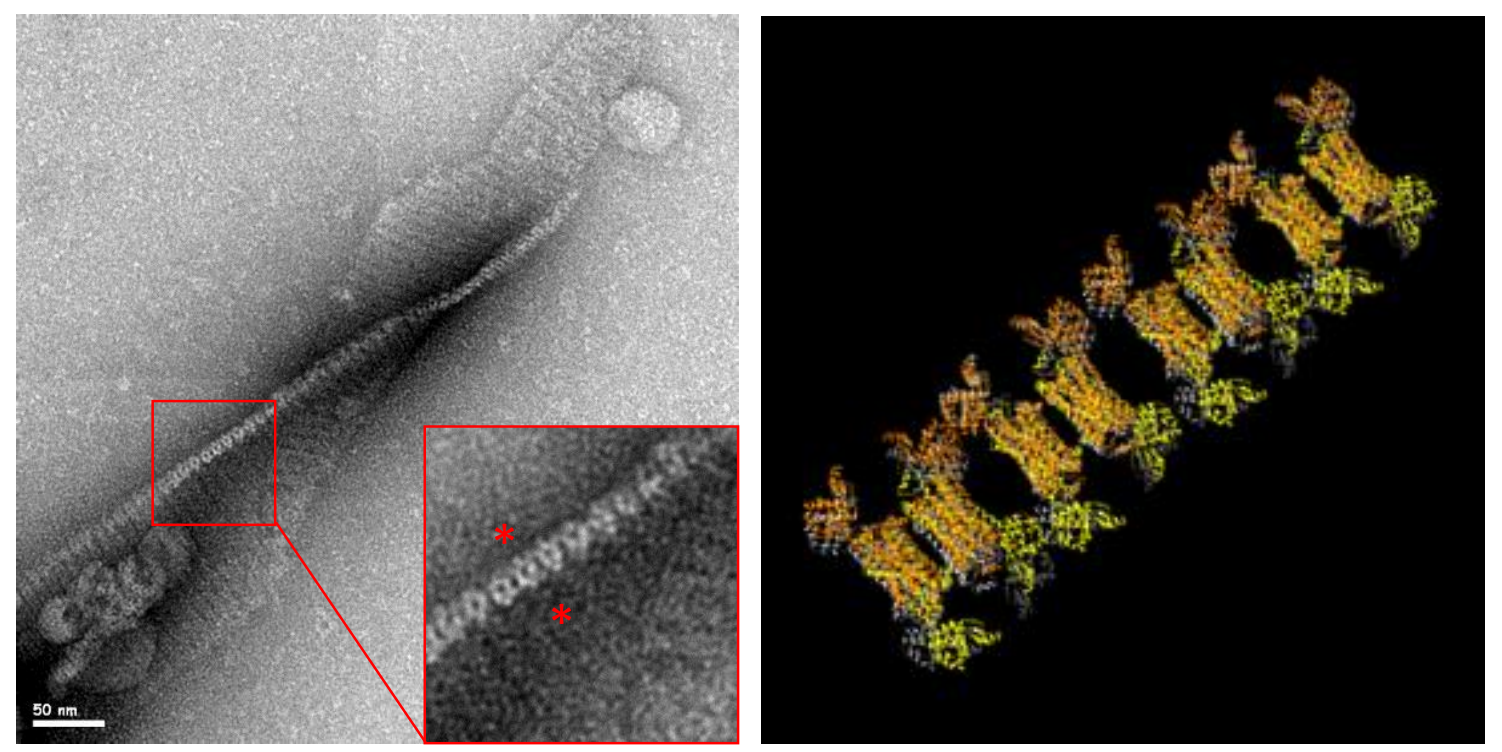

Figure 3.7. Cross-sectional views of two-dimensional $A_{2 A} A R-T 4 L$ crystals. Left, a folded single layered crystal. Measurement between protruding knobs of density*, assigned to T4L, is $117 \AA$. Expected thickness for one bilayer and two antiparallel T4L molecules oriented consistent with the 3D crystal structure of $A_{2 A} A R-T 4 L$ is $122 \AA$. Right, the packing model oriented to a side view perpendicular to the membrane based on alignment with the Fourier filtered map and the orientation of the crystal shown in the left panel. The packing arrangement as described recapitulates the areas of contrast seen in the edge view of the crystal.

The orientation of the $\mathrm{A}_{2 \mathrm{~A}} \mathrm{AR}-\mathrm{T} 4 \mathrm{~L}$ within the lipid bilayer is confirmed by rare lateral views of the crystal perpendicular to the membrane. The crystals have a propensity to curl or fold along their long edge, providing a seldom seen perspective in twodimensional crystals, Figure 3.7, left panel. Protrusions attributed to T4L can be seen on either side of the membrane (because the sample is negatively stained, the protein appears white), as seen in Figure 3.7, left panel inset. Measurements taken in ImageJ suggest that the crystal is single-layered, with a predicted measurement of $122 \AA$, and an actual measurement of $117 \AA$, for antiparallel $\mathrm{A}_{2 \mathrm{~A} A R-T 4 L}$ in a single lipid bilayer. Figure 3.7, right panel, shows an approximated edge-on view of the packing model, generated in Chimera. 


\section{Discussion}

Two-dimensional crystals of an $\mathrm{A}_{2 \mathrm{~A}} \mathrm{AR}-\mathrm{T} 4 \mathrm{~L}$ fusion have been obtained after

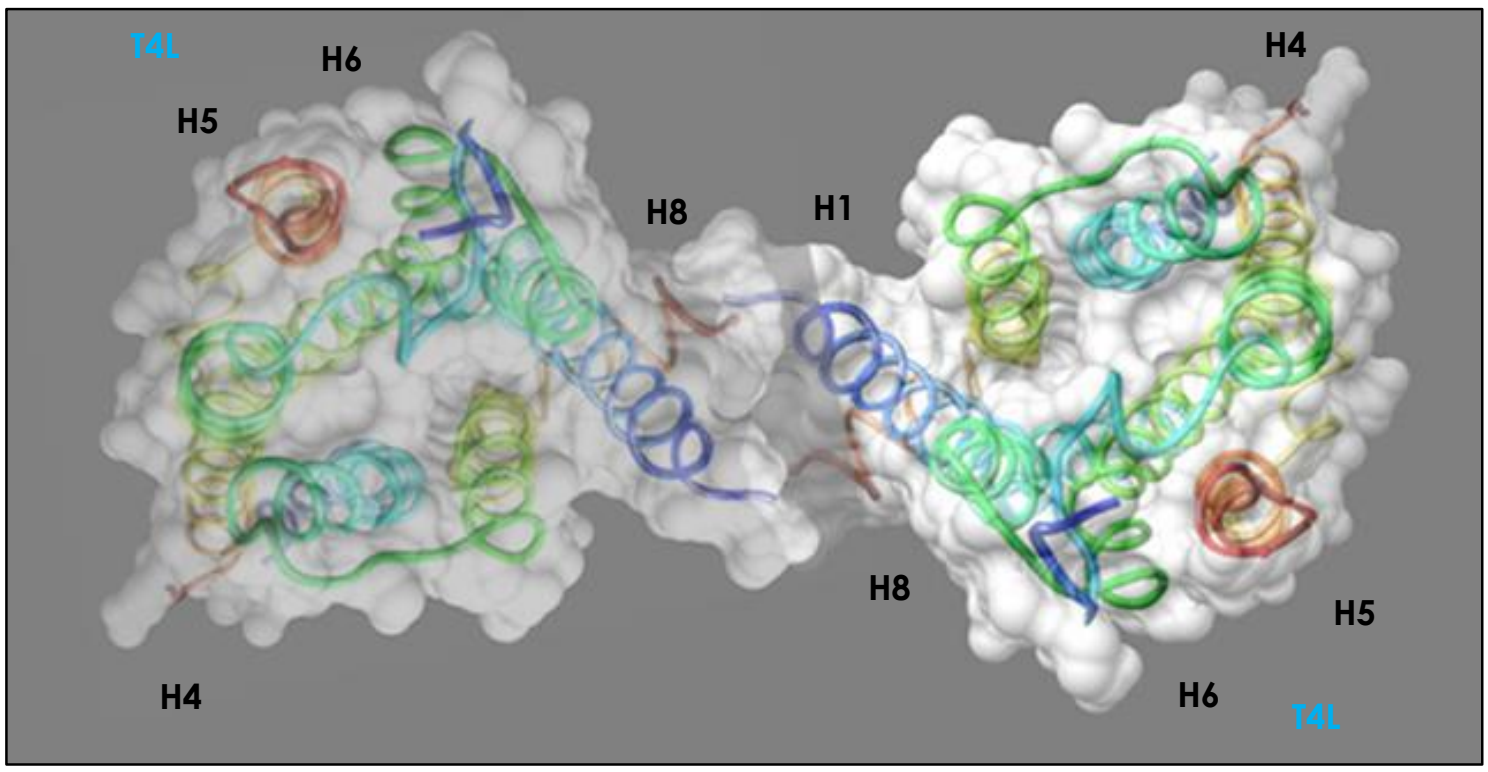

Figure 3.8. The dimer interface of the adenosine receptor in a lipid bilayer. Surface representation of the K-opioid receptor $\mathrm{H} 8-\mathrm{H} 1$ dimer (4DJH) docked with an aligned ribbon diagram of the $A_{2 A} A R$ (3EML). T4L has been removed for clarity.

expression by baculovirus, solubilization, purification, and reconstitution into lipid membranes by dialysis and detergent removal. In the most well-ordered crystals, the lipid composition is 3:7 POPE:POPC, which is similar to the proportion of PE:PC in the cell membrane, 3:6. In addition, the solubilized protein contained 10\% cholesterol hemisuccinate. Cholesterol exists at a similar concentration in the cell membrane and has been demonstrated to be at the dimerization interface of many GPCRs [11].

Limited protein contacts are involved in crystal formation. The rows of dimers exist in an alternating up-down configuration with T4L-T4L contacts mediating contact between 
sets of dimers. By identifying the location of the T4 lysozyme, taking into account the measurements of the lateral views of the crystal, and comparing the molecular boundaries observed in the P1 density map, we can assign the interface between the anti-parallel dimers to H6. There is no T4L-T4L contact between the anti-parallel dimers as the T4L tags are located on opposite sides of the membrane, however we cannot rule out T4Lreceptor contacts at $\mathrm{H} 6$. $\mathrm{H} 6$ has been previously identified as a potential dimerization interface for the CXCR4, $\mu$-opioid, $\beta_{2} \mathrm{AR}$, and $\mathrm{BLT}_{1}$ receptors, as reported elsewhere [200]. It is possible that high receptor concentration beyond physiological levels in reconstituted detergent-solubilized receptors may drive artificial antiparallel dimerization at this interface.

An additional dimerization interface observed in the crystals exists within the tight rows of dimers along $a$ that comprise the high-contrast lateral lattice lines of the crystals. Based on the boundaries of the density, we can assign this interface to $\mathrm{H} 8-\mathrm{H} 1$, as seen in Figure 3.6 and Figure 3.8. This interface, first identified by Schertler in 1993, has been the one most frequently seen amongst parallel dimers of 3D-crystallized detergentsolubilized GPCRs. The existence of this dimerization interface in both detergentsolubilized $3 \mathrm{D}$ crystals as well as the $2 \mathrm{D}$ crystals of rhodopsin, and now the $\mathrm{A}_{2 \mathrm{~A}}$ adenosine receptor in the lipid bilayer suggests along with the biochemical literature that the H8-H1 interface may be physiologically relevant for many GPCRs. Interestingly, in self-assembly coarse-grained molecular dynamics simulations of model membranes, rhodopsin showed a preference for the $\mathrm{H} 8-\mathrm{H} 1$ interface over less stable $\mathrm{H} 4$ and $\mathrm{H} 6$ interfaces, despite it having a smaller buried surface area [158]. 
Membrane packing with a receptor dimer at the H8-H1 interface would allow for two G-proteins to bind, with only very minor steric clashes at the G $\beta \gamma$ N-terminal coiled coil region, as seen in Figure 3.9, IC view. This configuration, movement of the GaH domain would disrupt putative dimerization at the H4-H5 interface, offering an intriguing model for oligomer-modulated signaling.
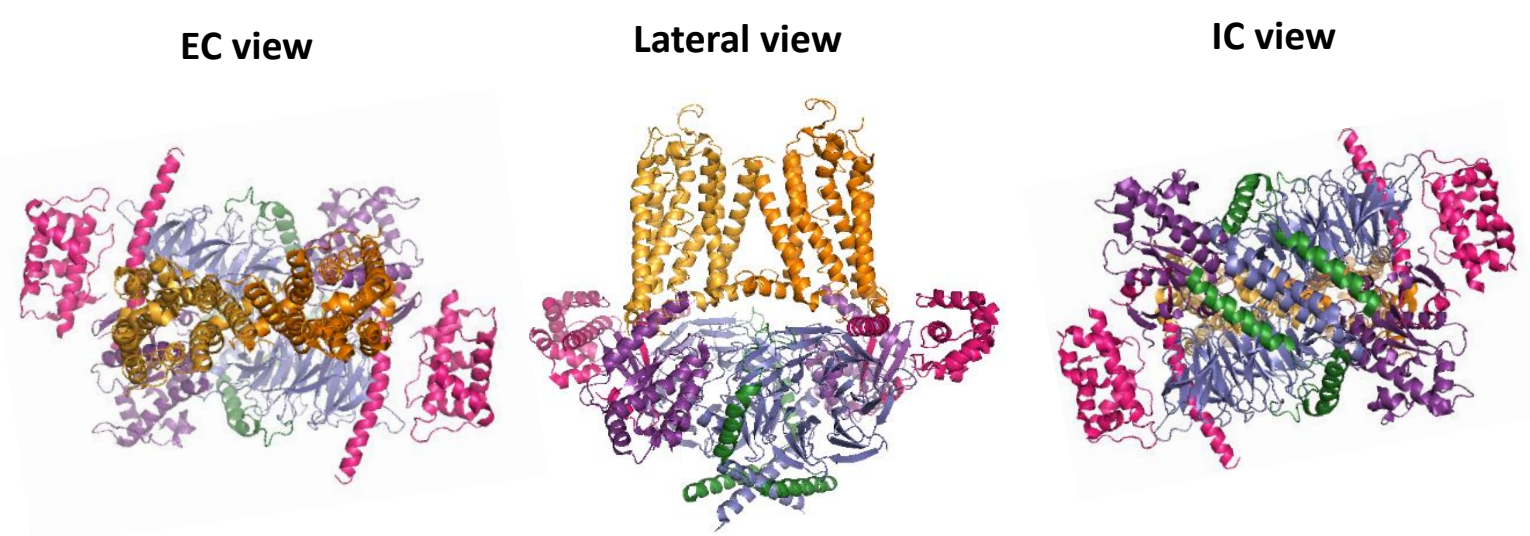

Figure 3.9. Model of an $A_{2 A} A R-A R-G s ~ H 8-H 1$ dimer. Model was generated by alignment of the $A_{2 A} A R$ (3EML) with $\beta_{2} A R$ from the $\beta_{2} A R-G s$ crystal structure (3SN6) and subsequent alignment to the H8-H1 k-opioid (4DJH) dimer. EC view, extracellular view. IC view, intracellular view. Receptor (yellow and orange), $\mathrm{G} \alpha_{\mathrm{GTPase}}$ (purple), $\mathrm{G} \alpha_{H}$ (fuchsia), $\mathrm{G} \beta$ (blue), and $\mathrm{G} \gamma$ (green).

As the $\beta_{2} \mathrm{AR}-\mathrm{Gs}$ structure is in the nucleotide-free state, it is almost certain that the overall architecture reflects a transient intermediate state in G-protein activation rather than the resting state of the receptor G-protein complex. Dimerization of GPCRs may be a transient state, acting as a stoichiometric controller and potential modulator for signaling. On comparison of $\mathrm{H} 8-\mathrm{H} 1$ interfaces of the $\mathrm{A}_{2 \mathrm{~A}} \mathrm{AR}$, the $\beta_{2} \mathrm{AR}$, and the k-opioid receptor, there is a slight but potentially significant difference. The $\mathrm{H} 8$ amphipathic helices for the $\mathrm{A}_{2 \mathrm{~A}} \mathrm{AR}$ and the $\kappa$-opioid receptor come together in a left-handed 'handshake' based on 
alignment, whereas the $\beta_{2} \mathrm{AR}$ helices come together in a right-handed 'handshake', as seen in Figure 3.10. This observation suggests a mechanism for modulation of heterodimer formation and a model wherein the presence and disposition of the palmitoylated tail in some GPCRs may play a role in combinatorial G-protein selectivity. GPCR-G-protein complex structures revealing other stages of activation will be essential in elucidating the functional effects of GPCR dimerization.
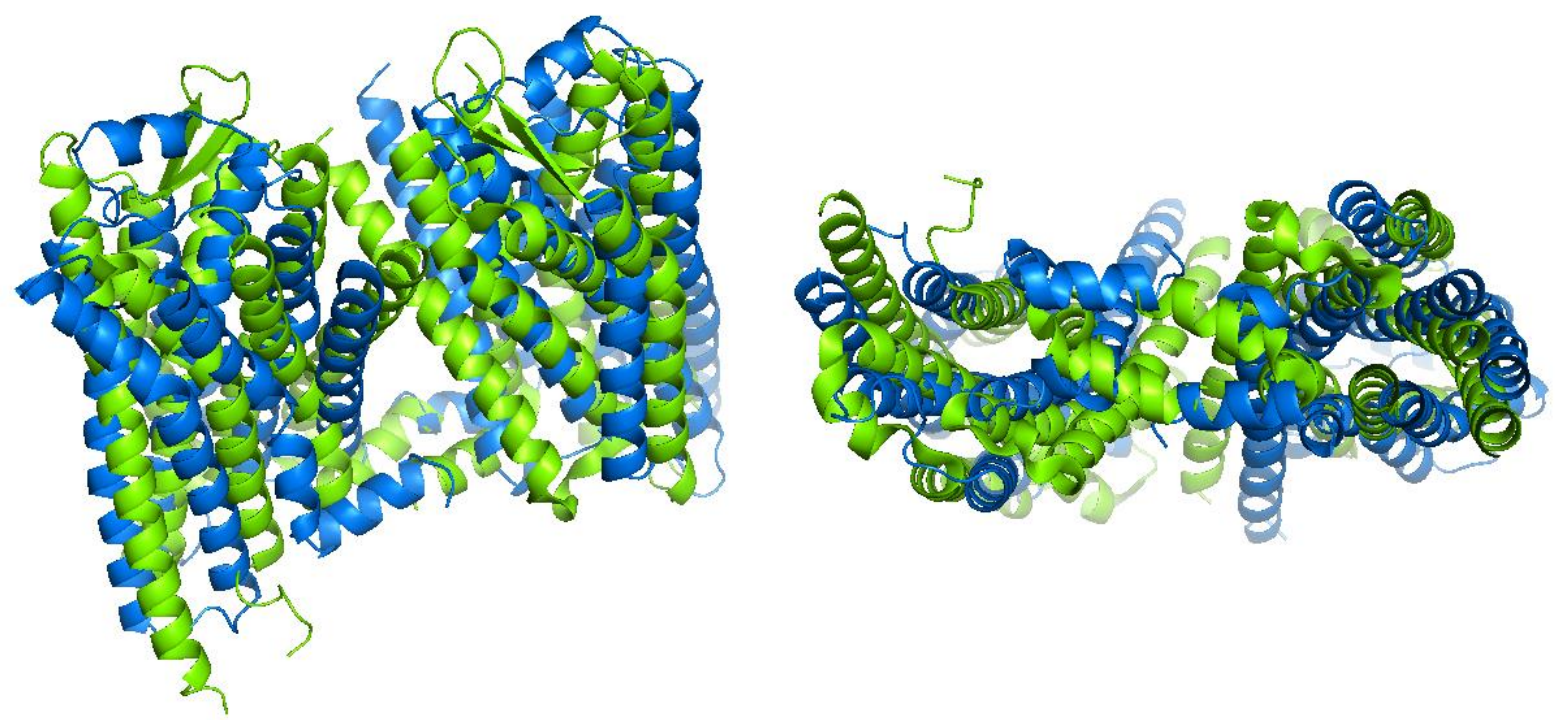

Figure 3.10. Two different H8-H1 interfaces exist in GPCRs. B2AR, blue, (2RH1) forms a righthanded $\mathrm{H} 8$ dimer, and the $\mathrm{K}$-opioid receptor, green, (4DJH) forms a left-handed $\mathrm{H} 8$ dimer. Based on alignment, the $A_{2 A} A R$ appears to form a left-handed $H 8$ dimer. Handedness relates to the angle of the $\mathrm{H} 1$ interface.

In summary, this work, based on 2D crystal data, provides a reasonable model for the dimerization of the $\mathrm{A}_{2 \mathrm{~A}}$ adenosine receptor within the lipid membrane and provides insight into the dynamic process of G-protein signaling. It is the first instance of direct structural evidence for a GPCR dimer with a non-diffusible ligand within the context of a 
lipid membrane and lays the foundation for the obtainment of $3 \mathrm{D}$ projection maps of the adenosine receptor in the native milieu of a lipid bilayer.

\section{Materials and Methods}

\section{Expression and purification}

Recombinant Baculovirus, autographa californica nuclear polyhedrosis virus (AcNPV) containing $\mathrm{A}_{2 \mathrm{~A}} \mathrm{AR}$ with a T4 lysozyme fusion (gift of Dr. Ray Stevens, The Scripps Research Institute), was used to infect clonal Spodoptera frugiperda cells. Cell pellets were homogenized by Dounce homogenization on ice using 40 strokes and washed four times, twice with a low salt $(20 \mathrm{mM} \mathrm{NaCl})$ buffer and twice with a buffer containing high salt $(800 \mathrm{mM} \mathrm{NaCl})$ and solubilized for 2 hours with $0.5 \%$ DDM (Anatrace) and $0.1 \%$ cholesterol hemiscuccinate (SIGMA) overnight at $4^{\circ} \mathrm{C}$. Pellets were obtained by centrifugation with a Ti45 rotor at 36,000 rpm for 30 minutes in a Beckman ultracentrifuge (model Optima LE-80K). Following centrifugation, the lysate was incubated with $2 \mathrm{ml}$ of resuspended TALON beads (Clontech) at $4^{\circ} \mathrm{C}$ overnight with rocking. Beads were washed twice with $10 \mathrm{ml}$ of a buffer comprised of $50 \mathrm{mM}$ HEPES pH 7.5, $800 \mathrm{mM} \mathrm{NaCl}, 10 \%$ glycerol, $10 \mathrm{mM} \mathrm{MgCl}_{2}, 25 \mathrm{mM}$ imidazole, $4 \mathrm{mM}$ theophylline and $0.1 \% \mathrm{DDM} / 0.02 \%$ CHS with $8 \mathrm{mM}$ ATP (Wash 1) or $0.05 \%$ DDM/0.001\% CHS (Wash 2). Solubilized receptor constructs were eluted with a final concentration of $220 \mathrm{mM}$ imidazole, $25 \mathrm{mM}$ $\mathrm{A}_{2 \mathrm{AAR}}$ agonist UK-432097 (a gift from Ray Stevens) and $0.025 \% \mathrm{DDM}$ and $0.005 \% \mathrm{CHS}$ in the wash buffer described above. Receptors were concentrated in a Vivaspin centrifugal concentration device (GE Healthcare) with a molecular weight cutoff at $30,000 \mathrm{kD}$ at $4^{\circ} \mathrm{C}$. 


\section{Two-dimensional crystallization trials}

$\mathrm{A}_{2 \mathrm{~A}} \mathrm{AR}-\mathrm{T} 4 \mathrm{~L}$ at $1-4 \mathrm{mg} / \mathrm{ml}$ was mixed at a 1:1 volume ratio with $1 \mathrm{mg} / \mathrm{ml}$ lipid or lipid mixture pre-solubilized by addition of DDM/CHS and incubated at room temperature for one hour. A stock 10\% DDM/2\% CHS solution was used to 'wet' lipids in amber glass vials that had been dried under argon, following which buffer comprised of $20 \mathrm{mM}$ HEPES $\mathrm{pH}$ 7.5, $100 \mathrm{mM}$ sodium chloride was added. The most successful trial utilized a lipid mixture of 3 parts 1-palmitoyl-2-oleoyl-sn-glycero-3-phosphoethanolamine (Avanti) to 7 parts 1-palmitoyl-2-oleoyl-sn-glycero-3-phosphocholine (Avanti), 3:7 POPE:POPC. Following incubation, $20 \mu \mathrm{l}$ of the lipid-receptor mixture was added to clean dialysis buttons (Hampton Research) and dialysis membrane (Spectrum) with a 10,000 molecular weight cutoff was applied to button manually with an o-ring. Buttons were placed in 100 $\mathrm{ml}$ crystallization buffer (20 mM HEPES pH 7.5, $100 \mathrm{mM}$ potassium chloride, $10 \mathrm{mM}$ magnesium chloride, $1 \mathrm{mM}$ DTT and $3 \mathrm{mM}$ sodium azide) in flat-bottomed jars with stir bar. Daily buffer exchanges were made for 18 days with stirring at room temperature. On the last day of dialysis, the button in dialysis buffer was brought to $37^{\circ} \mathrm{C}$ over the course of 1 hour and allowed to cool to room temperature. Solution was removed and placed in $0.5 \mathrm{ml}$ Eppendorf tubes and applied to grids (next section) or stored at $4^{\circ} \mathrm{C}$. Initial highly layered sheet-like crystals of T4L-A ${ }_{2 A} A R$ were grown with egg PC (Avanti) at $18^{\circ} \mathrm{C}$ using an L:P of 1:1, with a receptor concentration of $1 \mathrm{mg} / \mathrm{ml}$ in a crystallization buffer containing 20 mM HEPES pH 7.3, $100 \mathrm{mM}$ sodium chloride, $10 \mathrm{mM}$ magnesium chloride, $4 \mathrm{mM} \beta$ mercaptoethanol, $4 \mathrm{mM}$ DTT, $2.5 \%$ isopropanol and $3 \mathrm{mM}$ sodium azide, with daily 100 ml buffer exchanges for 28 days. Supplemental Table 3.1 describes some of the diverse parameters sampled in determination of crystallization conditions. 


\section{Electron microscopy}

A $3 \mu \mathrm{l}$ aliquot of the membrane suspension was applied to glow-discharged, carbon and colloidion coated 300 mesh copper grids (Electron Microscopy Services) for 2 minutes, blotted with Whatman grade 2 paper, and negatively stained with two drops of $2 \%$ depleted uranyl acetate (Ted Pella), blotted and dried. Grids were screened at room temperature with an accelerating voltage of $120 \mathrm{kV}$ on an FEI F20 or FEI Tecnai G2 and images were recorded with GATAN 4k x 4k or 2k x 2k Ultrascan CCD cameras respectively. Ribbonlike crystals were visible at 11,000x magnification in low dose and lattice lines were observed at 26,000x magnification. The adherence of small membrane vesicles aided the identification of crystals.

\section{Image processing}

Out of 113 images, 13 displayed discrete reflections that warranted further analysis. 3 of these images contained single-layered crystals. Images were processed using MRC software as implemented in 2DX [201]. Coherently diffracting regions of single-layered crystals were masked, CTF corrections were performed, and two rounds of lattice "unbending" were applied. The data from the two isomorphous crystals were merged with unit cell dimensions $78 \AA$ x $93 \AA, \gamma=90^{\circ}$. See ALLSPACE statistics, Supplemental Figure 3.1A and IQ plot, Supplemental Figure 3.1B, for merged crystals. Due to the complex symmetry, phase origins were set manually. 
ALLSPACE analysis results for multilayered crystals were poor as seen in Supplemental Figure 3.2. The pathological multilayering was particularly obvious during image processing. Because the layers were in near perfect register, filtered images had a blurry appearance as seen in Supplemental Figure 3.3D. Multilayering was also evident in the Fourier filtered projection maps, as shown in Supplemental Figure 3.3F, in which the receptor dimers (Supplemental Figure 3.3E) were no longer clearly demarcated and the lattice took on a rope-like appearance. 


\begin{tabular}{|c|c|c|c|c|c|}
\hline CONSTRUCT & \multicolumn{2}{|c|}{ DETERGENTLPR } & LIPID & ADDITIVE & RESULTS \\
\hline A2a-T4L & $\mathrm{DDM} / \mathrm{CHS}$ & 0.25 & PCHSPC & & reconstituted ribbons, weak order \\
\hline A2a-T4L & $\mathrm{DDM} / \mathrm{CHS}$ & 0.25 & 3:7 POPE:POPC & & ribbon-like xts, reconstituted vesicles, filaments \\
\hline A2a-T4L & $\mathrm{DDM} / \mathrm{CHS}$ & 1 & 3:7 POPE:POPC & & reconstituted vesicles, crystalline arrays \\
\hline A2a-T4L & $\mathrm{DDM} / \mathrm{CHS}$ & 0.25 & E. coli polar lipids & & multilayered vesicles, crystalline arrays \\
\hline A2a-T4L & $\mathrm{DDM} / \mathrm{CHS}$ & 0.5 & PCCPC & & vesicles with crystalline arrays \\
\hline A2a-T4L & $\mathrm{DDM} / \mathrm{CHS}$ & 1 & PCCPC & & thick tubes \\
\hline A2a-T4L & $\mathrm{DDM} / \mathrm{CHS}$ & 0.5 & E. coli total lipids & & vesicles with crystalline arrays \\
\hline A2a-T4L & $\mathrm{DDM} / \mathrm{CHS}$ & 1 & E. coli total lipids & & vesicles \\
\hline A2a-T4L & $\mathrm{DDM} / \mathrm{CHS}$ & 0.25 & $6: 4$ POPE:POPC & & reconstituted vesicles \\
\hline A2a-T4L & $\mathrm{DDM} / \mathrm{CHS}$ & 0.33 & 6:4 POPE:POPC & & flower-like crystals, reconstituted vesicles \\
\hline A2a-T4L & $\mathrm{DDM} / \mathrm{CHS}$ & 0.66 & 6:4 POPE:POPC & & reconstituted vesicles, aggregates \\
\hline A2a-T4L & $\mathrm{DDM} / \mathrm{CHS}$ & 1 & 6:4 POPE:POPC & & aggregates \\
\hline A2a-T4L & $\mathrm{DDM} / \mathrm{CHS}$ & 1.5 & 6:4 POPE:POPC & & aggregates \\
\hline A2a-T4L & $\mathrm{DDM} / \mathrm{CHS}$ & 2.3 & 6:4 POPE:POPC & & reconstituted vesicles \\
\hline A2a-T4L & $\mathrm{DDM} / \mathrm{CHS}$ & 4 & 6:4 POPE:POPC & & reconstituted vesicles \\
\hline A2a-T4L & $\mathrm{DDM} / \mathrm{CHS}$ & 9 & 6:4 POPE:POPC & & reconstituted vesicles \\
\hline A2a-T4L & $\mathrm{DDM} / \mathrm{CHS}$ & 0.25 & 2:8 POPE:POPC & & reconstituted vesicles \\
\hline A2a-T4L & $\mathrm{DDM} / \mathrm{CHS}$ & 0.33 & 2:8 POPE:POPC & & vesicles \\
\hline A2a-T4L & $\mathrm{DDM} / \mathrm{CHS}$ & 0.66 & 2:8 POPE:POPC & & vesicles \\
\hline A2a-T4L & $\mathrm{DDM} / \mathrm{CHS}$ & 1 & 2:8 POPE:POPC & & reconstituted vesicles \\
\hline A2a-T4L & $\mathrm{DDM} / \mathrm{CHS}$ & 1.5 & 2:8 POPE:POPC & & ribbbons, vesicles \\
\hline A2a-T4L & $\mathrm{DDM} / \mathrm{CHS}$ & 2.3 & 2:8 POPE:POPC & & aggregates \\
\hline A2a-T4L & $\mathrm{DDM} / \mathrm{CHS}$ & 4 & 2:8 POPE:POPC & & aggregates \\
\hline A2a-T4L & $\mathrm{DDM} / \mathrm{CHS}$ & 9 & 2:8 POPE:POPC & & lipid crystals \\
\hline A2a-T4L & $\mathrm{DDM} / \mathrm{CHS}$ & 0.1 & E. coli polar lipids & & worms, filaments, ordered arrays \\
\hline A2a-T4L & $\mathrm{DDM} / \mathrm{CHS}$ & 0.5 & E. coli polar lipids & & irregular reconstituted vesicles \\
\hline A2a-T4L & $\mathrm{DDM} / \mathrm{CHS}$ & 9 & E. coli polar lipids & & aggregates \\
\hline T4L-A2a & $\mathrm{DDM} / \mathrm{CHS}$ & 1 & Egg PC & MPD & aggregates \\
\hline T4L-A2a & $\mathrm{DDM} / \mathrm{CHS}$ & 1 & Egg PC & ADONIS IgM worms & \\
\hline T4L-A2a & $\mathrm{DDM} / \mathrm{CHS}$ & 1 & Egg PC & $10 \mathrm{mM} \mathrm{NiCl} 2$ islands & \\
\hline T4L-A2a & $\mathrm{DDM} / \mathrm{CHS}$ & 1 & Egg PC & $100 \mathrm{mM} \mathrm{MgCl} 2$ & multilayered reconstituted vesicles \\
\hline T4L-A2a & $\mathrm{DDM} / \mathrm{CHS}$ & 1 & Egg PC & anti-FLAG mab & sheets, islands \\
\hline T4L-A2a & $\mathrm{DDM} / \mathrm{CHS}$ & 1 & Egg PC & LDAO & small vesicles \\
\hline T4L-A2a & $\mathrm{DDM} / \mathrm{CHS}$ & 0.3 & POPC & OG & aggregates \\
\hline T4L-A2a & $\mathrm{DDM} / \mathrm{CHS}$ & 0.1 & Egg PC & OG & irregular reconstituted vesicles, islands \\
\hline T4L-A2a & $\mathrm{DDM} / \mathrm{CHS}$ & 3 & DOPC & OG & small reconstituted vesicles \\
\hline T4L-A2a & $\mathrm{DDM} / \mathrm{CHS}$ & 1 & Sphingomyelin & & worms \\
\hline T4L-A2a & $\mathrm{DDM} / \mathrm{CHS}$ & 1 & Brain PC & & small reconstituted vesicles \\
\hline T4L-A2a & $\mathrm{DDM} / \mathrm{CHS}$ & 1 & Heart Extract & worms, & $\mathrm{s}$, crystalline arrays \\
\hline T4L-A2a & $\mathrm{DDM} / \mathrm{CHS}$ & 1 & Egg PC & pH 6.2 & worms \\
\hline T4L-A2a & $\mathrm{DDM} / \mathrm{CHS}$ & 2 & Egg PC & & worms, crystalline arrays, reconstituted vesicles \\
\hline T4L-A2a & $\mathrm{DDM} / \mathrm{CHS}$ & 1 & Egg PC & $10 \mathrm{mM}$ Maltose & reconstituted vesicles, islands \\
\hline T4L-A2a & $\mathrm{DDM} / \mathrm{CHS}$ & 1 & Egg PC & Biobeads & sparsely reconstituted vesicles \\
\hline T4L-A2a & $\mathrm{DDM} / \mathrm{CHS}$ & 1 & Egg PC & & cyclodextrins small vesicles, worms \\
\hline
\end{tabular}

Supplemental Table 1. Diversity of screening conditions for crystal formation during reconstitution. Non-exhaustive list of trials. See Supplemental Figure 3.4 for representative micrographs of various morphologies. If not noted, $\mathrm{pH}$ is 7-7.5. 


\begin{tabular}{|c|c|c|c|c|c|c|c|c|c|c|}
\hline \multicolumn{11}{|l|}{ A } \\
\hline \multicolumn{2}{|c|}{ SPACEGROUP } & \multicolumn{2}{|c|}{$\begin{array}{l}\text { Phs.Res. (\#) } \\
\text { v.other spots } \\
\text { (90 random) }\end{array}$} & \multicolumn{2}{|c|}{$\begin{array}{l}\text { Phs.Res. (\#) } \\
\text { v.theoretical } \\
\text { (45 random) }\end{array}$} & $\mathrm{OX}$ & $O Y$ & $7 \mathrm{x}$ & $T Y$ & Target \\
\hline 1 & & 13.0 & 18 & 9.3 & 18 & & & & & \\
\hline & & $9.4 *$ & 9 & 4.7 & 18 & -502.7 & -490.2 & 0.00 & 0.00 & 18.5 \\
\hline & p12 b & 58.3 & 6 & 1.0 & 4 & -682.2 & -298.0 & 0.00 & 0.00 & 14.8 \\
\hline $3 a$ & p12 a & 26.4 & 6 & 33.4 & 4 & -607.7 & -399.8 & 0.00 & 0.00 & 14.8 \\
\hline $4 \mathrm{~b}$ & p121 b & $2.6 *$ & 6 & 0.8 & 4 & -682.7 & -490.0 & 0.00 & 0.00 & 14.8 \\
\hline $4 a$ & p121 a & 56.8 & 6 & 39.3 & 4 & -682.7 & -258.2 & 0.00 & 0.00 & 14.8 \\
\hline $5 \mathrm{~b}$ & c12 b & 58.3 & 6 & 1.0 & 4 & -682.2 & -298.0 & 0.00 & 0.00 & 14.8 \\
\hline $5 a$ & c12 a & 26.4 & 6 & 33.4 & 4 & -607.7 & $\begin{array}{r}-399.8 \\
-8.8\end{array}$ & 0.00 & 0.00 & 14.8 \\
\hline 6 & $\mathrm{p} 22 \overline{\mathrm{z}}^{\mathrm{d}}$ & $\begin{array}{l}20.4 \\
39.3\end{array}$ & 21 & $\begin{array}{r}3.8 \\
4.7\end{array}$ & 18 & -682.2 & $-130.0^{\circ}$ & 0.00 & 0.00 & $\begin{array}{l}15.3 \\
15.3\end{array}$ \\
\hline $7 \mathrm{~b}$ & p2221b & 66.6 & 21 & 38.8 & 18 & $\begin{array}{l}-682.2 \\
-592.7\end{array}$ & $\begin{array}{l}-130.0 \\
-259.0\end{array}$ & 0.00 & 0.00 & $\begin{array}{l}13.3 \\
15.3\end{array}$ \\
\hline $7 a$ & p2221a & $12.3=$ & 21 & $\begin{array}{r}0.8 \\
4.7\end{array}$ & 18 & -682.7 & -490.0 & 0.00 & 0.00 & 15.3 \\
\hline 8 & p22121 & 53.6 & 21 & 41.0 & 18 & -592.7 & -354.9 & 0.00 & 0.00 & 15.3 \\
\hline 9 & $\mathrm{c} 222$ & 39.3 & 21 & 4.7 & 18 & -682.2 & -130.0 & 0.00 & 0.00 & 15.3 \\
\hline 10 & $\mathrm{p}_{4}$ & 53.0 & 21 & 36.0 & 18 & -653.4 & -214.1 & 0.00 & 0.00 & 15.3 \\
\hline 11 & $\mathrm{p}_{4} 22$ & 49.9 & 46 & 36.5 & 18 & -655.3 & -219.9 & 0.00 & 0.00 & 14.1 \\
\hline 12 & $\mathrm{p} 4212$ & 48.9 & 46 & 43.1 & 18 & -592.7 & -162.6 & 0.00 & 0.00 & 14.1 \\
\hline 13 & & 14.31 & 6 & - & - & -606.5 & -295.0 & 0.00 & 0.00 & 13.0 \\
\hline 14 & p312 & 36.9 & 16 & 32.7 & 4 & -352.5 & -162.8 & 0.00 & 0.00 & 13.7 \\
\hline 15 & p321 & 42.7 & 19 & 30.9 & 10 & -516.6 & -205.0 & 0.00 & 0.00 & 14.4 \\
\hline 16 & $\mathrm{p} 6$ & 38.8 & 21 & 4.7 & 18 & -322.2 & -490.0 & 0.00 & 0.00 & 15.3 \\
\hline 17 & p622 & 52.1 & 44 & 4.7 & 18 & -322.2 & -130.0 & 0.00 & 0.00 & 14.1 \\
\hline
\end{tabular}

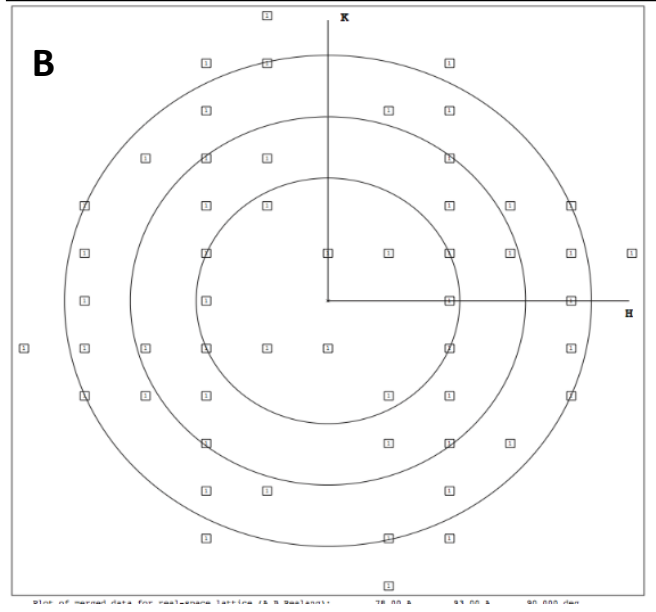

Supplemental Figure 3.1. ALLSPACE statistics and IQ plot for merged data. A, ALLSPACE statistics for merged crystals. Phase residuals indicate the plane group $\mathrm{P} 222_{1}$. B, IQ plot for two merged untilted crystals. Resolution limit set at $15 \AA$. Resolution rings at 36,24 , and $18 \AA$. Merged unit cell is $a=78 \AA \times$ $b=93$ $\AA, \gamma=90^{\circ}$. 


\begin{tabular}{|c|c|c|c|c|c|c|c|c|c|c|}
\hline \multicolumn{11}{|l|}{ A } \\
\hline \multicolumn{2}{|c|}{ SPACEGROUP } & \multicolumn{2}{|c|}{$\begin{array}{l}\text { Phs.Res. (\#) } \\
\text { v. Other spots } \\
\text { (90 random) }\end{array}$} & \multicolumn{2}{|c|}{$\begin{array}{l}\text { Phs.Res. (\#) } \\
\text { v.theoretical } \\
\text { (45 random) }\end{array}$} & $o x$ & $O Y$ & $7 \mathrm{x}$ & $T Y$ & Target \\
\hline 1 & p1 & 13.0 & 18 & 9.3 & 18 & & & & & \\
\hline 2 & $\mathrm{p}_{2}$ & $9.4 *$ & 9 & 4.7 & 18 & -502.7 & -490.2 & 0.00 & 0.00 & 18.5 \\
\hline $3 \mathrm{~b}$ & p12 b & 58.3 & 6 & 1.0 & 4 & -682.2 & -298.0 & 0.00 & 0.00 & 14.8 \\
\hline $3 \mathrm{a}$ & $\mathrm{p} 12 \mathrm{a}$ & 26.4 & 6 & 33.4 & 4 & -607.7 & -399.8 & 0.00 & 0.00 & 14.8 \\
\hline & $\mathrm{p} 12 \overline{1} \mathrm{~b}$ & $2.6=$ & 6 & 0.8 & 4 & -682.7 & -490.0 & 0.00 & 0.00 & 14.8 \\
\hline $4 a$ & $\mathrm{p} 121 \mathrm{a}$ & 56.8 & 6 & 39.3 & 4 & -682.7 & -258.2 & 0.00 & 0.00 & 14.8 \\
\hline $5 b$ & $\mathrm{c} 12 \mathrm{~b}$ & 58.3 & 6 & 1.0 & 4 & -682.2 & -298.0 & 0.00 & 0.00 & 14.8 \\
\hline $5 a$ & c12a & 26.4 & 6 & 33.4 & 4 & -607.7 & $\begin{array}{r}-399.8 \\
\end{array}$ & 0.00 & 0.00 & 14.8 \\
\hline 6 & $\mathrm{p} 22 \overline{2}$ & 39.3 & 21 & 4.7 & 18 & -682.2 & -130.0 & 0.00 & 0.00 & 15.3 \\
\hline $7 \mathrm{~b}$ & $\mathrm{p} 2221 \mathrm{~b}$ & 66.6 & 21 & 38.8 & 18 & -592.7 & -259.0 & 0.00 & 0.00 & 15.3 \\
\hline $7 a$ & p2221a & $12.3 *$ & 21 & 4.7 & 18 & -682.7 & -490.0 & 0.00 & 0.00 & 15.3 \\
\hline 8 & p22121 & 53.6 & 21 & 41.0 & 18 & -592.7 & -354.9 & 0.00 & 0.00 & 15.3 \\
\hline 9 & $\mathrm{c} 222$ & 39.3 & 21 & 4.7 & 18 & -682.2 & -130.0 & 0.00 & 0.00 & 15.3 \\
\hline 10 & $\mathrm{p}^{4}$ & 53.0 & 21 & 36.0 & 18 & -653.4 & -214.1 & 0.00 & 0.00 & 15.3 \\
\hline 11 & p422 & 49.9 & 46 & 36.5 & 18 & -655.3 & -219.9 & 0.00 & 0.00 & 14.1 \\
\hline 12 & p4212 & 48.9 & 46 & 43.1 & 18 & -592.7 & -162.6 & 0.00 & 0.00 & 14.1 \\
\hline 13 & p3 & 14.31 & 6 & -- & $=$ & -606.5 & -295.0 & 0.00 & 0.00 & 13.0 \\
\hline 14 & p312 & 36.9 & 16 & 32.7 & 4 & -352.5 & -162.8 & 0.00 & 0.00 & 13.7 \\
\hline 15 & $\mathrm{p} 321$ & 42.7 & 19 & 30.9 & $10^{\circ}$ & -516.6 & -205.0 & 0.00 & 0.00 & 14.4 \\
\hline 16 & p6 & 38.8 & 21 & 4.7 & 18 & -322.2 & -490.0 & 0.00 & 0.00 & 15.3 \\
\hline 17 & $\mathrm{p} 622$ & 52.1 & 44 & 4.7 & 18 & -322.2 & -130.0 & 0.00 & 0.00 & 14.1 \\
\hline \multicolumn{11}{|c|}{$\mathbf{B}$} \\
\hline \multirow{2}{*}{\multicolumn{2}{|c|}{ B $_{\text {SPACEGROUP }}$}} & \multirow{2}{*}{\multicolumn{2}{|c|}{$\begin{array}{l}\text { Phs.Res. (\#) } \\
\text { v.other spots } \\
(90 \text { random) }\end{array}$}} & \multirow{2}{*}{\multicolumn{2}{|c|}{$\begin{array}{l}\text { Phs.Res. (\#) } \\
\text { v.theoretical } \\
\text { (45 random) }\end{array}$}} & $\mathrm{OX}$ & $O Y$ & $\mathrm{TX}$ & $\mathrm{TY}$ & Target \\
\hline & & & & & & & & & & \\
\hline 1 & 1 & 16,6 & 18 & 119 & 18 & & & & & \\
\hline 2 & $\mathrm{p} 2$ & 12.0 * & 9 & $\begin{array}{r}1.9 \\
6.0\end{array}$ & 18 & -260.5 & 222.6 & 0.00 & 0.00 & 23.8 \\
\hline 3 & p12 b & 49.3 & 8 & 2.6 & 4 & 97.5 & 213.6 & 0.00 & 0.00 & 18.4 \\
\hline & p12a & 48.5 & 7 & 0.9 & 2 & -253.7 & -48.0 & 0.00 & 0.00 & 17.6 \\
\hline & $\mathrm{p} 12 \overline{1} \mathrm{~b}$ & 39.5 & 8 & 5.1 & 4 & -257. & 189.6 & 0.00 & 0.00 & 18.4 \\
\hline 4 & p121 a & 62.0 & 7 & 0.0 & 2 & 82.3 & 131.5 & 0.00 & 0.00 & 17.6 \\
\hline 5. & $\mathrm{c} 12 \mathrm{~b}$ & 49.3 & 8 & 2.6 & 4 & 97.5 & 213.6 & 0.00 & 0.00 & 18.4 \\
\hline 5 & c12a & 48.5 & 7 & 0.9 & 2 & -253.7 & -48.0 & 0.00 & 0.00 & 17.6 \\
\hline 6 & p $22 \overline{2}$ & 37.4 & 24 & 6.0 & 18 & -261.1 & 221.5 & 0.00 & 0.00 & 19.3 \\
\hline 7 & p2221b & 61.8 & 24 & 40.4 & 18 & -184.3 & -60.5 & 0.00 & 0.00 & 19.3 \\
\hline 7 & p2221a & 32.2 & 24 & 6.3 & 18 & -258.1 & 41.9 & 0.00 & 0.00 & 19.3 \\
\hline 8 & p22121 & 47.9 & 24 & 6.0 & 18 & -81.0 & 41.5 & 0.00 & 0.00 & 19.3 \\
\hline 9 & $\mathrm{c} 222$ & 37.4 & 24 & 6.0 & 18 & -261.1 & 221.5 & 0.00 & 0.00 & 19.3 \\
\hline 10 & $\mathrm{p}^{4}$ & 59.0 & 25 & 38.7 & 18 & -101.5 & 143.6 & 0.00 & 0.00 & 19.2 \\
\hline 11 & $\mathrm{p} 422$ & 56.6 & 54 & 39.2 & 18 & -105.3 & 3132.0 & 0.00 & 0.00 & 17.8 \\
\hline 12 & p4212 & 58.0 & 54 & 39.8 & 18 & 12.6 & $5-106.0$ & 0.00 & 0.00 & 17.8 \\
\hline 13 & p3 & $15.6 *$ & 10 & - & - & -89.6 & $5 \quad 152.4$ & 0.00 & 0.00 & 16.6 \\
\hline 14 & p312 & 53.2 & 25 & 52.3 & 6 & -103. & 152.4 & 0.00 & 0.00 & 17.4 \\
\hline 15 & p321 & 29.9 & 26 & 32.4 & 8 & -89. & 152.3 & 0.00 & 0.00 & 17.7 \\
\hline 16 & p6 & 56.2 & 29 & 38.8 & 18 & 20.7 & -97.4 & 0.00 & 0.00 & 18.8 \\
\hline 17 & p622 & 56.0 & 60 & 38.8 & 18 & 20.7 & -97.4 & 0.00 & 0.00 & 17.6 \\
\hline
\end{tabular}

Supplementary Figure 3.2. ALLSPACE analyses. ALLSPACE analysis for single-layered crystals (A) and double-layered crystals (B). A, Representative Allspace results for a single-layered crystalline array with phase residuals indicating space group P2221. B, Representative ALLSPACE results for a multi-layered crystal with poor phase residuals. 

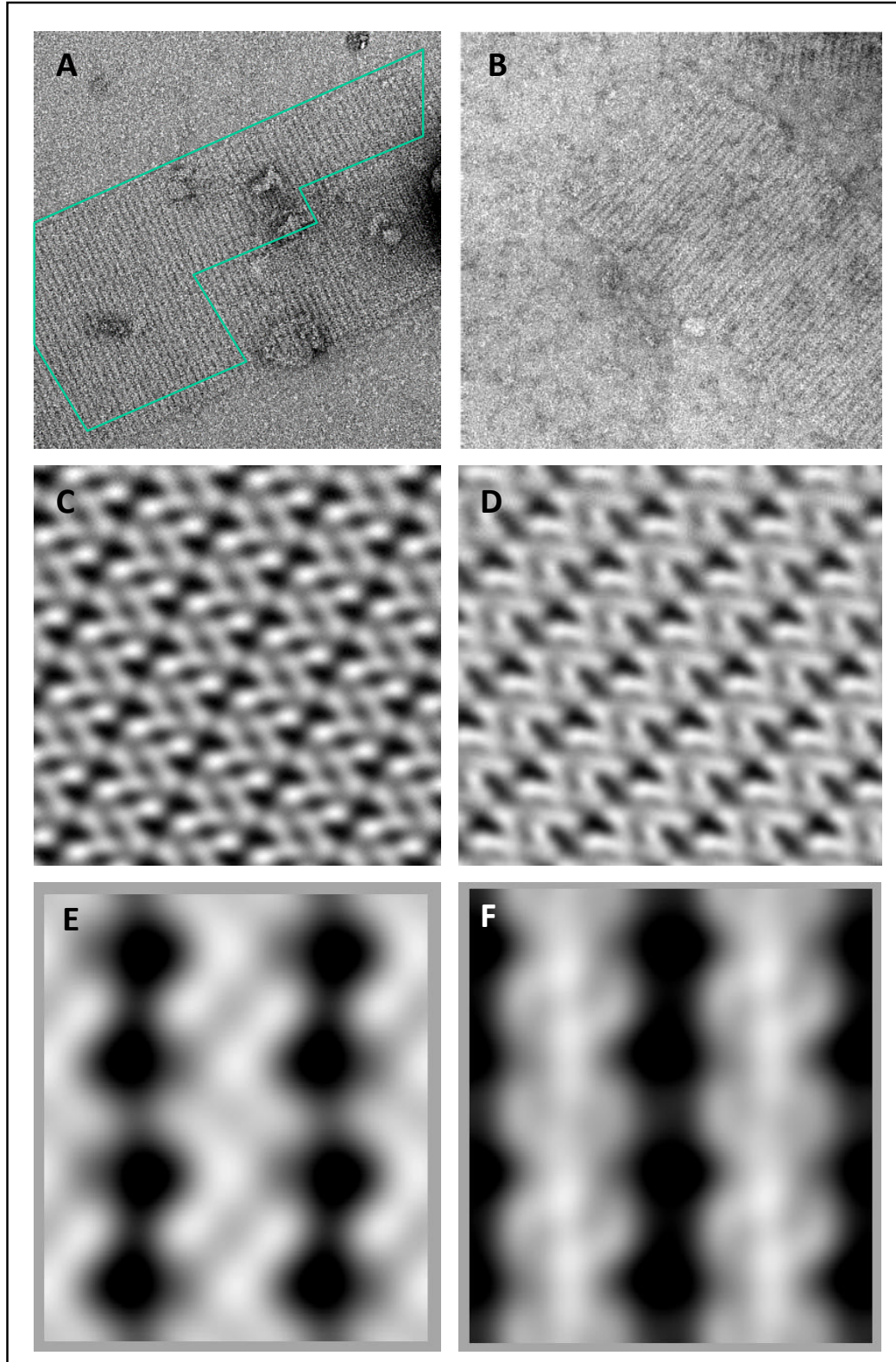

Supplemental Figure 3.3. Image processing of single and doublelayered 2D crystals of $A_{2 A} A R-T 4 L$. A, Single-layered area indicated in cyan. B, Pathological multi-layered crystal. $C$ and $D$, Fourier filtered areas from $A$ and $B$, respectively, with $D$ appearing blurry. $E$ and $F$, Fourier filtered two-dimensional $P 1$ projection maps from single-layered (E) and multi-layered crystals (F). Distinctive " $y$ "shaped T4L-mediated dimers are visible in $\mathrm{E}$, whereas in $\mathrm{H}$ they display a rope-like appearance. 

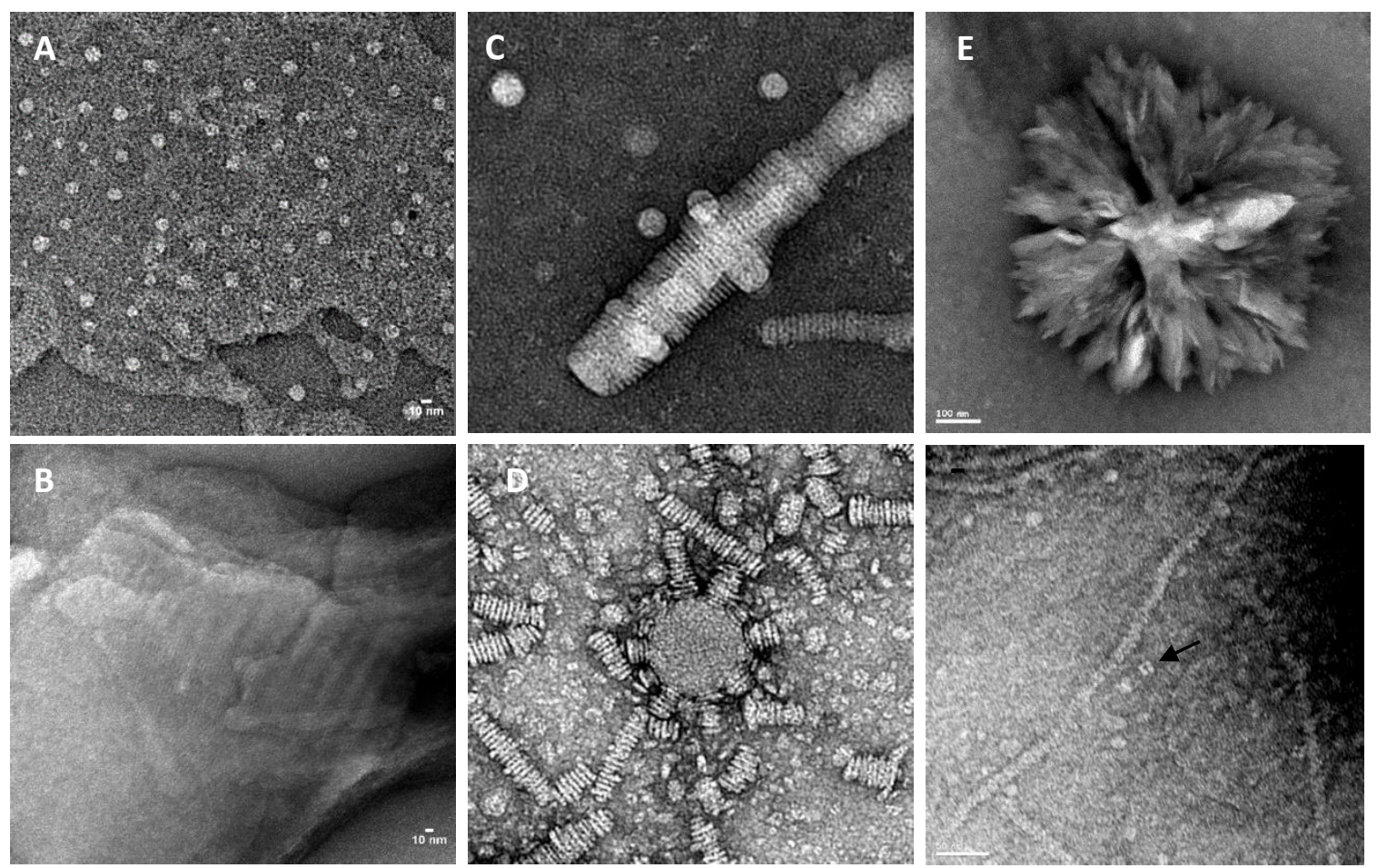

Supplemental Figure 3.4. Electron microscopic examination of adenosine receptor reconstitution. $A, T 4 L-A_{2 A} A R$ reconstituted into lipid. Receptor exists as dimers or tetramers. $B$, Highly multi-layered sheet-like crystals of T4L-A $A_{2 A} A R$ in a PC bilayer. $C$, Three-dimensional rod or worm-like structures observed in low pH (6.2) reconstitution experiments and in conditions where cyclodextrins were used to remove detergent (D). E, Chrysanthemum flower-like structures with highly folded membrane containing crystalline arrays of $A_{2 A} A R-T 4 L$, observed when a 2:8 ratio of POPE:POPC was used. $F, A_{2 A} A R-T 4 L$ filament. Arrow shows dimeric nature of subunit composition. 


\section{Chapter 4}

\section{Micro Electron Diffraction}

\section{Introduction}

Membrane protein structural biology is challenging. Expression, solubilization, purification, stabilization and crystallization are complicated by the amphipathic nature of membrane proteins: the hydrophobic membrane-embedded regions exist in a lipid bilayer environment and must be solubilized by membrane mimetics such as detergents, while the extra-membrane domains exist in an aqueous environment and are soluble in typical buffer solutions. Often, even with soluble proteins, optimization of crystallization conditions, including seeding, is confounded by disordered crystals with mosaicity. The structural biologist may be left with either showers of microcrystals, or larger mosaic crystals that fail to deliver high resolution diffraction data. The investment in high throughput equipment, detergents, and crystallization screens makes for expensive failure.

Micro electron diffraction (Micro ED) is a recently developed electron diffraction technique that utilizes the power of the electron microscope and a sensitive direct (CMOS) detector that can make use of small 3D small crystals, $0.5-1.5$ microns, which might otherwise be discarded. The technique is possible because of the extremely low dose of electrons necessary during data collection $<0.01 \mathrm{e}^{-} / \AA^{2}$ per second for thin crystals [202]. Initially using the canonical crystallization protein lysozyme, Tamir Gonen and colleagues developed the technique of micro electron diffraction, obtaining the structure of $2.9 \AA$ resolution [203] and subsequently that of catalase at $3.2 \AA$ [202]. MicroED fills a gap in 
macromolecular crystallography by enabling high-resolution structural analysis of protein crystals that are too small to analyze using conventional X-ray crystallography. The likelihood of mosaicity increases with the size of the crystal; therefore, the ability to examine micro crystals or crystal fragments reduces the chance that a mosaic area will be in the beam path.

Although Gonen et al. examined crystals with a thickness up to $3 \mu \mathrm{m}$ only crystals from $0.5-1 \mu \mathrm{m}$ yielded suitable diffraction. Crystals up to $1.5 \mu \mathrm{m}$ showed variation in diffraction quality relative to the tilt angle. Larger crystals were too thick for penetration by the electron beam. Crystals smaller than 0.5 microns were also not usable. In fact, others have attempted the concept previously using 0.1 micron crystals but were not successful in obtaining a structure [204] due to a combination of beam damage and eucentric height variation during crystal rotation or loss of symmetry due to dynamic scattering. For Gonen, ultimately three suitably sized crystal fragments were cryopreserved and frozen by plunging into liquid ethane. Multiple exposures were recorded in $1^{\circ}$ increments, tilting each crystal from $-45^{\circ}$ to $45^{\circ}$. Data from the three crystals were merged to produce the final data set.

When compared with the massive expense of synchrotron facilities and strict limitations in the availability of free electron lasers for analysis of microcrystals by X-ray diffraction, the relatively widespread availability of electron microscope facilities makes MicroED an especially appealing technique for further development. At the time of this work, the technique has been limited to crystals derived from soluble protein targets. In an attempt 
to broaden the applicability of the method to membrane proteins, I investigated the possibility of utilizing micro electron diffraction with crystals grown by all three commonly used crystallization techniques: conventional hanging drop with detergentsolubilized protein, lipidic cubic phase and crystallization in bicelles.

\section{Methods}

\section{Solubilization, purification and crystallization of A2AAR-T4L in lipidic cubic phase}

$4 \mathrm{~g}$ of prepared membranes were thawed and solubilized in $20 \mathrm{ml}$ of $50 \mathrm{mM}$ HEPES pH 7.5, $800 \mathrm{mM}$ sodium chloride, $10 \%$ glycerol, $20 \mathrm{mM}$ imidazole, $1 \mathrm{mM}$ Theophylline, with an EDTA-free protease inhibitor tablet (Roche). $2 \mathrm{mg} / \mathrm{ml}$ of iodoacetamide was added for 20 minutes and incubated on ice. DDM/CHS was added to a final concentration of $0.5 \%$ $\mathrm{DDM} / 0.1 \% \mathrm{CHS}$ and the solution was incubated with rocking at $4^{\circ} \mathrm{C}$ for 3 hours. Following centrifugation, $2 \mathrm{ml}$ of buffer-equilibrated Talon slurry in $50 \mathrm{mM}$ HEPES pH 7.5, $800 \mathrm{mM}$ $\mathrm{NaCl}, 10 \%$ glycerol, $20 \mathrm{mM}$ imidazole, $10 \mathrm{mM} \mathrm{MgCl} 2$ was added to the solubilized membranes and incubated overnight with rocking at $4^{\circ} \mathrm{C}$. Resin was added to a gravity flow column and washed with increasing imidazole concentrations, decreasing detergent concentrations and the agonist UK-432097. Purified protein was eluted using $25 \mathrm{mM}$ HEPES pH 7.5, $800 \mathrm{mM} \mathrm{NaCl}, 10 \%$ glycerol, and $220 \mathrm{mM}$ imidazole in $0.025 \%$ DDM/0.005\% CHS and 25 uM UK-432097.

LCP crystallization and optimization methods were developed by Michael Purdy of the Yeager lab. $8 \mu$ concentrated $\mathrm{A}_{2 \mathrm{~A}} \mathrm{AR}-\mathrm{T} 4 \mathrm{~L}$ was mixed with a $12 \mu \mathrm{L} 90 \%$ monoolein/10 \% 
cholesterol mixture using syringes until formation of the translucent cubic phase. For crystallization, $45 \mathrm{nl}$ of LCP was mixed with $800 \mathrm{nl}$ precipitant (31\% PEG400, 0.4 M potassium formate, $0.1 \mathrm{M}$ sodium citrate $\mathrm{pH} 4.5$ ) using an automated liquid handler. Plates were incubated at $17^{\circ} \mathrm{C}$. Crystals were harvested rapidly using a glass cutter and prepared for microscopy as described below.

Lysozyme crystals were grown as described [205].

Bicelle crystals were provided by Jake Morgan and Joshua McNamara of the laboratory of Jochen Zimmer. Bicelle preparation and crystallization performed as described elsewhere [206], with further optimization (unpublished).

\section{Cryopreservation of crystals grown by vapor diffusion and in bicelles}

Cryopreservation of crystals grown by vapor diffusion or in bicelles is relatively straightforward as the sample is not particularly viscous. Crystals are applied to carboncoated colloidion-covered copper grids by pipetting $2 \mu \mathrm{l}$ of the hanging drop or diluted sample from a sitting drop plate to the copper grid with a 1 minute incubation, quickly blotted, and flash plunging into liquid ethane with the use of a manual freezing apparatus or Vitribot (FEI). The sample is then transferred to liquid nitrogen and stored until use.

\section{Cryopreservation of crystals grown in lipidic cubic phase}

Lipidic cubic phase is extremely viscous, thus necessitating the development of crystal application methods. Multiple trials were explored in order to determine a feasible 
method for transferring the LPC-embedded crystals onto the delicate surface of the carboncoated colloidion-covered copper grids. Methods examined include direct transfer of microcrystals using conventional crystallography tools (MiTeGen), and direct application. Direct transfer often resulted in tearing of the grid surface and uneven deposition. Direct application also resulted in grid tearing from the pipette tip as well as scant deposition, as the LCP pulled away from the grid surface. Dilution of the LCP-crystals was undesirable as it converted the drop to lipidic mesophase and had the potential for disrupting the crystals. I developed a successful method of crystal transfer which utilizes a small ball of polystyrene $(\sim 3 \mathrm{~mm})$. Grasping the ball with the forceps, the ball is dabbed onto the exposed drop of LCP. The ball is then swiped in a rolling motion across the surface of a grid immobilized flat on a piece of Parafilm M (Bemis) using an additional pair of forceps. The grid is then mounted into the manual freezing device or Vitribot and flash frozen in liquid ethane, transferred to liquid nitrogen and stored. The polystyrene ball serves two purposes: it serves as a large adherent surface for the LCP sample, and it cushions the grid during sample application. As there is no fluid during application, blotting is unnecessary, however care must be taken to swipe the ball evenly across the grid surface as to avoid areas which are too thick.

\section{Electron Microscopy}

Direct micro-electron diffraction requires the use of a fast direct CMOS detector and operation of the microscope in diffraction mode. The lack of a sufficiently fast direct detector capable of imaging in diffraction mode precluded high-resolution 2D crystallography by direct methods, however we obtained preliminary results using image 
diffraction. Images were collected with an accelerating voltage of $120 \mathrm{kV}$ on the FEI Tecnai G2; at $120 \mathrm{kV}$ or $200 \mathrm{kV}$ on an FEI F20, or at $300 \mathrm{kV}$ on an FEI TITAN, with GATAN 4k x 4k or 2k x 2k Ultrascan CCD cameras under cryo-conditions.

\section{Results}

\section{Diffraction of control crystals}

Negatively-stained catalase crystals for use as an internal standard (LADD) were selected for their thinness and for their strong diffraction as a control experiment. Figure 4.1 shows a catalase crystal and its respective diffraction pattern. Approximate unit cell dimensions were not possible to calculate due to an insufficient number of spots. Lysozyme crystals were also used for control diffraction experiments. Diffraction was observed in whole crystals, although crystal fragments were used by the Gonen lab in their data collection (oral communication) due to their optimal dimensions.
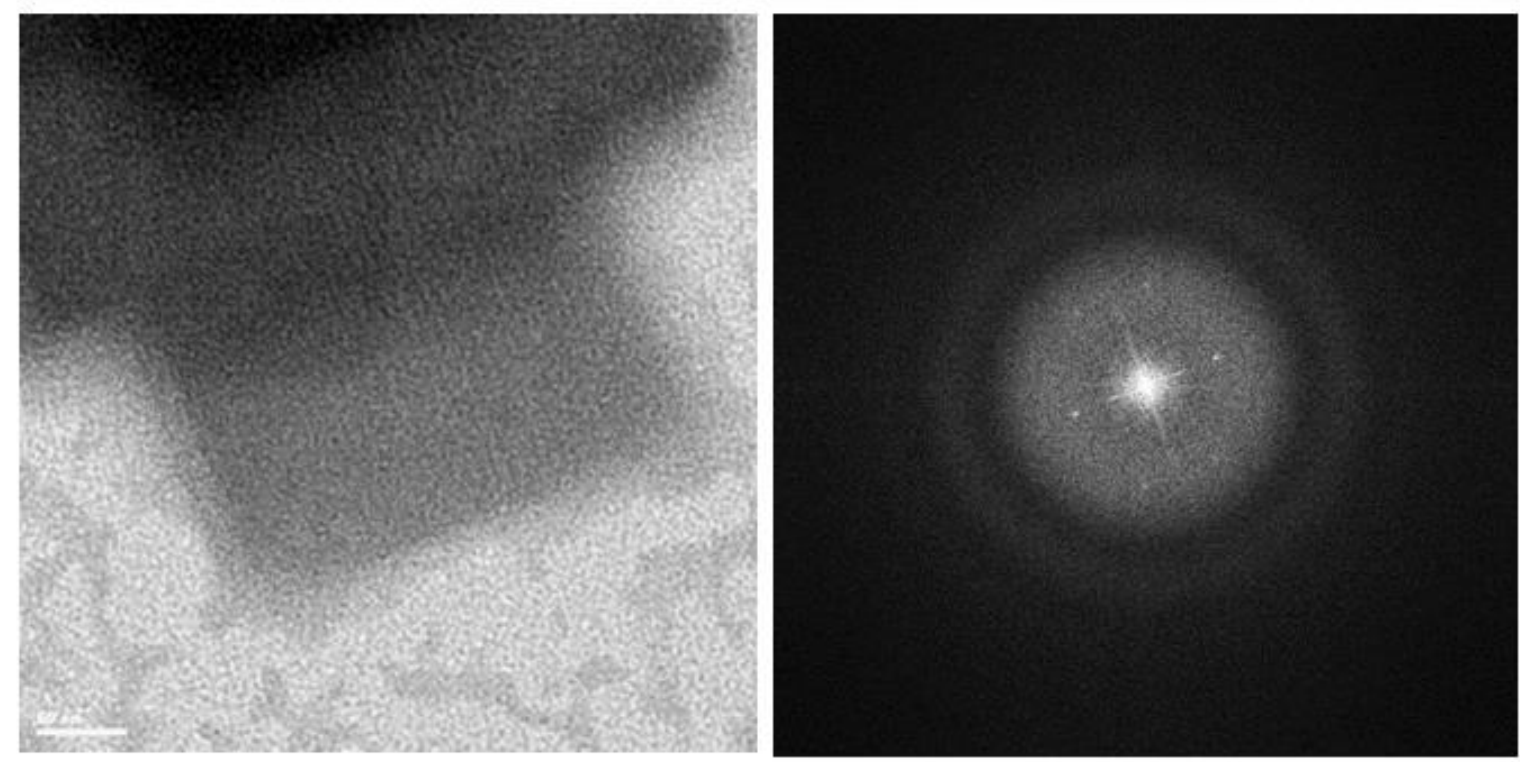

Figure 4.1. Electron micrograph and computed diffraction of 3D catalase crystals. Lattice lines in a thin 3D crystal section of twinned catalase crystals (left). Right, image diffraction of catalase crystal. 


\section{Diffraction of LCP crystals}

$\mathrm{A}_{2 \mathrm{AAR}} \mathrm{A}-\mathrm{T} 4 \mathrm{~L}$ crystals grown in LCP were examined in both negative stain as well as in vitreous ice. LCP deposition was frequently in quasi-hexagonal "islands" as seen in Figure 4.2, left. Crystals appeared dark within a bordered area comprising the LCP (Figure 2, right). Lattice lines were readily observed as seen in Figure 4.3, middle. Distorted crystals were also observed in areas where there was incomplete or no LCP embedding. No diffraction or weak diffraction spots were attained from such crystals. Figure 4.3, right, shows diffraction from a cryopreserved negatively-stained crystal of $\mathrm{A}_{2 \mathrm{~A}} \mathrm{AR}-\mathrm{T} 4 \mathrm{~L}$ grown in lipidic cubic phase. The software program 2DX was used to calculate an approximate partial unit cell, with dimensions $67 \AA$ x $71 \AA$. The unit cell dimensions for the published 3D crystals is $48 \times 79$ x $87 \AA$, by comparison. Both crystals show P2 $2_{1}$ symmetry.

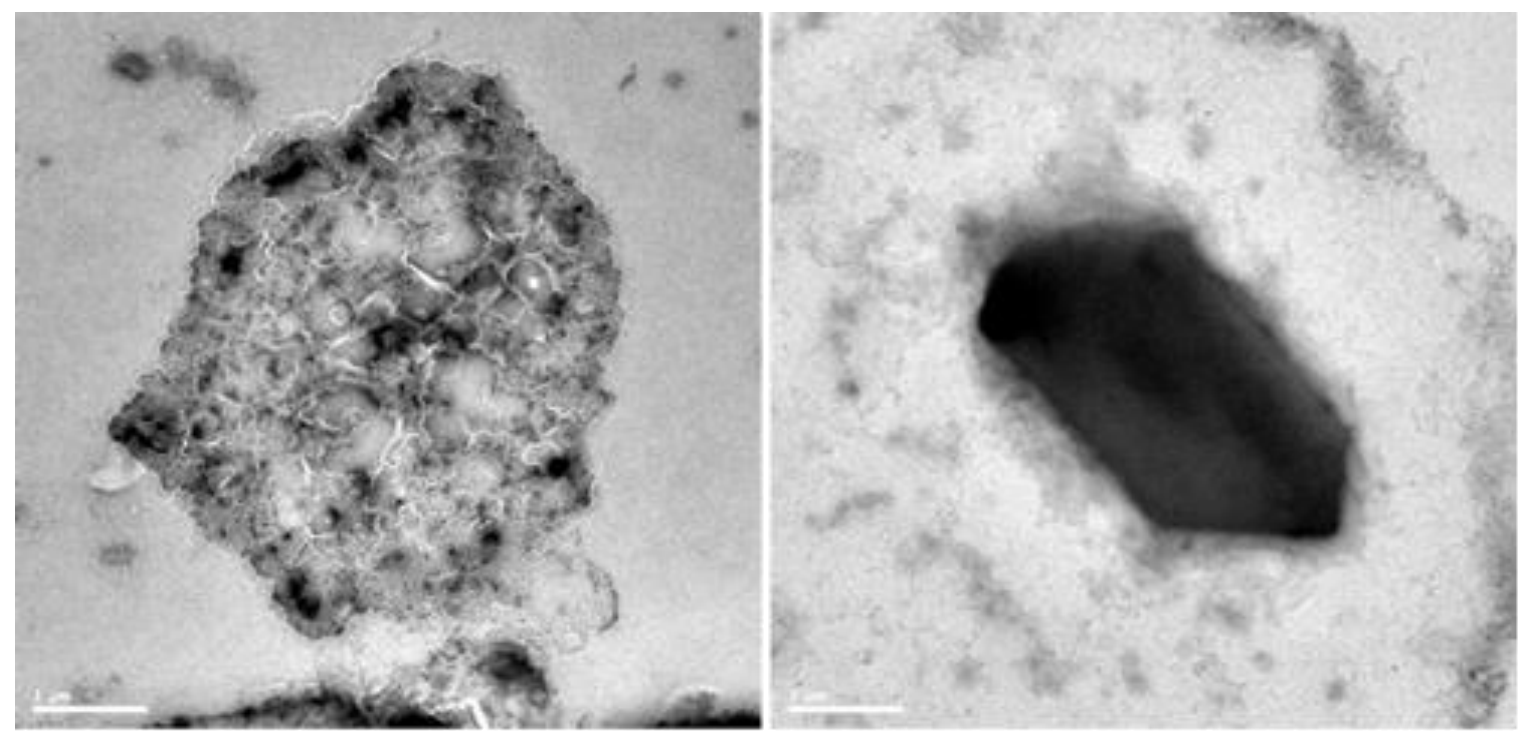

Figure 4.2. Negative stain EM of 3D A $2 A A R-T 4 L$ crystals grown in LCP. Left, LCP "island" with dark-staining $A_{2 A} A R-T 4 L$ crystals. Middle, micrograph of $A_{2 A} A R-T 4 L$ crystal at high magnification. 

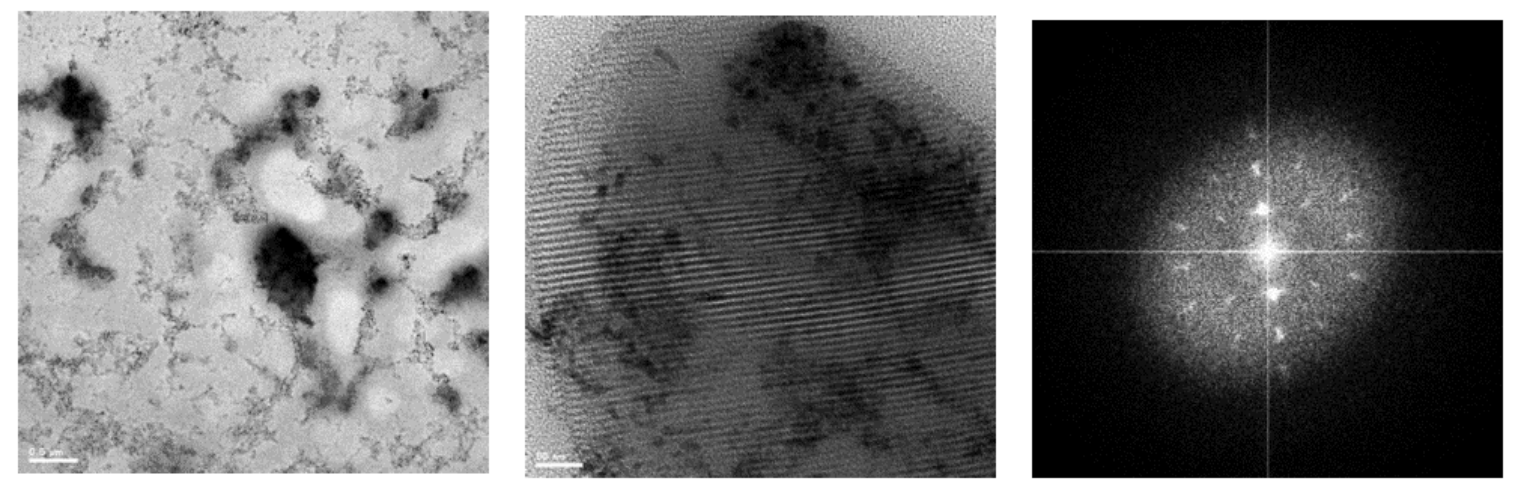

Figure 4.3. Electron microscopy and image diffraction of negatively-stained three-dimensional A $2 A_{2 A R-T 4 L}$ crystals grown in LCP. Left, micrograph of an $A_{2 A} A R-T 4 L$ crystal embedded in LCP. Middle, micrograph at higher magnification of 3D crystal showing lattice lines. Right, diffraction of crystal shown in middle image.

Figure 4.4 shows a direct diffraction image taken by Dan Shi of the Gonen lab, at Janelia Farm. The crystal (Figure 4.4, left) is incompletely embedded in the LCP. The resulting diffraction image (Figure 4.4, right) shows spots arising from buffer salts, ice, as well as protein.
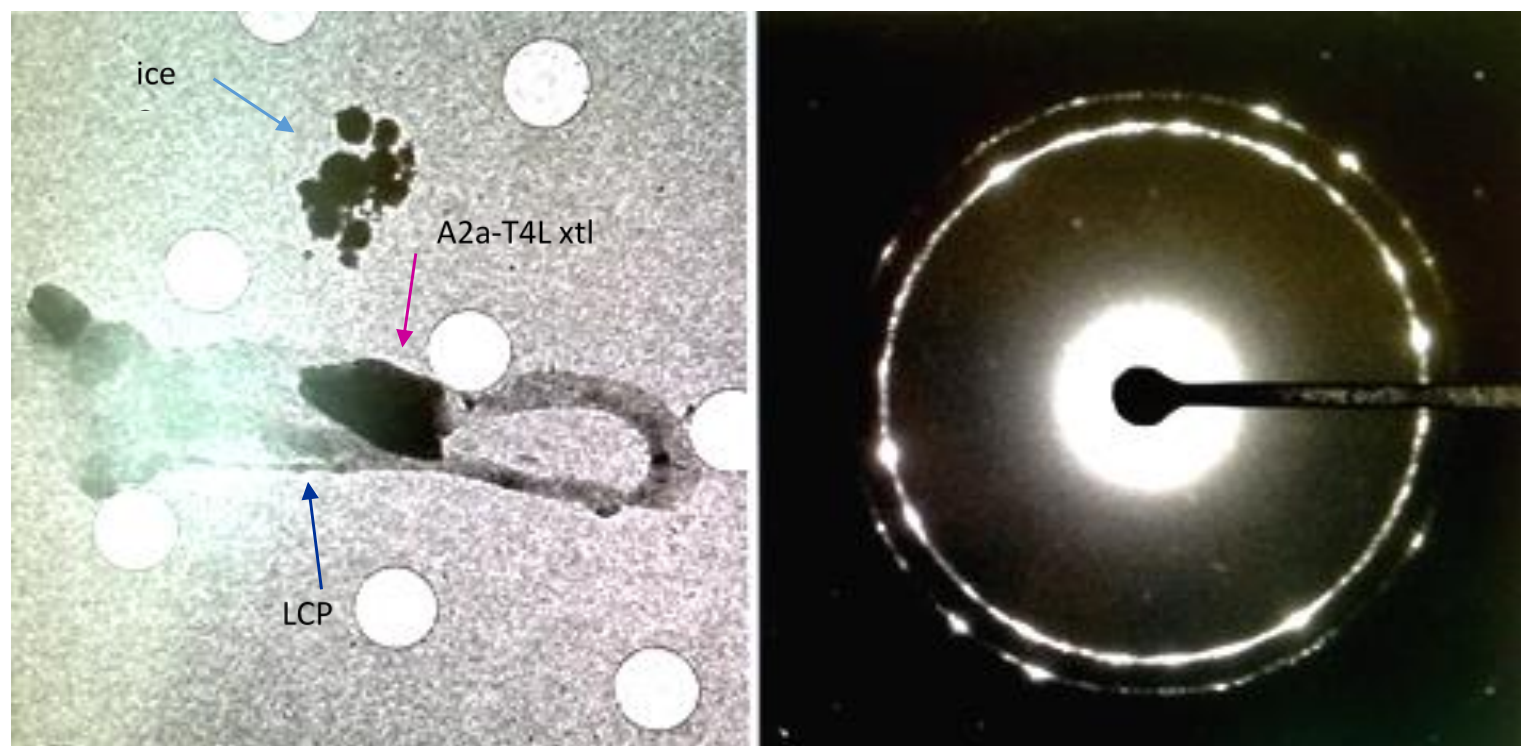

Figure 4.4. MicroED of a cryopreserved 3D $A_{2 A} A R-T 4 L$ crystal grown in LCP. Left, anvil-shaped $A_{2 A} A R-T 4 L$ crystal partially embedded in LCP over holey carbon grid. Ice also present. Right, direct diffraction of crystal in left panel showing ice rings, strong reflections arising from arising from salt diffraction (corners) and weaker reflections arising from diffraction of the $A_{2 A} A R-T 4 L$ crystal. LCP crystals from Michael Purdy, Yeager Lab. Crystal harvesting by J. Wingard, and freezing by J. Wingard and Brent Nannenga, Gonen Lab. Microscopy by Dan Shi, Gonen Lab. 


\section{Diffraction of bicelle crystals}

Bicelle crystals of the bacterial cellulose synthase complex BcsA-BcsB were applied to grids and cryopreserved as mentioned. Crystals were difficult to observe in ice due to low contrast and appeared in two forms, large plates, and ribbon-like crystals particularly sensitive to radiation damage. Figure $\mathbf{4 . 5}$ shows a representative micrograph of both crystal forms. Only the plate-like crystals were indexable in 2DX due to the radiation-sensitivity of the ribbon-like crystals. The partial unit cell dimensions of the plate-like crystals is $187 \AA$ x $405 \AA$, compared to the published dimensions, $103 \AA$ x 103 $\AA$ x $468 \AA$ for a similar 3D crystal of the same complex. The 3D crystals used in this screening were optimized using additives which may have altered crystal packing, and a 3D X-ray structure is not yet available.
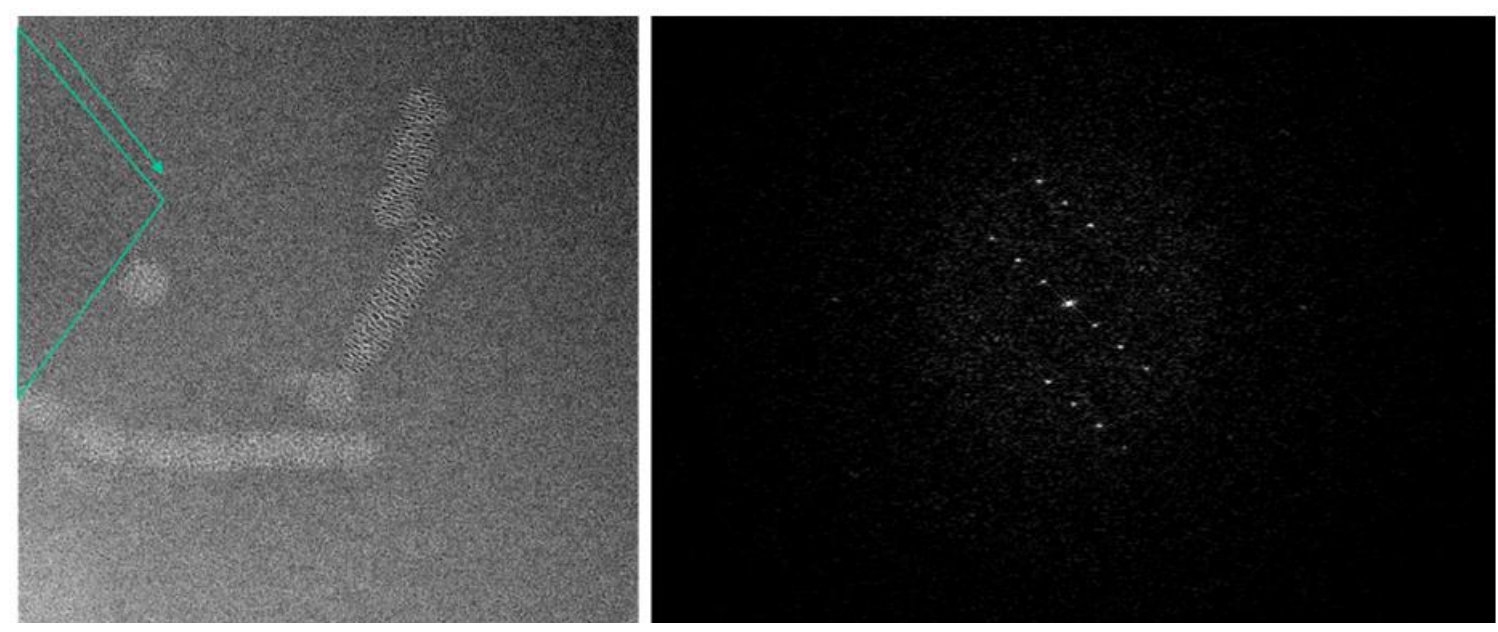

Figure 4.5. CryoEM of 3D BcsA-BcsB complex bicelle crystals and computed diffraction. Left, two crystal forms: a plate like crystal (cyan) and ribbon like crystals. Right, strong reflections from Fourier transform of masked area on left. 


\section{Discussion}

Multiple issues were encountered during the preliminary crystal screening. To an extent, the LCP served as a barrier to dehydration and freezing-induced damage. Where the LCP did not completely embed the crystals, they were frequently distorted and "freezedried". Due to this possibility, LCP-crystals required a longer screening time to diffraction than did crystals grown by vapor diffusion. Additionally, the LCP was too thick in some areas of the grid and no crystals could be selected due to the dark image. Crystal thickness was also frequently an issue during data collection, as each sample examined frequently contained crystals too thick to be imaged. Finally, ice crystal formation was also an issue, even with careful cryopreservation at UVA as well as in our scouting experiments with Gonen lab colleagues at Janelia Farm. While we were unable to determine any structures from the data, this work lays a foundation for further studies, as we are able to show diffraction from micro-LCP-grown crystals and have developed a method for crystal transfer. Additionally, successful diffraction has been observed for the first time from bicelle as well as LCP crystals.

As the technique is novel, data processing of the lysozyme crystal diffraction by the Gonen lab necessitated the development of custom software, a modified version of MOSFLM [205]. Because of abnormalities particular to electron diffraction, many high

resolution spots were discarded; despite diffraction to $1.7 \AA$, the final achievable resolution was only $2.9 \AA$ [205]. Data processing for the technique must still be optimized if we are to achieve approach the resolutions achieved with X-ray diffraction. Improvements are being made in this regard. A collection method denoted "continuous rotation" allows for 
straightforward processing using MOSFLM [207]. By collecting the electron diffraction data in a video mode as the crystal is rotated, similar to data collection in XRD where the crystal is rotated via a goniometer, the intensities are more accurately measured during sampling of the reciprocal space [207].

Furthermore, cryopreservation of macromolecular crystals is not trivial. Crystal desiccation is an issue, and considerations must be made for crystal size, beam penetration, and cryopreservation methodology. Cryopreservation of membrane protein crystals is even more difficult, as buffer viscosity and possible phase transformation become issues that must be addressed by tailored method development.

Because of their small size, micro-crystals may be more responsive in ligandsoaking experiments. Combining this potential with rapid cryopreservation could prove the method to be a valuable tool for revealing structure-function relationships. Because crystals are being examined, as opposed to single particles, it also has the potential to reveal previously unseen relationships of functional crystallographic oligomers. Additionally, because of the low radiation dosage necessary, experts at the technique may be able to examine thin, plate-like crystals precluded from traditional XRD due to their being particularly sensitive to radiation damage. Lack of ease-of-use in data collection and processing software, insufficiently detailed protocols, and the limited availability of appropriately fast direct imaging hardware such as the TVIPS or Falcon detectors, however, currently present bottlenecks to the widespread utilization of this promising technique. 


\section{Chapter 5: Future Directions}

\section{Conclusions}

In summary, this work has contributed to the general knowledge regarding the structure of GPCR-G-protein complexes and provided insight into GPCR oligomerization. Single particle analyses of an agonist GDP-bound $\mathrm{A}_{2 \mathrm{~A}} \mathrm{AR}-\mathrm{G} \alpha_{\mathrm{s}}-\beta \gamma$ complex in negative stain suggests that $\mathrm{A}_{2 \mathrm{~A}} \mathrm{AR}-\mathrm{Gs}$ complexes exist predominantly with a 1:1 stoichiometry. Likewise, single particle analysis of purified $A_{1} A R$ and $A_{2 A} A R$ constructs suggest they also exist as monomers. The $\mathrm{A}_{2 \mathrm{~A}} \mathrm{AR}$ has been visualized as a dimer in a lipid bilayer in near-native conditions. Analysis of projection density maps of negatively stained 2D, single-layered crystals of $\mathrm{A}_{2 \mathrm{~A}} \mathrm{AR}-\mathrm{T} 4 \mathrm{~L}$, suggest that the primary dimerization interface involves $\mathrm{H} 8-\mathrm{H} 1$ interactions.

Initial electron micrographic experiments involving gold-labeling suggest that following treatment with GTP $\gamma \mathrm{S}$ and complex dissociation, the adenosine receptor dimerizes readily. 3D reconstruction of GTP $\gamma$ S-treated receptors, purified using a novel tag on an intracellular loop, in conjunction with gold-labeling experiments as performed previously, would aid in confirming that G-protein activation induces conformational changes in the receptor that prime it for dimerization.

Taken together, the results suggest a model wherein receptor dimerization may be sensitive to G-protein induced conformational changes that may allosterically affect ligand binding. As ligand binding is known to influence receptor dimerization [159], this would not be 
unreasonable; however, these changes must occur within a time course reasonable for signaling. Interestingly, Monte Carlo simulations suggested a shift occurs within 0.1s from dimer to monomer following ligand binding and rapid dimerization-mediated raft partitioning [208]. In addition, negative, orthosteric, ligand-binding cooperativity has been observed in $A_{3} A R$ homodimers [209]. Furthermore, the $\beta_{2} A R$ has been shown to be internalized as a homodimer [210]. It may be that in addition to internalization, dimerization offers another degree of cellular feedback, allowing for further signaling regulation.

The structural biologist has more tools now than ever before. Figure 5.1 conveys many of these tools. Atomic force microscopy (AFM) can resolve large structural features, as can tomography, at relatively low resolution. NMR can reveal structural dynamics at high resolution, and recent advances allow for structure determination in bicelles [211] and live cells [212], allowing for structural analysis of membrane proteins in a more native state. Two cryo-EM techniques have thus far provided high resolution structures, single particle analysis and 2D electron crystallography (including image diffraction). The most popular technique providing very high resolution structures, has been x-ray diffraction of 3D crystals. High-throughput 3D crystallization methods, including liquid handling robots, and commercially available crystallization kits have positioned this method well for general use. In addition, researchers have access to a number of beamlines, in house and at numerous synchrotron sources. 


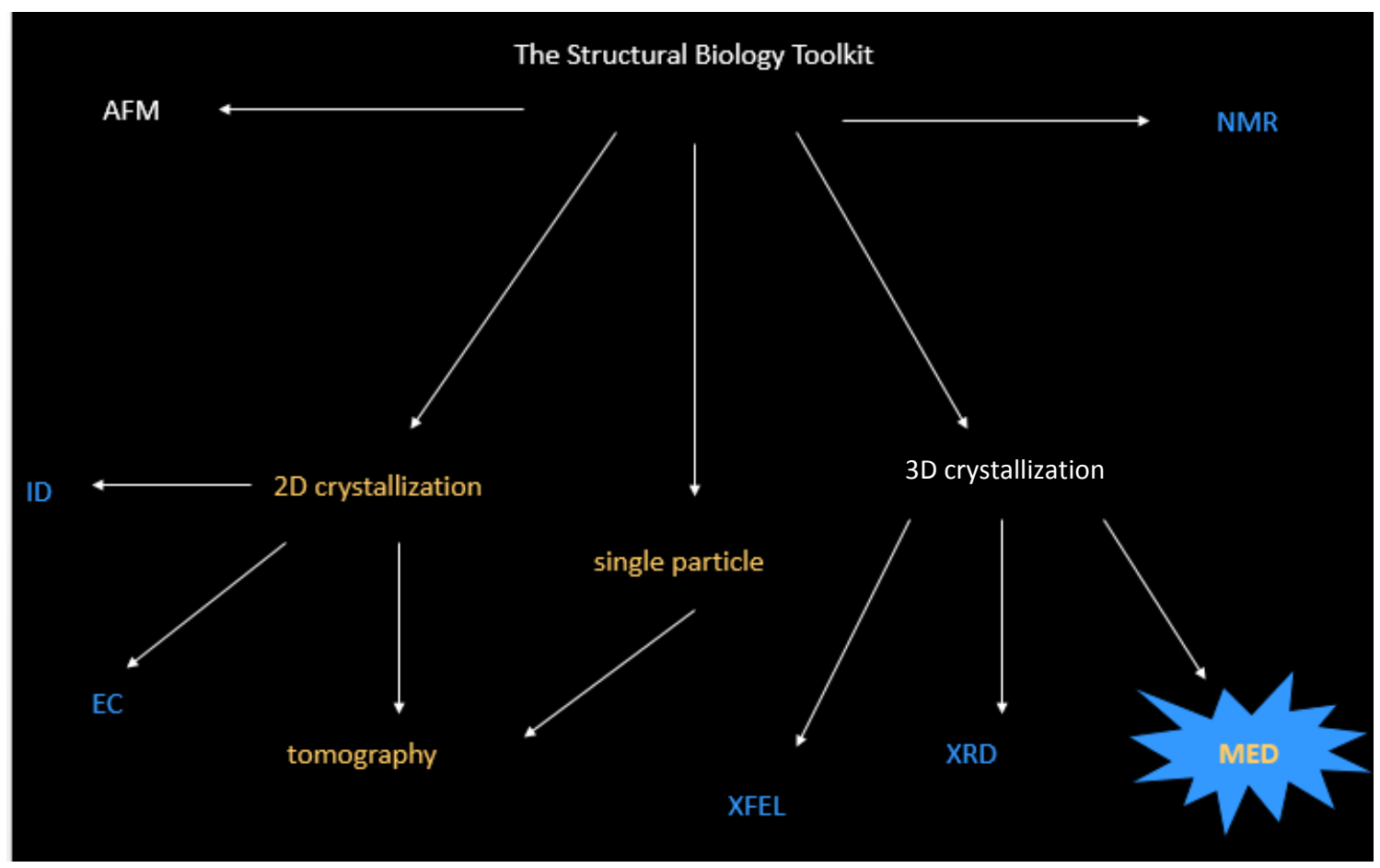

Figure 5.1. The modern structural biology toolkit. EM techniques (gold), and high-resolution methods (blue) converge in MicroED. Image diffraction (ID) from micrographs and electron crystallography $(\mathrm{EC})$ are used to generate data for processing of $2 \mathrm{D}$ crystals. Structural data from 3D crystals can be collected by x-ray diffraction (XRD), or the recently developed techniques $x$-ray free electron lasers (XFEL), or micro electron diffraction (MED). MicroED uniquely combines 3D crystallization and direct electron diffraction.

The structural biologist now has two additional tools, the x-ray free electron laser (XFEL) and MicroED. Both of the latter techniques utilize smaller than standard 3D protein crystals to generate structural data. The x-ray free electron laser utilizes short 50femotosecond pulses of $\mathrm{x}$-rays in combination with a stream of microcrystals, permitting permits millions of diffraction images per sample. Despite the high viscosity of LCP, LCPgrown crystals are amenable to the technique [213]. The short pulses are capable of generating diffraction images before radiation damage has occurred. This technique is limited however by the lack of available facilities and requires a large number of crystals. Material usage is up to $100 \mathrm{mg}$ of protein per experiment [213], a challenging quantity for the membrane protein biologist. The other recently developed technique for the structural 
biologist is MicroED. In comparison to XFEL, MicroED has the benefit of using much smaller quantities of protein. It is possible to collect a full set of diffraction date from a single 2 ul crystallization drop. Additionally, while the technique requires specialized direct electron detectors, EM facilities are much more widespread. The low electron dose allows for multiple images to be taken from the same crystal. While material usage is low, this technique is time intensive with regards to screening for 3D crystals on the surface of the EM grid. Automated data collection methods sampling diffraction from a proportion of the available grid squares have the potential of solving this issue if developed.

\section{Methods development in electron microscopy}

To a great extent, obtaining 3D crystal structures for many GPCRs has depended on LCP crystallization and in this, there has been a dependency on high-throughput methods. Unfortunately, 2D crystallization is still a very manually-intensive process, and there is no commercially available high-throughput equipment for that purpose. Obtaining the structure of a GPCR-G-protein complex by two-dimensional crystallization will be greatly aided by the development of high-throughput methods that allow for sampling and buffer exchange without experimental intervention or cessation of dialysis.

While 2D crystallization has the advantage of sampling less space than 3D crystallization, it is a thermodynamically more complex process. Ultimately, 2D crystallization occurs in lipid mixtures; carry-over of native lipids during purification, and the solubilizing lipid or combination of lipids. Each lipid has a specific melting temperature, which influences membrane fluidity, protein incorporation and movement 
within the bilayer. Because of this, 2D crystallization is likely to be sensitive to temperature fluctuations; this feature is likely to be a major obstacle to experimental repeatability. In the development of higher throughput methods for 2D crystallization, temperature control is an aspect that should not be overlooked. A deeper understanding of lipid phase transitions during crystallization is essential to evolving a brute force method into a robust scientific technique.

Another fundamental step in modernizing 2D crystallization is the development of a database suitable for analysis and data mining. The Protein Data Base (pdb.org) is a searchable database of all 3D crystal structures, and is fairly well-annotated with experimental conditions, information about the biological constructs, and complete with structures. There is likewise, a database for successful 2D crystallization experiments, compiled by the Protein Structure Initiative (http://technology.sbkb.org/portal/page/438/). Data mining of successful 2D crystallization conditions may provide some insight towards the development of improved methods, however the database is not online and as accessible as the PDB. This is another area for improvement and worthy of investment. It was cursory analysis of successful lipid mixtures used as reported in the database that provided a platform for the successful $2 \mathrm{D}$ crystallization of the adenosine receptor. A separate database, EMDB, is also available for single particle and tomographic reconstructions (http://www.ebi.ac.uk/pdbe/emdb/index.html/). While database mining has not been as fruitful for successful crystallization of 3D proteins, the lipid bilayer places a number of constraints on 2D crystals. 2D crystals typically are comprised of limited protein-protein contacts, thus given that the protein of interest is sufficiently stable in the 
buffer conditions, the lipid composition becomes paramount. Finally, expansion of EM software, 2DX, and EMAN2, into Windows compatibility would enhance accessibility, as well as bug fixes and graphical output reports for the two programs.

With the publication of the structural studies of the cytoplasmic membrane complex, $\mathrm{ExbB}_{4}-\mathrm{ExbD}_{2}$ [214], OmpF,[215] and NADH:ubiquinone oxidoreductase [216], a novel tool has come to light with potential for improving EM imaging. Amphipathic polymers or amphipols have been used to stabilize membrane proteins in the absence of detergent, and enable retention of function and ligand binding ability. The interaction of amphipols with the negative stain in EM is slightly different than detergent, and in some cases provides for higher contrast embedding. Additionally, functionalized amphipols have potential for biosensor development [217] and possibly oriented binding, as on a grid surface. Particle orientation and distribution has played a significant role in threedimensional reconstructions of GPCR-G-protein complexes. The ability to modulate particle orientation will be advantageous to the observation of small flexible domains. Due to their very low critical aggregation coefficient of $0.002 \mathrm{~g} / \mathrm{L}$ [218], they also show promise for eliminating some of the detergent artifacts seen in EM of single particles.

What is likely to make the most significant difference in the improvement of single particle data in the near future is the development of the direct detector for image capture. Challenges to collecting optimal data by EM include contrast loss during imaging due to beam-induced particle movement and inefficiency during detection by CCD coupled scintillation devices, which rely on the conversion of electrons to photons for detection. 
Direct detectors, as the name suggests, allows for direct detection of electrons via complementary metal-oxide semiconductor (CMOS) circuits with less energy loss due to heat and a fast read-out rate. Previous attempts at single particle analysis of the 4MDa 80S ribosome using 1.4 million particles resulted in a $6 \AA$ reconstruction by cryo-EM using conventional CCD detectors for imaging [219]. The most recent reconstruction calculated at $4.5 \AA$ A using only 30,000 particles, utilized a Falcon direct detector with movies allowing for correction due to beam-induced particle movement [220]. In addition to a reduction in the number of particles necessary, the improved resolution allowed the authors to resolve different conformations of the $80 \mathrm{~S}$ ribosome.

While the single particle analyses included in this work appear to confirm flexibility between $\mathrm{G} \alpha_{\text {GTPase }}$ and $\mathrm{G} \alpha_{\mathrm{H}}$ domains, higher resolution studies will be needed to follow the more subtle conformational changes that take place during receptor binding to the Gprotein. The Yeager lab now has access to a Falcon direct electron detector on the FEI Titan Krios; combined with the ability to perform automated data collection, and a sufficiently stabilized homogenous sample, a 3D reconstruction of an adenosine receptorG-protein complex at near atomic resolution should be achievable.

\section{Further Questions}

The results of this prompt a number of new questions: what conformational changes occur in the receptor occur during dimerization and how do they modulate G-protein signaling? Additional questions exist that are beyond the scope of this work to answer. Questions remain as to whether all GPCRs have the capacity for functional 
oligomerization, and if so, under what conditions. Additionally, is there a shared primary dimerization interface, or does dimerization yield different functionality depending on which of the two predominant interfaces is used? What modulates interface selection? Does the active dimer bind one or two G-proteins? Answering these questions will be challenging, biochemically. It will be necessary to produce homogenous stabilized dimers for crystallization studies and methods must be developed to exclude any head to tail dimers. High-resolution XRD studies will be necessary to follow the conformational changes that take place during dimerization. Exposure of the receptor to G-proteins and ligand selection will also be factors that need to be accounted for. Functional studies will require mutagenesis, careful ligand selection and a deeper understanding of the relationship between dimerization and cholesterol. Finally, this information must be placed into the context of a lipid bilayer in order to understand the influence of the native lipid environment on the physiological orientation of the helices. Methods development and a more technical approach to two-dimensional crystallization will be essential for success. 


\section{References}

1. Ferre, S., C. Quiroz, A.S. Woods, R. Cunha, P. Popoli, F. Ciruela, C. Lluis, R. Franco, K. Azdad, and S.N. Schiffmann, An update on adenosine A2A-dopamine D2 receptor interactions: implications for the function of $G$ protein-coupled receptors. Curr Pharm Des, 2008. 14(15): p. 1468-74.

2. Link, A.A., T. Kino, J.A. Worth, J.L. McGuire, M.L. Crane, G.P. Chrousos, R.L. Wilder, and I.J. Elenkov, Ligand-activation of the adenosine A2a receptors inhibits IL-12 production by human monocytes. J Immunol, 2000. 164(1): p. 436-42.

3. Schertler, G.F., C. Villa, and R. Henderson, Projection structure of rhodopsin. Nature, 1993. 362(6422): p. 770-2.

4. Fotiadis, D., Y. Liang, S. Filipek, D.A. Saperstein, A. Engel, and K. Palczewski, Atomic-force microscopy: rhodopsin dimers in native disc membranes. Nature, 2003. 421(6919): $p$. 127-8.

5. Jastrzebska, B., P. Ringler, D.T. Lodowski, V. Moiseenkova-Bell, M. Golczak, S.A. Muller, K. Palczewski, and A. Engel, Rhodopsin-transducin heteropentamer: three-dimensional structure and biochemical characterization. J Struct Biol, 2011. 176(3): p. 387-94.

6. Rasmussen, S., B. DeVree, Y. Zou, A. Kruse, K. Chung, T. Kobilka, F. Thian, P. Chae, E. Pardon, D. Calinski, J. Mathiesen, S. Shah, J. Lyons, M. Caffrey, S. Gellman, J. Steyaert, G. Skiniotis, W. Weis, R. Sunahara, and B. Kobilka, Crystal Structure of the B2Adrenergic Receptor-Gs protein complex. Nature, 2011 July 19. 477(7366): p. 549-555.

7. Westfield, G., Rasmussen SG, Su M, Dutta S, DeVree BT, Chung KY, Calinski D, Velez-Ruiz G, Oleskie AN, Pardon E, Chae PS, Liu T, Li S, Woods VL, Steyaert J, Kobilka BK, Sunahara RK, and S. G., Structural flexibility of the G alpha s alpha-helical domain in the beta2adrenoceptor Gs complex. Proc Natl Acad Sci U S A. , 2011 Sep 20. 108(38): p. 16086-91.

8. Scheerer, P., J.H. Park, P.W. Hildebrand, Y.J. Kim, N. Krauss, H.W. Choe, K.P. Hofmann, and O.P. Ernst, Crystal structure of opsin in its G-protein-interacting conformation. Nature, 2008. 455(7212): p. 497-502.

9. Cherezov, V., D.M. Rosenbaum, M.A. Hanson, S.G. Rasmussen, F.S. Thian, T.S. Kobilka, H.J. Choi, P. Kuhn, W.I. Weis, B.K. Kobilka, and R.C. Stevens, High-resolution crystal structure of an engineered human beta2-adrenergic $G$ protein-coupled receptor. Science, 2007. 318(5854): p. 1258-65.

10. Jafurulla, M., S. Tiwari, and A. Chattopadhyay, Identification of cholesterol recognition amino acid consensus (CRAC) motif in G-protein coupled receptors. Biochem Biophys Res Commun, 2011. 404(1): p. 569-73.

11. Hanson, M.A., V. Cherezov, M.T. Griffith, C.B. Roth, V.P. Jaakola, E.Y. Chien, J. Velasquez, P. Kuhn, and R.C. Stevens, A specific cholesterol binding site is established by the $2.8 \mathrm{~A}$ structure of the human beta2-adrenergic receptor. Structure, 2008. 16(6): p. 897-905.

12. Zheng, H., E.A. Pearsall, D.P. Hurst, Y. Zhang, J. Chu, Y. Zhou, P.H. Reggio, H.H. Loh, and P.Y. Law, Palmitoylation and membrane cholesterol stabilize mu-opioid receptor homodimerization and $G$ protein coupling. BMC Cell Biol, 2012. 13: p. 6.

13. Wang, D., X. Sun, L.M. Bohn, and W. Sadee, Opioid receptor homo- and heterodimerization in living cells by quantitative bioluminescence resonance energy transfer. Mol Pharmacol, 2005. 67(6): p. 2173-84.

14. Wall, M.A., D.E. Coleman, E. Lee, J.A. Iniguez-Lluhi, B.A. Posner, A.G. Gilman, and S.R. Sprang, The structure of the $G$ protein heterotrimer Gi alpha 1 beta 1 gamma 2. Cell, 1995. 83(6): p. 1047-58. 
15. Lambright, D.G., J. Sondek, A. Bohm, N.P. Skiba, H.E. Hamm, and P.B. Sigler, The $2.0 \mathrm{~A}$ crystal structure of a heterotrimeric $G$ protein. Nature, 1996. 379(6563): p. 311-9.

16. Rasmussen, S.G., B.T. DeVree, Y. Zou, A.C. Kruse, K.Y. Chung, T.S. Kobilka, F.S. Thian, P.S. Chae, E. Pardon, D. Calinski, J.M. Mathiesen, S.T. Shah, J.A. Lyons, M. Caffrey, S.H. Gellman, J. Steyaert, G. Skiniotis, W.I. Weis, R.K. Sunahara, and B.K. Kobilka, Crystal structure of the beta2 adrenergic receptor-Gs protein complex. Nature, 2011. 477(7366): p. 549-55.

17. Lazzerini, P.E., M. Natale, E. Gianchecchi, P.L. Capecchi, C. Montilli, S. Zimbone, M. Castrichini, E. Balistreri, G. Ricci, E. Selvi, E. Garcia-Gonzalez, M. Galeazzi, and F. LaghiPasini, Adenosine A2A receptor activation stimulates collagen production in sclerodermic dermal fibroblasts either directly and through a cross-talk with the cannabinoid system. J Mol Med (Berl), 2012. 90(3): p. 331-42.

18. Lee, C.F. and Y. Chern, Adenosine receptors and Huntington's disease. Int Rev Neurobiol, 2014. 119: p. 195-232.

19. Dammen, R., M. Haugen, B. Svejda, D. Alaimo, O. Brenna, R. Pfragner, B.I. Gustafsson, and $\mathrm{M}$. Kidd, The stimulatory adenosine receptor ADORA2B regulates serotonin (5-HT) synthesis and release in oxygen-depleted EC cells in inflammatory bowel disease. PLoS One, 2013. 8(4): p. e62607.

20. Tang, J., X. Jiang, Y. Zhou, B. Xia, and Y. Dai, Increased adenosine levels contribute to ischemic kidney fibrosis in the unilateral ureteral obstruction model. Exp Ther Med, 2015. 9(3): p. 737-743.

21. Cicala, C. and A. lalenti, Adenosine signaling in airways: toward a promising antiasthmatic approach. Eur J Pharmacol, 2013. 714(1-3): p. 522-5.

22. Chalmers, D.T. and D.P. Behan, The use of constitutively active GPCRs in drug discovery and functional genomics. Nat Rev Drug Discov, 2002. 1(8): p. 599-608.

23. Foord, S.M., T.I. Bonner, R.R. Neubig, E.M. Rosser, J.P. Pin, A.P. Davenport, M. Spedding, and A.J. Harmar, International Union of Pharmacology. XLVI. G protein-coupled receptor list. Pharmacol Rev, 2005. 57(2): p. 279-88.

24. Zhukovsky, E.A., P.R. Robinson, and D.D. Oprian, Transducin activation by rhodopsin without a covalent bond to the 11-cis-retinal chromophore. Science, 1991. 251(4993): p. 558-60.

25. Rosenbaum, D.M., V. Cherezov, M.A. Hanson, S.G. Rasmussen, F.S. Thian, T.S. Kobilka, H.J. Choi, X.J. Yao, W.I. Weis, R.C. Stevens, and B.K. Kobilka, GPCR engineering yields high-resolution structural insights into beta2-adrenergic receptor function. Science, 2007. 318(5854): p. 1266-73.

26. Shonberg, J., R.C. Kling, P. Gmeiner, and S. Lober, GPCR crystal structures: Medicinal chemistry in the pocket. Bioorg Med Chem, 2014.

27. Warne, T., M.J. Serrano-Vega, J.G. Baker, R. Moukhametzianov, P.C. Edwards, R. Henderson, A.G. Leslie, C.G. Tate, and G.F. Schertler, Structure of a beta1-adrenergic Gprotein-coupled receptor. Nature, 2008. 454(7203): p. 486-91.

28. Rasmussen, S.G., H.J. Choi, D.M. Rosenbaum, T.S. Kobilka, F.S. Thian, P.C. Edwards, M. Burghammer, V.R. Ratnala, R. Sanishvili, R.F. Fischetti, G.F. Schertler, W.I. Weis, and B.K. Kobilka, Crystal structure of the human beta2 adrenergic G-protein-coupled receptor. Nature, 2007. 450(7168): p. 383-7.

29. Warne, T., R. Moukhametzianov, J.G. Baker, R. Nehme, P.C. Edwards, A.G. Leslie, G.F. Schertler, and C.G. Tate, The structural basis for agonist and partial agonist action on a beta(1)-adrenergic receptor. Nature, 2011. 469(7329): p. 241-4. 
30. Michino, M., E. Abola, G.D. participants, C.L. Brooks, 3rd, J.S. Dixon, J. Moult, and R.C. Stevens, Community-wide assessment of GPCR structure modelling and ligand docking: GPCR Dock 2008. Nat Rev Drug Discov, 2009. 8(6): p. 455-63.

31. Jaakola, V.P., M.T. Griffith, M.A. Hanson, V. Cherezov, E.Y. Chien, J.R. Lane, A.P. ljzerman, and R.C. Stevens, The 2.6 angstrom crystal structure of a human A2A adenosine receptor bound to an antagonist. Science, 2008. 322(5905): p. 1211-7.

32. Hollenstein, K., J. Kean, A. Bortolato, R.K. Cheng, A.S. Dore, A. Jazayeri, R.M. Cooke, M. Weir, and F.H. Marshall, Structure of class B GPCR corticotropin-releasing factor receptor 1. Nature, 2013. 499(7459): p. 438-43.

33. Dore, A.S., K. Okrasa, J.C. Patel, M. Serrano-Vega, K. Bennett, R.M. Cooke, J.C. Errey, A. Jazayeri, S. Khan, B. Tehan, M. Weir, G.R. Wiggin, and F.H. Marshall, Structure of class C GPCR metabotropic glutamate receptor 5 transmembrane domain. Nature, 2014. 511(7511): p. 557-62.

34. Wang, C., H. Wu, V. Katritch, G.W. Han, X.P. Huang, W. Liu, F.Y. Siu, B.L. Roth, V. Cherezov, and R.C. Stevens, Structure of the human smoothened receptor bound to an antitumour agent. Nature, 2013. 497(7449): p. 338-43.

35. McIntire, W.E., Structural determinants involved in the formation and activation of $G$ protein betagamma dimers. Neurosignals, 2009. 17(1): p. 82-99.

36. Park, H.M., J.H. Lee, J. Yaoyao, H.J. Jun, and S.J. Lee, Limonene, a natural cyclic terpene, is an agonistic ligand for adenosine $A(2 A)$ receptors. Biochem Biophys Res Commun, 2011. 404(1): p. 345-8.

37. Feoktistov, I. and I. Biaggioni, Adenosine A2b receptors evoke interleukin-8 secretion in human mast cells. An enprofylline-sensitive mechanism with implications for asthma. J Clin Invest, 1995. 96(4): p. 1979-86.

38. Ryzhov, S., R. Zaynagetdinov, A.E. Goldstein, S.V. Novitskiy, M.R. Blackburn, I. Biaggioni, and I. Feoktistov, Effect of $A 2 B$ adenosine receptor gene ablation on adenosinedependent regulation of proinflammatory cytokines. J Pharmacol Exp Ther, 2008. 324(2): p. 694-700.

39. McColl, S.R., M. St-Onge, A.A. Dussault, C. Laflamme, L. Bouchard, J. Boulanger, and M. Pouliot, Immunomodulatory impact of the A2A adenosine receptor on the profile of chemokines produced by neutrophils. FASEB J, 2006. 20(1): p. 187-9.

40. Naganuma, M., E.B. Wiznerowicz, C.M. Lappas, J. Linden, M.T. Worthington, and P.B. Ernst, Cutting edge: Critical role for A2A adenosine receptors in the $T$ cell-mediated regulation of colitis. J Immunol, 2006. 177(5): p. 2765-9.

41. Robin, E., F. Marcillac, and E. Raddatz, A hypoxic episode during cardiogenesis downregulates the adenosinergic system and alters the myocardial anoxic tolerance. Am J Physiol Regul Integr Comp Physiol, 2015: p. ajpregu 004232014.

42. von Versen-Hoynck, F., A. Rajakumar, S.A. Bainbridge, M.J. Gallaher, J.M. Roberts, and R.W. Powers, Human placental adenosine receptor expression is elevated in preeclampsia and hypoxia increases expression of the A2A receptor. Placenta, 2009. 30(5): p. 434-42.

43. Roberts, V., B. Lu, K.M. Dwyer, and P.J. Cowan, Adenosine receptor expression in the development of renal fibrosis following ischemic injury. Transplant Proc, 2014. 46(10): p. 3257-61.

44. Feoktistov, I., S. Ryzhov, H. Zhong, A.E. Goldstein, A. Matafonov, D. Zeng, and I. Biaggioni, Hypoxia modulates adenosine receptors in human endothelial and smooth muscle cells toward an A2B angiogenic phenotype. Hypertension, 2004. 44(5): p. 649-54. 
45. Calon, F., M. Dridi, O. Hornykiewicz, P.J. Bedard, A.H. Rajput, and T. Di Paolo, Increased adenosine A2A receptors in the brain of Parkinson's disease patients with dyskinesias. Brain, 2004. 127(Pt 5): p. 1075-84.

46. Li, B., P.S. Rosenbaum, N.M. Jennings, K.M. Maxwell, and S. Roth, Differing roles of adenosine receptor subtypes in retinal ischemia-reperfusion injury in the rat. Exp Eye Res, 1999. 68(1): p. 9-17.

47. Deussen, A. and J. Schrader, Cardiac adenosine production is linked to myocardial pO2. J Mol Cell Cardiol, 1991. 23(4): p. 495-504.

48. Jonzon, B., J. Nilsson, and B.B. Fredholm, Adenosine receptor-mediated changes in cyclic AMP production and DNA synthesis in cultured arterial smooth muscle cells. J Cell Physiol, 1985. 124(3): p. 451-6.

49. St Hilaire, C., S.H. Carroll, H. Chen, and K. Ravid, Mechanisms of induction of adenosine receptor genes and its functional significance. J Cell Physiol, 2009. 218(1): p. 35-44.

50. Karmazyn, M. and M.A. Cook, Adenosine A1 receptor activation attenuates cardiac injury produced by hydrogen peroxide. Circ Res, 1992. 71(5): p. 1101-10.

51. Prabhakar, N.R. and G.K. Kumar, Oxidative stress in the systemic and cellular responses to intermittent hypoxia. Biol Chem, 2004. 385(3-4): p. 217-21.

52. Hammond, L.C., C. Bonnet, P.J. Kemp, M.S. Yates, and C.J. Bowmer, Chronic hypoxia upregulates expression of adenosine A1 receptors in DDT1-MF2 cells. Biochem Pharmacol, 2004. 67(3): p. 421-6.

53. Ben-Ari, Z., O. Pappo, J. Sulkes, Y. Cheporko, B.A. Vidne, and E. Hochhauser, Effect of adenosine $A 2 A$ receptor agonist (CGS) on ischemia/reperfusion injury in isolated rat liver. Apoptosis, 2005. 10(5): p. 955-62.

54. Strickler, J., K.A. Jacobson, and B.T. Liang, Direct preconditioning of cultured chick ventricular myocytes. Novel functions of cardiac adenosine A2a and A3 receptors. J Clin Invest, 1996. 98(8): p. 1773-9.

55. Bruno, A., G. Costantino, G. de Fabritiis, M. Pastor, and J. Selent, Membrane-sensitive conformational states of helix 8 in the metabotropic Glu2 receptor, a class C GPCR. PLoS One, 2012. 7(8): p. e42023.

56. Ramkumar, V., M. Kwatra, J.L. Benovic, G.L. Stiles, and G.L. Stilesa, Functional consequences of $A 1$ adenosine-receptor phosphorylation by the beta-adrenergic receptor kinase. Biochim Biophys Acta, 1993. 1179(1): p. 89-97.

57. Palmer, T.M. and G.L. Stiles, Stimulation of $A(2 A)$ adenosine receptor phosphorylation by protein kinase $C$ activation: evidence for regulation by multiple protein kinase $C$ isoforms. Biochemistry, 1999. 38(45): p. 14833-42.

58. Lohse, M.J., J.L. Benovic, J. Codina, M.G. Caron, and R.J. Lefkowitz, beta-Arrestin: $a$ protein that regulates beta-adrenergic receptor function. Science, 1990. 248(4962): $\mathrm{p}$. 1547-50.

59. Kirchberg, K., T.Y. Kim, M. Moller, D. Skegro, G. Dasara Raju, J. Granzin, G. Buldt, R. Schlesinger, and U. Alexiev, Conformational dynamics of helix 8 in the GPCR rhodopsin controls arrestin activation in the desensitization process. Proc Natl Acad Sci U S A, 2011. 108(46): p. 18690-5.

60. Hanson, M.A. and R.C. Stevens, Discovery of new GPCR biology: one receptor structure at a time. Structure, 2009. 17(1): p. 8-14.

61. Huber, T., S. Menon, and T.P. Sakmar, Structural basis for ligand binding and specificity in adrenergic receptors: implications for GPCR-targeted drug discovery. Biochemistry, 2008. 47(42): p. 11013-23. 
62. Wheatley, M., D. Wootten, M.T. Conner, J. Simms, R. Kendrick, R.T. Logan, D.R. Poyner, and J. Barwell, Lifting the lid on GPCRs: the role of extracellular loops. Br J Pharmacol, 2012. 165(6): p. 1688-703.

63. Granier, S. and B. Kobilka, A new era of GPCR structural and chemical biology. Nat Chem Biol, 2012. 8(8): p. 670-3.

64. Xu, F., H. Wu, V. Katritch, G.W. Han, K.A. Jacobson, Z.G. Gao, V. Cherezov, and R.C. Stevens, Structure of an agonist-bound human A2A adenosine receptor. Science, 2011. 332(6027): p. 322-7.

65. Sprang, S.R., Structural biology: a receptor unlocked. Nature, 2007. 450(7168): p. 355-6.

66. Dror, R.O., D.H. Arlow, D.W. Borhani, M.O. Jensen, S. Piana, and D.E. Shaw, Identification of two distinct inactive conformations of the beta2-adrenergic receptor reconciles structural and biochemical observations. Proc Natl Acad Sci U S A, 2009. 106(12): p. 4689-94.

67. Vogel, R., S. Martell, M. Mahalingam, M. Engelhard, and F. Siebert, Interaction of a $G$ protein-coupled receptor with a $G$ protein-derived peptide induces structural changes in both peptide and receptor: a Fourier-transform infrared study using isotopically labeled peptides. J Mol Biol, 2007. 366(5): p. 1580-8.

68. Xiang, Y., V.O. Rybin, S.F. Steinberg, and B. Kobilka, Caveolar localization dictates physiologic signaling of beta 2-adrenoceptors in neonatal cardiac myocytes. J Biol Chem, 2002. 277(37): p. 34280-6.

69. Levitt, E.S., M.J. Clark, P.M. Jenkins, J.R. Martens, and J.R. Traynor, Differential effect of membrane cholesterol removal on mu- and delta-opioid receptors: a parallel comparison of acute and chronic signaling to adenylyl cyclase. J Biol Chem, 2009. 284(33): p. 2210822.

70. Yu, P., V.A. Villar, and P.A. Jose, Methods for the study of dopamine receptors within lipid rafts of kidney cells. Methods Mol Biol, 2013. 964: p. 15-24.

71. Reversi, A., V. Rimoldi, S. Brambillasca, and B. Chini, Effects of cholesterol manipulation on the signaling of the human oxytocin receptor. Am J Physiol Regul Integr Comp Physiol, 2006. 291(4): p. R861-9.

72. Wang, D., W. Wang, Y. Duan, Y. Sun, Y. Wang, and P. Huang, Functional coupling of Gs and CFTR is independent of their association with lipid rafts in epithelial cells. Pflugers Arch, 2008. 456(5): p. 929-38.

73. Pontier, S.M., Y. Percherancier, S. Galandrin, A. Breit, C. Gales, and M. Bouvier, Cholesterol-dependent separation of the beta2-adrenergic receptor from its partners determines signaling efficacy: insight into nanoscale organization of signal transduction. J Biol Chem, 2008. 283(36): p. 24659-72.

74. Keuerleber, S., P. Thurner, C.W. Gruber, J. Zezula, and M. Freissmuth, Reengineering the collision coupling and diffusion mode of the A2A-adenosine receptor: palmitoylation in helix 8 relieves confinement. J Biol Chem, 2012. 287(50): p. 42104-18.

75. Gao, Z., Y. Ni, G. Szabo, and J. Linden, Palmitoylation of the recombinant human A1 adenosine receptor: enhanced proteolysis of palmitoylation-deficient mutant receptors. Biochem J, 1999. 342 ( Pt 2): p. 387-95.

76. Paila, Y.D., S. Tiwari, and A. Chattopadhyay, Are specific nonannular cholesterol binding sites present in G-protein coupled receptors? Biochim Biophys Acta, 2009. 1788(2): p. 295-302.

77. Ibrahim, S., A. McCartney, N. Markosyan, and E.M. Smyth, Heterodimerization with the prostacyclin receptor triggers thromboxane receptor relocation to lipid rafts. Arterioscler Thromb Vasc Biol, 2013. 33(1): p. 60-6. 
78. Hurowitz, E.H., J.M. Melnyk, Y.J. Chen, H. Kouros-Mehr, M.I. Simon, and H. Shizuya, Genomic characterization of the human heterotrimeric $G$ protein alpha, beta, and gamma subunit genes. DNA Res, 2000. 7(2): p. 111-20.

79. Michaelson, D., I. Ahearn, M. Bergo, S. Young, and M. Philips, Membrane trafficking of heterotrimeric $G$ proteins via the endoplasmic reticulum and Golgi. Mol Biol Cell, 2002. 13(9): p. 3294-302.

80. Takida, S. and P.B. Wedegaertner, Exocytic pathway-independent plasma membrane targeting of heterotrimeric G proteins. FEBS Lett, 2004. 567(2-3): p. 209-13.

81. Stow, J.L., J.B. de Almeida, N. Narula, E.J. Holtzman, L. Ercolani, and D.A. Ausiello, $A$ heterotrimeric $G$ protein, $G$ alpha $i-3$, on Golgi membranes regulates the secretion of a heparan sulfate proteoglycan in LLC-PK1 epithelial cells. J Cell Biol, 1991. 114(6): p. 1113-24.

82. Petiot, A., E. Ogier-Denis, C. Bauvy, F. Cluzeaud, A. Vandewalle, and P. Codogno, Subcellular localization of the Galphai3 protein and $G$ alpha interacting protein, two proteins involved in the control of macroautophagy in human colon cancer HT-29 cells. Biochem J, 1999. 337 ( Pt 2): p. 289-95.

83. Willard, F.S. and M.F. Crouch, Nuclear and cytoskeletal translocation and localization of heterotrimeric G-proteins. Immunol Cell Biol, 2000. 78(4): p. 387-94.

84. Ikeda, S.R., Voltage-dependent modulation of $N$-type calcium channels by G-protein beta gamma subunits. Nature, 1996. 380(6571): p. 255-8.

85. Logothetis, D.E., Y. Kurachi, J. Galper, E.J. Neer, and D.E. Clapham, The beta gamma subunits of GTP-binding proteins activate the muscarinic K+ channel in heart. Nature, 1987. 325(6102): p. 321-6.

86. Raveh, A., A. Cooper, L. Guy-David, and E. Reuveny, Nonenzymatic rapid control of GIRK channel function by a $G$ protein-coupled receptor kinase. Cell, 2010. 143(5): p. 750-60.

87. Tsunoda, Y. and C. Owyang, A newly cloned phospholipase A2-activating protein elicits $\mathrm{Ca} 2+$ oscillations and pancreatic amylase secretion via mediation of $G$ protein beta/phospholipase A2/arachidonic acid cascades. Biochem Biophys Res Commun, 1994. 203(3): p. 1716-24.

88. Friedman, E.J., B.R. Temple, S.N. Hicks, J. Sondek, C.D. Jones, and A.M. Jones, Prediction of protein-protein interfaces on G-protein beta subunits reveals a novel phospholipase $C$ beta2 binding domain. J Mol Biol, 2009. 392(4): p. 1044-54.

89. Poon, L.S., A.S. Chan, and Y.H. Wong, Gbeta3 forms distinct dimers with specific Ggamma subunits and preferentially activates the beta3 isoform of phospholipase C. Cell Signal, 2009. 21(5): p. 737-44.

90. Chidiac, P. and E.M. Ross, Phospholipase C-beta1 directly accelerates GTP hydrolysis by Galphaq and acceleration is inhibited by Gbeta gamma subunits. J Biol Chem, 1999. 274(28): p. 19639-43.

91. Chen, J., H. Ni, J. Sun, and G. Weng, G protein beta(1)gamma (2) subunits purification and their interaction with adenylyl cyclase. Sci China C Life Sci, 2003. 46(2): p. 212-23.

92. Xie, K., I. Masuho, C. Brand, C.W. Dessauer, and K.A. Martemyanov, The complex of G protein regulator RGS9-2 and Gbeta(5) controls sensitization and signaling kinetics of type 5 adenylyl cyclase in the striatum. Sci Signal, 2012. 5(239): p. ra63.

93. Zalduegui, A., M. Lopez de Jesus, L.F. Callado, J.J. Meana, and J. Salles, Levels of Gsalpha(short and long), Galpha(olf) and Gbeta(common) subunits, and calciumsensitive adenylyl cyclase isoforms $(1,5 / 6,8)$ in post-mortem human brain caudate and cortical membranes: comparison with rat brain membranes and potential stoichiometric relationships. Neurochem Int, 2011. 58(2): p. 180-9. 
94. Lenhard, J.M., M.I. Colombo, and P.D. Stahl, Heterotrimeric GTP-binding proteins (G proteins) and ADP-ribosylation factor (ARF) regulate priming of endosomal membranes for fusion. Arch Biochem Biophys, 1994. 312(2): p. 474-9.

95. Wells, C.A., Z. Zurawski, K.M. Betke, Y.Y. Yim, K. Hyde, S. Rodriguez, S. Alford, and H.E. Hamm, Gbetagamma inhibits exocytosis via interaction with critical residues on soluble $\mathrm{N}$-ethylmaleimide-sensitive factor attachment protein-25. Mol Pharmacol, 2012. 82(6): p. 1136-49.

96. Dorsam, R.T. and J.S. Gutkind, G-protein-coupled receptors and cancer. Nat Rev Cancer, 2007. 7(2): p. 79-94.

97. Vilardaga, J.P., V.O. Nikolaev, K. Lorenz, S. Ferrandon, Z. Zhuang, and M.J. Lohse, Conformational cross-talk between alpha2A-adrenergic and mu-opioid receptors controls cell signaling. Nat Chem Biol, 2008. 4(2): p. 126-31.

98. Downes, G.B. and N. Gautam, The G protein subunit gene families. Genomics, 1999. 62(3): p. 544-52.

99. Oldham, W.M. and H.E. Hamm, Heterotrimeric $G$ protein activation by G-protein-coupled receptors. Nat Rev Mol Cell Biol, 2008. 9(1): p. 60-71.

100. Heydorn, A., R.J. Ward, R. Jorgensen, M.M. Rosenkilde, T.M. Frimurer, G. Milligan, and E. Kostenis, Identification of a novel site within $G$ protein alpha subunits important for specificity of receptor-G protein interaction. Mol Pharmacol, 2004. 66(2): p. 250-9.

101. Zhang, Z., T.J. Melia, F. He, C. Yuan, A. McGough, M.F. Schmid, and T.G. Wensel, How $a$ $G$ protein binds a membrane. J Biol Chem, 2004. 279(32): p. 33937-45.

102. Mixon, M.B., E. Lee, D.E. Coleman, A.M. Berghuis, A.G. Gilman, and S.R. Sprang, Tertiary and quaternary structural changes in Gi alpha 1 induced by GTP hydrolysis. Science, 1995. 270(5238): p. 954-60.

103. Coleman, D.E., E. Lee, M.B. Mixon, M.E. Linder, A.M. Berghuis, A.G. Gilman, and S.R. Sprang, Crystallization and preliminary crystallographic studies of Gi alpha 1 and mutants of Gi alpha 1 in the GTP and GDP-bound states. J Mol Biol, 1994. 238(4): p. 6304.

104. Tesmer, J.J., D.M. Berman, A.G. Gilman, and S.R. Sprang, Structure of RGS4 bound to AlF4--activated G(i alpha1): stabilization of the transition state for GTP hydrolysis. Cell, 1997. 89(2): p. 251-61.

105. Chen, C.A. and D.R. Manning, Regulation of $G$ proteins by covalent modification. Oncogene, 2001. 20(13): p. 1643-52.

106. Gallego, C., S.K. Gupta, S. Winitz, B.J. Eisfelder, and G.L. Johnson, Myristoylation of the G alpha i2 polypeptide, a $\mathrm{G}$ protein alpha subunit, is required for its signaling and transformation functions. Proc Natl Acad Sci U S A, 1992. 89(20): p. 9695-9.

107. Taussig, R., J.A. Iniguez-Lluhi, and A.G. Gilman, Inhibition of adenylyl cyclase by Gi alpha. Science, 1993. 261(5118): p. 218-21.

108. Wedegaertner, P.B., P.T. Wilson, and H.R. Bourne, Lipid modifications of trimeric $G$ proteins. J Biol Chem, 1995. 270(2): p. 503-6.

109. Garritsen, A., P.J. van Galen, and W.F. Simonds, The N-terminal coiled-coil domain of beta is essential for gamma association: a model for G-protein beta gamma subunit interaction. Proc Natl Acad Sci U S A, 1993. 90(16): p. 7706-10.

110. Sondek, J., A. Bohm, D.G. Lambright, H.E. Hamm, and P.B. Sigler, Crystal structure of a Gprotein beta gamma dimer at 2.1A resolution. Nature, 1996. 379(6563): p. 369-74.

111. Higgins, J.B. and P.J. Casey, In vitro processing of recombinant $G$ protein gamma subunits. Requirements for assembly of an active beta gamma complex. J Biol Chem, 1994. 269(12): p. 9067-73. 
112. Iniguez-Lluhi, J.A., M.I. Simon, J.D. Robishaw, and A.G. Gilman, G protein beta gamma subunits synthesized in Sf9 cells. Functional characterization and the significance of prenylation of gamma. J Biol Chem, 1992. 267(32): p. 23409-17.

113. Fukada, Y., T. Matsuda, K. Kokame, T. Takao, Y. Shimonishi, T. Akino, and T. Yoshizawa, Effects of carboxyl methylation of photoreceptor $G$ protein gamma-subunit in visual transduction. J Biol Chem, 1994. 269(7): p. 5163-70.

114. Jian, X., W.A. Clark, J. Kowalak, S.P. Markey, W.F. Simonds, and J.K. Northup, Gbetagamma affinity for bovine rhodopsin is determined by the carboxyl-terminal sequences of the gamma subunit. J Biol Chem, 2001. 276(51): p. 48518-25.

115. Myung, C.S., W.K. Lim, J.M. DeFilippo, H. Yasuda, R.R. Neubig, and J.C. Garrison, Regions in the $G$ protein gamma subunit important for interaction with receptors and effectors. Mol Pharmacol, 2006. 69(3): p. 877-87.

116. Crouthamel, M., M.M. Thiyagarajan, D.S. Evanko, and P.B. Wedegaertner, N-terminal polybasic motifs are required for plasma membrane localization of Galpha(s) and Galpha(q). Cell Signal, 2008. 20(10): p. 1900-10.

117. Herrmann, R., M. Heck, P. Henklein, C. Kleuss, K.P. Hofmann, and O.P. Ernst, Sequence of interactions in receptor-G protein coupling. J Biol Chem, 2004. 279(23): p. 24283-90.

118. liri, T., P.S. Backlund, Jr., T.L. Jones, P.B. Wedegaertner, and H.R. Bourne, Reciprocal regulation of Gs alpha by palmitate and the beta gamma subunit. Proc Natl Acad Sci U S A, 1996. 93(25): p. 14592-7.

119. Linder, M.E., C. Kleuss, and S.M. Mumby, Palmitoylation of G-protein alpha subunits. Methods Enzymol, 1995. 250: p. 314-30.

120. Van Eps, N., W.M. Oldham, H.E. Hamm, and W.L. Hubbell, Structural and dynamical changes in an alpha-subunit of a heterotrimeric $G$ protein along the activation pathway. Proc Natl Acad Sci U S A, 2006. 103(44): p. 16194-9.

121. Lambright, D.G., J.P. Noel, H.E. Hamm, and P.B. Sigler, Structural determinants for activation of the alpha-subunit of a heterotrimeric $G$ protein. Nature, 1994. 369(6482): p. 621-8.

122. Oldham, W.M., N. Van Eps, A.M. Preininger, W.L. Hubbell, and H.E. Hamm, Mechanism of the receptor-catalyzed activation of heterotrimeric $G$ proteins. Nat Struct Mol Biol, 2006. 13(9): p. 772-7.

123. Noel, J.P., H.E. Hamm, and P.B. Sigler, The 2.2 A crystal structure of transducin-alpha complexed with GTP gamma S. Nature, 1993. 366(6456): p. 654-63.

124. Oldham, W.M. and H.E. Hamm, Structural basis of function in heterotrimeric $G$ proteins. Q Rev Biophys, 2006. 39(2): p. 117-66.

125. Palczewski, K., T. Kumasaka, T. Hori, C.A. Behnke, H. Motoshima, B.A. Fox, I. Le Trong, D.C. Teller, T. Okada, R.E. Stenkamp, M. Yamamoto, and M. Miyano, Crystal structure of rhodopsin: A G protein-coupled receptor. Science, 2000. 289(5480): p. 739-45.

126. Farrens, D.L., C. Altenbach, K. Yang, W.L. Hubbell, and H.G. Khorana, Requirement of rigid-body motion of transmembrane helices for light activation of rhodopsin. Science, 1996. 274(5288): p. 768-70.

127. Sheikh, S.P., T.A. Zvyaga, O. Lichtarge, T.P. Sakmar, and H.R. Bourne, Rhodopsin activation blocked by metal-ion-binding sites linking transmembrane helices $C$ and $F$. Nature, 1996. 383(6598): p. 347-50.

128. Janz, J.M. and D.L. Farrens, Rhodopsin activation exposes a key hydrophobic binding site for the transducin alpha-subunit C terminus. J Biol Chem, 2004. 279(28): p. 29767-73. 
129. Taylor, J.M., G.G. Jacob-Mosier, R.G. Lawton, A.E. Remmers, and R.R. Neubig, Binding of an alpha 2 adrenergic receptor third intracellular loop peptide to $G$ beta and the amino terminus of G alpha. J Biol Chem, 1994. 269(44): p. 27618-24.

130. Kisselev, O., M. Ermolaeva, and N. Gautam, Efficient interaction with a receptor requires a specific type of prenyl group on the G protein gamma subunit. J Biol Chem, 1995. 270(43): p. 25356-8.

131. Oldham, W.M. and H.E. Hamm, How do receptors activate G proteins? Adv Protein Chem, 2007. 74: p. 67-93.

132. Schwindinger, W.F., A. Miric, D. Zimmerman, and M.A. Levine, A novel Gs alpha mutant in a patient with Albright hereditary osteodystrophy uncouples cell surface receptors from adenylyl cyclase. J Biol Chem, 1994. 269(41): p. 25387-91.

133. Sullivan, K.A., R.T. Miller, S.B. Masters, B. Beiderman, W. Heideman, and H.R. Bourne, Identification of receptor contact site involved in receptor- $G$ protein coupling. Nature, 1987. 330(6150): p. 758-60.

134. Onrust, R., P. Herzmark, P. Chi, P.D. Garcia, O. Lichtarge, C. Kingsley, and H.R. Bourne, Receptor and betagamma binding sites in the alpha subunit of the retinal $G$ protein transducin. Science, 1997. 275(5298): p. 381-4.

135. Ho, M.K. and Y.H. Wong, The amino terminus of Galpha(z) is required for receptor recognition, whereas its alpha4/beta6 loop is essential for inhibition of adenylyl cyclase. Mol Pharmacol, 2000. 58(5): p. 993-1000.

136. Rasenick, M.M., M. Watanabe, M.B. Lazarevic, S. Hatta, and H.E. Hamm, Synthetic peptides as probes for $G$ protein function. Carboxyl-terminal $G$ alpha s peptides mimic $G s$ and evoke high affinity agonist binding to beta-adrenergic receptors. J Biol Chem, 1994. 269(34): p. 21519-25.

137. Cai, K., Y. Itoh, and H.G. Khorana, Mapping of contact sites in complex formation between transducin and light-activated rhodopsin by covalent crosslinking: use of a photoactivatable reagent. Proc Natl Acad Sci U S A, 2001. 98(9): p. 4877-82.

138. Nanoff, C., R. Koppensteiner, Q. Yang, E. Fuerst, H. Ahorn, and M. Freissmuth, The carboxyl terminus of the Galpha-subunit is the latch for triggered activation of heterotrimeric $G$ proteins. Mol Pharmacol, 2006. 69(1): p. 397-405.

139. Hamm, H.E., A.I. Kaya, J.A. Gilbert, 3rd, and A.M. Preininger, Linking receptor activation to changes in Sw I and II of Galpha proteins. J Struct Biol, 2013. 184(1): p. 63-74.

140. Hofmann, K.P. and J. Reichert, Chemical probing of the light-induced interaction between rhodopsin and G-protein. Near-infrared light-scattering and sulfhydryl modifications. J Biol Chem, 1985. 260(13): p. 7990-5.

141. Alves, I.D., G.F. Salgado, Z. Salamon, M.F. Brown, G. Tollin, and V.J. Hruby, Phosphatidylethanolamine enhances rhodopsin photoactivation and transducin binding in a solid supported lipid bilayer as determined using plasmon-waveguide resonance spectroscopy. Biophys J, 2005. 88(1): p. 198-210.

142. Cerione, R.A., J.W. Regan, H. Nakata, J. Codina, J.L. Benovic, P. Gierschik, R.L. Somers, A.M. Spiegel, L. Birnbaumer, R.J. Lefkowitz, and et al., Functional reconstitution of the alpha 2-adrenergic receptor with guanine nucleotide regulatory proteins in phospholipid vesicles. J Biol Chem, 1986. 261(8): p. 3901-9.

143. Preininger, A.M., N. Van Eps, N.J. Yu, M. Medkova, W.L. Hubbell, and H.E. Hamm, The myristoylated amino terminus of Galpha(i)(1) plays a critical role in the structure and function of Galpha(i)(1) subunits in solution. Biochemistry, 2003. 42(26): p. 7931-41. 
144. Scheerer, P., M. Heck, A. Goede, J.H. Park, H.W. Choe, O.P. Ernst, K.P. Hofmann, and P.W. Hildebrand, Structural and kinetic modeling of an activating helix switch in the rhodopsin-transducin interface. Proc Natl Acad Sci U S A, 2009. 106(26): p. 10660-5.

145. Kobilka, B.K., $G$ protein coupled receptor structure and activation. Biochim Biophys Acta, 2007. 1768(4): p. 794-807.

146. Abdulaev, N.G., T. Ngo, C. Zhang, A. Dinh, D.M. Brabazon, K.D. Ridge, and J.P. Marino, Heterotrimeric G-protein alpha-subunit adopts a "preactivated" conformation when associated with betagamma-subunits. J Biol Chem, 2005. 280(45): p. 38071-80.

147. Oldham, W.M., N. Van Eps, A.M. Preininger, W.L. Hubbell, and H.E. Hamm, Mapping allosteric connections from the receptor to the nucleotide-binding pocket of heterotrimeric G proteins. Proc Natl Acad Sci U S A, 2007. 104(19): p. 7927-32.

148. Preininger, A.M., M.A. Funk, W.M. Oldham, S.M. Meier, C.A. Johnston, S. Adhikary, A.J. Kimple, D.P. Siderovski, H.E. Hamm, and T.M. Iverson, Helix dipole movement and conformational variability contribute to allosteric GDP release in Galphai subunits. Biochemistry, 2009. 48(12): p. 2630-42.

149. Higashijima, T., K.M. Ferguson, P.C. Sternweis, M.D. Smigel, and A.G. Gilman, Effects of $\mathrm{Mg} 2+$ and the beta gamma-subunit complex on the interactions of guanine nucleotides with $G$ proteins. J Biol Chem, 1987. 262(2): p. 762-6.

150. Abdulaev, N.G., T. Ngo, E. Ramon, D.M. Brabazon, J.P. Marino, and K.D. Ridge, The receptor-bound "empty pocket" state of the heterotrimeric G-protein alpha-subunit is conformationally dynamic. Biochemistry, 2006. 45(43): p. 12986-97.

151. Guo, W., E. Urizar, M. Kralikova, J.C. Mobarec, L. Shi, M. Filizola, and J.A. Javitch, Dopamine $D 2$ receptors form higher order oligomers at physiological expression levels. EMBO J, 2008. 27(17): p. 2293-304.

152. Zhao, Y., C. Tong, and J. Jiang, Hedgehog regulates smoothened activity by inducing a conformational switch. Nature, 2007. 450(7167): p. 252-8.

153. Monnier, C., H. Tu, E. Bourrier, C. Vol, L. Lamarque, E. Trinquet, J.P. Pin, and P. Rondard, Trans-activation between 7TM domains: implication in heterodimeric GABAB receptor activation. EMBO J, 2011. 30(1): p. 32-42.

154. Spaulding, T.C., S. Fielding, J.J. Venafro, and H. Lal, Antinociceptive activity of clonidine and its potentiation of morphine analgesia. Eur J Pharmacol, 1979. 58(1): p. 19-25.

155. AbdAlla, S., H. Lother, A. el Massiery, and U. Quitterer, Increased AT(1) receptor heterodimers in preeclampsia mediate enhanced angiotensin II responsiveness. Nat Med, 2001. 7(9): p. 1003-9.

156. Kalda, A., L. Yu, E. Oztas, and J.F. Chen, Novel neuroprotection by caffeine and adenosine $A(2 A)$ receptor antagonists in animal models of Parkinson's disease. J Neurol Sci, 2006. 248(1-2): p. 9-15.

157. Laffray, S., R. Bouali-Benazzouz, M.A. Papon, A. Favereaux, Y. Jiang, T. Holm, C. Spriet, P. Desbarats, P. Fossat, Y. Le Feuvre, M. Decossas, L. Heliot, U. Langel, F. Nagy, and M. Landry, Impairment of GABAB receptor dimer by endogenous 14-3-3zeta in chronic pain conditions. EMBO J, 2012. 31(15): p. 3239-51.

158. Periole, X., A.M. Knepp, T.P. Sakmar, S.J. Marrink, and T. Huber, Structural determinants of the supramolecular organization of $G$ protein-coupled receptors in bilayers. J Am Chem Soc, 2012. 134(26): p. 10959-65.

159. Xue, L., X. Rovira, P. Scholler, H. Zhao, J. Liu, J.P. Pin, and P. Rondard, Major ligandinduced rearrangement of the heptahelical domain interface in a GPCR dimer. Nat Chem Biol, 2015. 11(2): p. 134-40. 
160. Kim, S.K. and K.A. Jacobson, Computational prediction of homodimerization of the A3 adenosine receptor. J Mol Graph Model, 2006. 25(4): p. 549-61.

161. Liu, W., E. Chun, A.A. Thompson, P. Chubukov, F. Xu, V. Katritch, G.W. Han, C.B. Roth, L.H. Heitman, I.J. AP, V. Cherezov, and R.C. Stevens, Structural basis for allosteric regulation of GPCRs by sodium ions. Science, 2012. 337(6091): p. 232-6.

162. Lebon, G., T. Warne, P.C. Edwards, K. Bennett, C.J. Langmead, A.G. Leslie, and C.G. Tate, Agonist-bound adenosine A2A receptor structures reveal common features of GPCR activation. Nature, 2011. 474(7352): p. 521-5.

163. Hino, T., T. Arakawa, H. Iwanari, T. Yurugi-Kobayashi, C. Ikeda-Suno, Y. Nakada-Nakura, O. Kusano-Arai, S. Weyand, T. Shimamura, N. Nomura, A.D. Cameron, T. Kobayashi, T. Hamakubo, S. Iwata, and T. Murata, G-protein-coupled receptor inactivation by an allosteric inverse-agonist antibody. Nature, 2012. 482(7384): p. 237-40.

164. Bohn, S., F. Beck, E. Sakata, T. Walzthoeni, M. Beck, R. Aebersold, F. Forster, W. Baumeister, and S. Nickell, Structure of the 26S proteasome from Schizosaccharomyces pombe at subnanometer resolution. Proc Natl Acad Sci U S A, 2010. 107(49): p. 20992-7.

165. Cheng, Y. and T. Walz, The advent of near-atomic resolution in single-particle electron microscopy. Annu Rev Biochem, 2009. 78: p. 723-42.

166. Ludtke, S.J., P.R. Baldwin, and W. Chiu, EMAN: semiautomated software for highresolution single-particle reconstructions. J Struct Biol, 1999. 128(1): p. 82-97.

167. Murin, C.D., J.P. Julien, D. Sok, R.L. Stanfield, R. Khayat, A. Cupo, J.P. Moore, D.R. Burton, I.A. Wilson, and A.B. Ward, Structure of $2 \mathrm{G} 12$ Fab2 in complex with soluble and fully glycosylated HIV-1 Env by negative-stain single-particle electron microscopy. J Virol, 2014. 88(17): p. 10177-88.

168. Hu, M., L. Qian, R.P. Brinas, E.S. Lymar, L. Kuznetsova, and J.F. Hainfeld, Gold nanoparticle-protein arrays improve resolution for cryo-electron microscopy. J Struct Biol, 2008. 161(1): p. 83-91.

169. Sakmar, T.P., Biochemistry. Redder than red. Science, 2012. 338(6112): p. 1299-300.

170. Filipek, S., Organization of rhodopsin molecules in native membranes of rod cells--an old theoretical model compared to new experimental data. J Mol Model, 2005. 11(4-5): p. 385-91.

171. Jastrzebska, B., M. Golczak, D. Fotiadis, A. Engel, and K. Palczewski, Isolation and functional characterization of a stable complex between photoactivated rhodopsin and the $G$ protein, transducin. FASEB J, 2009. 23(2): p. 371-81.

172. Jastrzebska, B., P. Ringler, K. Palczewski, and A. Engel, The rhodopsin-transducin complex houses two distinct rhodopsin molecules. J Struct Biol, 2013. 182(2): p. 164-72.

173. Jastrzebska, B., T. Orban, M. Golczak, A. Engel, and K. Palczewski, Asymmetry of the rhodopsin dimer in complex with transducin. FASEB J, 2013. 27(4): p. 1572-84.

174. Ohi, M., Y. Li, Y. Cheng, and T. Walz, Negative Staining and Image Classification Powerful Tools in Modern Electron Microscopy. Biol Proced Online, 2004. 6: p. 23-34.

175. Collins, R.F., M. Saleem, and J.P. Derrick, Purification and three-dimensional electron microscopy structure of the Neisseria meningitidis type IV pilus biogenesis protein PilG. J Bacteriol, 2007. 189(17): p. 6389-96.

176. Kelly, D.F., P.D. Abeyrathne, D. Dukovski, and T. Walz, The Affinity Grid: a pre-fabricated EM grid for monolayer purification. J Mol Biol, 2008. 382(2): p. 423-33.

177. Bigler Wang, D., N.E. Sherman, J.D. Shannon, S.A. Leonhardt, L.H. Mayeenuddin, M. Yeager, and W.E. McIntire, Binding of beta4gamma5 by adenosine A1 and A2A receptors determined by stable isotope labeling with amino acids in cell culture and mass spectrometry. Biochemistry, 2011. 50(2): p. 207-20. 
178. Wettschureck, N. and S. Offermanns, Mammalian $G$ proteins and their cell type specific functions. Physiol Rev, 2005. 85(4): p. 1159-204.

179. Tucker, A.L. and J. Linden, Cloned receptors and cardiovascular responses to adenosine. Cardiovasc Res, 1993. 27(1): p. 62-7.

180. Calebiro, D., F. Rieken, J. Wagner, T. Sangkaworn, U. Zabel, A. Borzi, E. Cocucci, A. Zurn, and $\mathrm{M}$. Lohse, Single-molecule analysis of fluorescently-labeled G-protein-coupled receptors rveals complexes with distinct dynamics and organization. PNAS, 2013. 110: $\mathrm{p}$. 743-748.

181. Golebiewska, U., Johnston JM, Devi L, Filizola M, and S. S., Differential response to morphine of the oligomeric state of mu-opioid in the prescence of delta-opioid receptors. Biochemistry, 2011. 50: p. 2829-2837.

182. Hebert, T., Moffett S, Morello JP, Loisel TP, Bichet DG, B. C, and B. MA., A peptide derived from a 62 -adrenergic receptor transmembrane domain inhibits both receptor dimerization and activation. J Biol Chem, 1996. 271: p. 16384-16392.

183. Baneres, J. and J. Parello, Structure-based analysis of GPCR functional evidence for a novel pentameric assembly between the dimeric leukotriene B4 receptor BLT1 and the Gprotein. J. Mol. Biol., 2003. 329: p. 813-829.

184. Huang, J., Chen S, Zhang JJ, and H. XY., Crystal structure of oligomeric B1-adrengeric G protein coupled receptors in ligand-free basal state. Nat Struct Biol, 2013. 20: p. 419425.

185. Guo, W., L. Shi, M. Filizola, H. Weinstein, and J.A. Javitch, Crosstalk in G protein-coupled receptors: changes at the transmembrane homodimer interface determine activation. Proc Natl Acad Sci U S A, 2005. 102(48): p. 17495-500.

186. Mancia, F., Z. Assur, A.G. Herman, R. Siegel, and W.A. Hendrickson, Ligand sensitivity in dimeric associations of the serotonin 5HT2c receptor. EMBO Rep, 2008. 9(4): p. 363-9.

187. Hu, J., K. Hu, T. Liu, M.K. Stern, R. Mistry, R.A. Challiss, S. Costanzi, and J. Wess, Novel structural and functional insights into M3 muscarinic receptor dimer/oligomer formation. J Biol Chem, 2013. 288(48): p. 34777-90.

188. Ruprecht, J., ., Mielke T, Vogel R, Villa C, and S. GFX, Electron crystallography reveals the structure of metarhodopsin I. EMBO J, 2004. 23: p. 3609-3620.

189. Manglik, A., Kruse AC, Kobilka TS, Thian FS, Mathiesen JM, Sunahara RK, Pardo L, Weis $\mathrm{WI}, \mathrm{K} . \mathrm{BK}$, and G. S, Crystal structure of the mu-opioid receptor bound to a morphianan antagonist. Nature, 2012. 485: p. 321-326.

190. Wu, H., Wacker D, Mileni M, Katritch V, Han GW, Vardy E, Liu W, Thompson AA, Huang $\mathrm{XP}$, and C. FL, Structure of the human kappa-opioid receptor in complex with JDTic. Nature, 2012. 485: p. 327-332.

191. Liang, Y., Fotiadis D, Filipek S, Saperstein DA, Palczewski K, and E. A., Organization of the $G$ protein coupled receptor rhodopsin and opsin in native membranes. J Biol Chem, 2003. 278: p. 21655-21662.

192. Salom, D., Lodowski DT, Stenkamp RE, LeTrong I, Golczak M, Jastrzebska B, Harris T, Ballesteros JA, and P. K, Crystal structure of a photoactivated deprotonated intermediate of rhodopsin. PNAS, 2006. 103: p. 16123-16128.

193. Knepp, A., Periole X, Marrink SJ, Sakmar TP, and H. T., Rhodopsin forms a dimer with cytoplasmic helix 8 contacts in native membranes. Biochemistry, 2012. 51: p. 1819-1821.

194. Mielke, T., Villa C, Edwards PC, Schertler GFX, and H. MP, X-ray Diffraction of Heavyatom Labelled Two-dimensional Crystals of Rhodopsin Identifies the Position of Cysteine 140 in Helix 3 and Cysteine 316 in Helix 8. J Mol Biol, 2002. 316: p. 693-709. 
195. Rigaud, J.-L., M. Chami, O. Lambert, D. Levy, and J.-L. Ranck, Use of detergents in twodimensional crystallization of membrane proteins. Biochimica et Biophysica Acta (BBA) Biomembranes, 2000(1508): p. 112-128.

196. Zhuang, J., G.G. Prive, G.E. Werner, P. Ringler, H.R. Kaback, and A. Engel, TwoDimensional Crystallization of Escherichia coli Lactose Permease. Journal of Struct Biol, 1999. 125(1): p. 63-75.

197. Krebs, A., C. Villa, P.C. Edwards, and G.F. Schertler, Characterisation of an improved twodimensional p22121 crystal from bovine rhodopsin. J Mol Biol, 1998. 282(5): p. 9911003.

198. Davies, A., G. Schertler, B. Gowen, and H. Saibil, Projection structure of an invertebrate rhodopsin. J Struct Biol, 1996. 117: p. 36-44.

199. Unger, V. and G. Schertler, Low resolution structure of bovine rhodopsin determined by electron cryo-microscopy. Biophys J, 1995. 68: p. 1776-1786.

200. Ferre, S., V. Casado, L. Devi, M. Filizola, R. Jockers, M. Lohse, G. Milligan, J. Pin, and X. Guitart, G-protein coupled receptor oligomerization revisited: functional and pharmacological perspectives. Pharmacol Rev, 2014. 66: p. 413-434.

201. Gipson, B., X. Zeng, Z.Y. Zhang, and H. Stahlberg, 2dx--user-friendly image processing for 2D crystals. J Struct Biol, 2007. 157(1): p. 64-72.

202. Nannenga, B.L., D. Shi, J. Hattne, F.E. Reyes, and T. Gonen, Structure of catalase determined by MicroED. Elife, 2014. 3: p. e03600.

203. Nannenga, B.L. and T. Gonen, Protein structure determination by MicroED. Curr Opin Struct Biol, 2014. 27: p. 24-31.

204. Nederlof, I., E. van Genderen, Y.W. Li, and J.P. Abrahams, A Medipix quantum area detector allows rotation electron diffraction data collection from submicrometre threedimensional protein crystals. Acta Crystallogr D Biol Crystallogr, 2013. 69(Pt 7): p. 122330.

205. Shi, D., B.L. Nannenga, M.G. Iadanza, and T. Gonen, Three-dimensional electron crystallography of protein microcrystals. Elife, 2013. 2: p. e01345.

206. Morgan, J.L., J.T. McNamara, and J. Zimmer, Mechanism of activation of bacterial cellulose synthase by cyclic di-GMP. Nat Struct Mol Biol, 2014. 21(5): p. 489-96.

207. Nannenga, B.L., D. Shi, A.G. Leslie, and T. Gonen, High-resolution structure determination by continuous-rotation data collection in MicroED. Nat Methods, 2014. 11(9): p. 927-30.

208. Fallahi-Sichani, M. and J.J. Linderman, Lipid raft-mediated regulation of G-protein coupled receptor signaling by ligands which influence receptor dimerization: $a$ computational study. PLoS One, 2009. 4(8): p. e6604.

209. May, L.T., L.J. Bridge, L.A. Stoddart, S.J. Briddon, and S.J. Hill, Allosteric interactions across native adenosine-A3 receptor homodimers: quantification using single-cell ligandbinding kinetics. FASEB J, 2011. 25(10): p. 3465-76.

210. Sartania, N., S. Appelbe, J.D. Pediani, and G. Milligan, Agonist occupancy of a single monomeric element is sufficient to cause internalization of the dimeric beta2adrenoceptor. Cell Signal, 2007. 19(9): p. 1928-38.

211. Kucharska, I., T.C. Edrington, B. Liang, and L.K. Tamm, Optimizing nanodiscs and bicelles for solution NMR studies of two beta-barrel membrane proteins. J Biomol NMR, 2015. 61(3-4): p. 261-74.

212. Selenko, P. and G. Wagner, Looking into live cells with in-cell NMR spectroscopy. J Struct Biol, 2007. 158(2): p. 244-53. 
213. Nogly, P., D. James, D. Wang, T.A. White, N. Zatsepin, A. Shilova, G. Nelson, H. Liu, L. Johansson, M. Heymann, K. Jaeger, M. Metz, C. Wickstrand, W. Wu, P. Bath, P. Berntsen, D. Oberthuer, V. Panneels, V. Cherezov, H. Chapman, G. Schertler, R. Neutze, J. Spence, I. Moraes, M. Burghammer, J. Standfuss, and U. Weierstall, Lipidic cubic phase serial millisecond crystallography using synchrotron radiation. IUCrJ, 2015. 2(Pt 2): p. 168-76.

214. Sverzhinsky, A., S. Qian, L. Yang, M. Allaire, I. Moraes, D. Ma, J.W. Chung, M. Zoonens, J.L. Popot, and J.W. Coulton, Amphipol-trapped ExbB-ExbD membrane protein complex from Escherichia coli: a biochemical and structural case study. J Membr Biol, 2014. 247(9-10): p. 1005-18.

215. Arunmanee, W., J.R. Harris, and J.H. Lakey, Outer membrane protein F stabilised with minimal amphipol forms linear arrays and LPS-dependent 2D crystals. J Membr Biol, 2014. 247(9-10): p. 949-56.

216. Flotenmeyer, M., H. Weiss, C. Tribet, J.L. Popot, and K. Leonard, The use of amphipathic polymers for cryo electron microscopy of NADH:ubiquinone oxidoreductase (complex I). J Microsc, 2007. 227(Pt 3): p. 229-35.

217. Della Pia, E.A., J.V. Holm, N. Lloret, C. Le Bon, J.L. Popot, M. Zoonens, J. Nygard, and K.L. Martinez, A step closer to membrane protein multiplexed nanoarrays using biotin-doped polypyrrole. ACS Nano, 2014. 8(2): p. 1844-53.

218. Giusti, F., J.L. Popot, and C. Tribet, Well-defined critical association concentration and rapid adsorption at the air/water interface of a short amphiphilic polymer, amphipol A835: a study by Forster resonance energy transfer and dynamic surface tension measurements. Langmuir, 2012. 28(28): p. 10372-80.

219. Armache, J.P., A. Jarasch, A.M. Anger, E. Villa, T. Becker, S. Bhushan, F. Jossinet, M. Habeck, G. Dindar, S. Franckenberg, V. Marquez, T. Mielke, M. Thomm, O. Berninghausen, B. Beatrix, J. Soding, E. Westhof, D.N. Wilson, and R. Beckmann, CryoEM structure and rRNA model of a translating eukaryotic 80S ribosome at 5.5-A resolution. Proc Natl Acad Sci U S A, 2010. 107(46): p. 19748-53.

220. Bai, X.C., I.S. Fernandez, G. McMullan, and S.H. Scheres, Ribosome structures to nearatomic resolution from thirty thousand cryo-EM particles. Elife, 2013. 2: p. e00461. 


\section{Acknowledgements}

To acknowledge all that brought me here, I must include the Infinite beauty, the Infinite complexity of the Universe. I would be remiss if I did not recall the questions of my sixyear old self underneath an oak tree, who used broken stalks of bamboo for her first test tubes. Thanks to my mother, who did not seem to mind repainting a wall after my early chemistry experiments wherein I emptied the cabinet in an attempt to discover what dissolves what, and how viscosity changes when you mix two solutions together long before I knew the meaning of the word "chemistry", for two long drives from Maryland to Alfred University in New York the summer I was 15 so that I could drive a scanning electron microscope, and for years of subscriptions to Discover magazine. Thanks to my father for weekly trips to the library. Thanks to both my parents for accepting my preference for anatomy models to dolls and scopes to sports.

Having no relation to scientists early in my life, my inspirations were as mountains on the horizon, dream-worthy, guiding, but removed. I thank Mr. Asner, one of my high school biology teachers, for enthusiastic teaching, but most of all for stating his personal views on the complexity of the Universe, and keeping the two distinct. With great gratitude, I thank Mr. McDaniels, a perceptive high school English teacher who changed the course of my life with his recognition and effort. I thank two people from high school I can no longer remember the names of: a female microbiologist who challenged me by saying if I wanted to be a scientist then I should read the scientific literature, and an older gentleman who 
visited the coffee shop where I earned my tuition money and gave me my first labcoat, a labcoat that I still use today.

I am thankful for the memory of my undergraduate advisor, Dr. James Perham; his guiding anecdotes, his recognition of my strengths, integrity, and for giving me my first scientific job, as his lab tech and teaching assistant. With deep affection, I thank my college Chemistry professor, Dr. William Mattson, who has been my greatest personal inspiration. Because of him, I learned my strengths; from him, I learned what teaching is. He is my intellectual hero.

I am thankful for the technical instruction and friendship of Dr. Sean Michael Kelly, formerly at Loyola University Chicago, and graduate of OHSU. He singlehandedly taught me most of what I know about laboratory science and made the 'hood', a pleasant place to be. I am thankful for the friendship and collaboration of Dr. Jane Ladner of NIST and for the mentorship of Dr. James B. Ames, whose passion for science eclipses any that I have yet seen. I thank the amply-degreed Dr. Aswani Valiveti, Dr. Brian Kobilka, Dr. Andreas Engel and Dr. Zvi Kelman for their encouragement.

I thank my college best friend, Dr. Nileema Vaswani, for her constant friendship and support. Thank you to Dr. Joshua King who brought me with him to the University of Virginia, for his constancy and for double-checking my calculations at my request. I thank long-time friends at UVA, Dr. Virginia Carroll, Dr. Landon Locke, Dr. Eliza Zylkiewicz, Dr. Bartek Blus, and Dr. Maciej Wiktor for their support and friendship. I also thank Maciej 
Jagelnicki, a very bright fellow student, for his camaraderie, coffee, and conversation with regards to the challenges of single particle analysis. I thank Dr. Peter Horanyi for being an all-around great guy; his intelligence and humility make for an unbeatable combination. I thank Dr. William McIntire, Dr. Michael Purdy, and Dr. Susan Leonhardt for their collaboration and for seeing me through. I thank Dr. Kelly Dryden for instruction on the transmission electron microscope. Thank you to Dr. Barbie Ganser-Pornillos, Dr. Mark Daniels, Dr. Brad Bennett and Jarek Sedzicki for assistance. Much gratitude to my committee members Dr. Jim Garrison, Dr. Michael Wiener, Dr. Linda Columbus, and Dr. Zygmunt Derewenda. A special thanks and much respect to Dr. Robert Nakamoto, for wearing many hats, and wearing them all well during my time at UVA. Great thanks to my advisor, Mark Yeager, for his vision and for providing space for this work to arise. 